\title{
Preparation of Homoallylic Homopropargylic Alcohols from 2-Vinyloxiranes
}

Spectra

Matthew L. Maddess, Mark Lautens.*

80 St. George Street, Davenport Research Laboratories, Department of Chemistry, University of Toronto, Toronto, Ontario, M5S 3H6, Canada

mlautens@chem.utoronto.ca 
(2): (+/-)-(1E)-2-Methyl-1-phenylhex-1-en-5-yn-3-ol

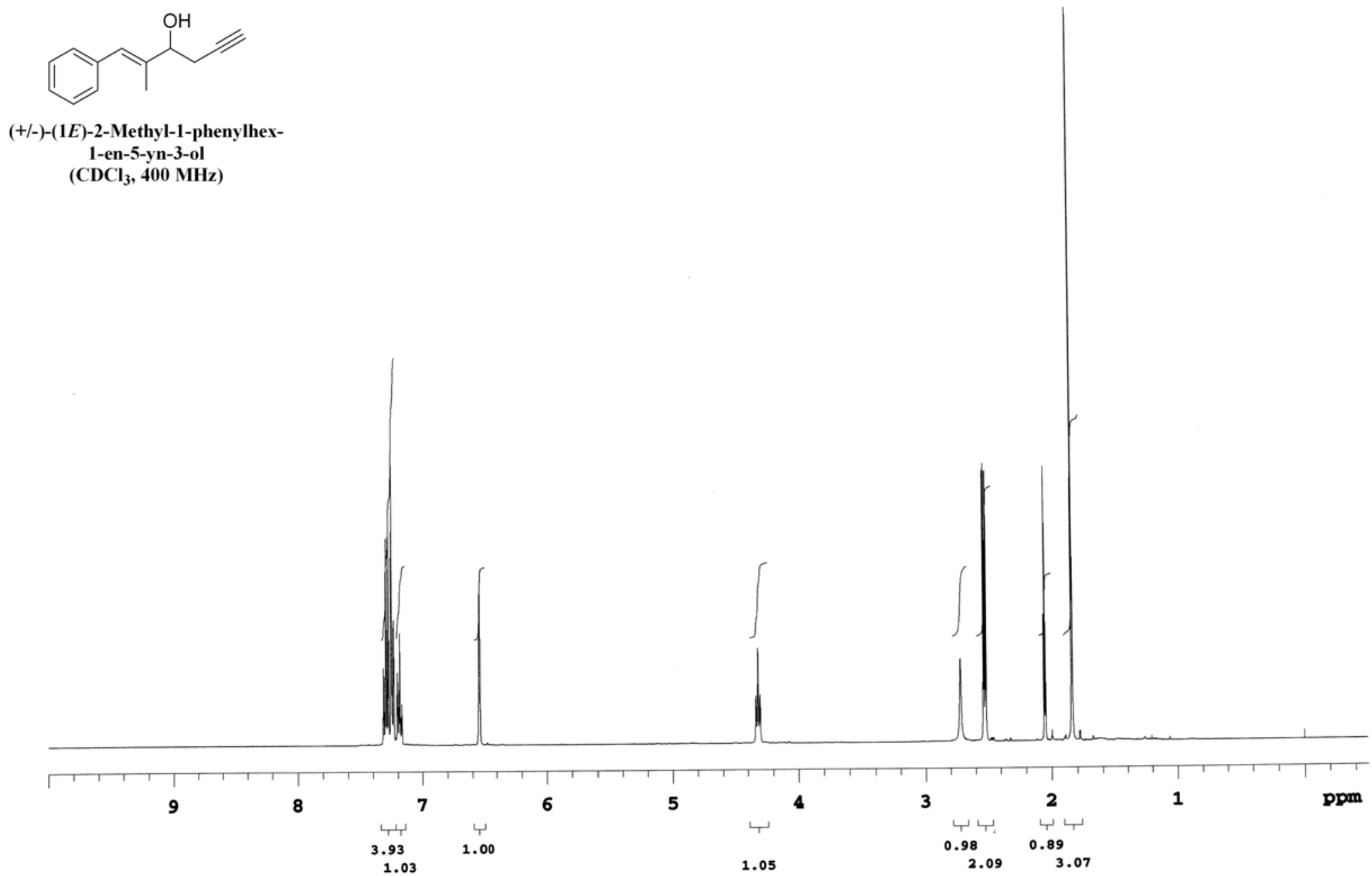


(2): (+/-)-(1E)-2-Methyl-1-phenylhex-1-en-5-yn-3-ol
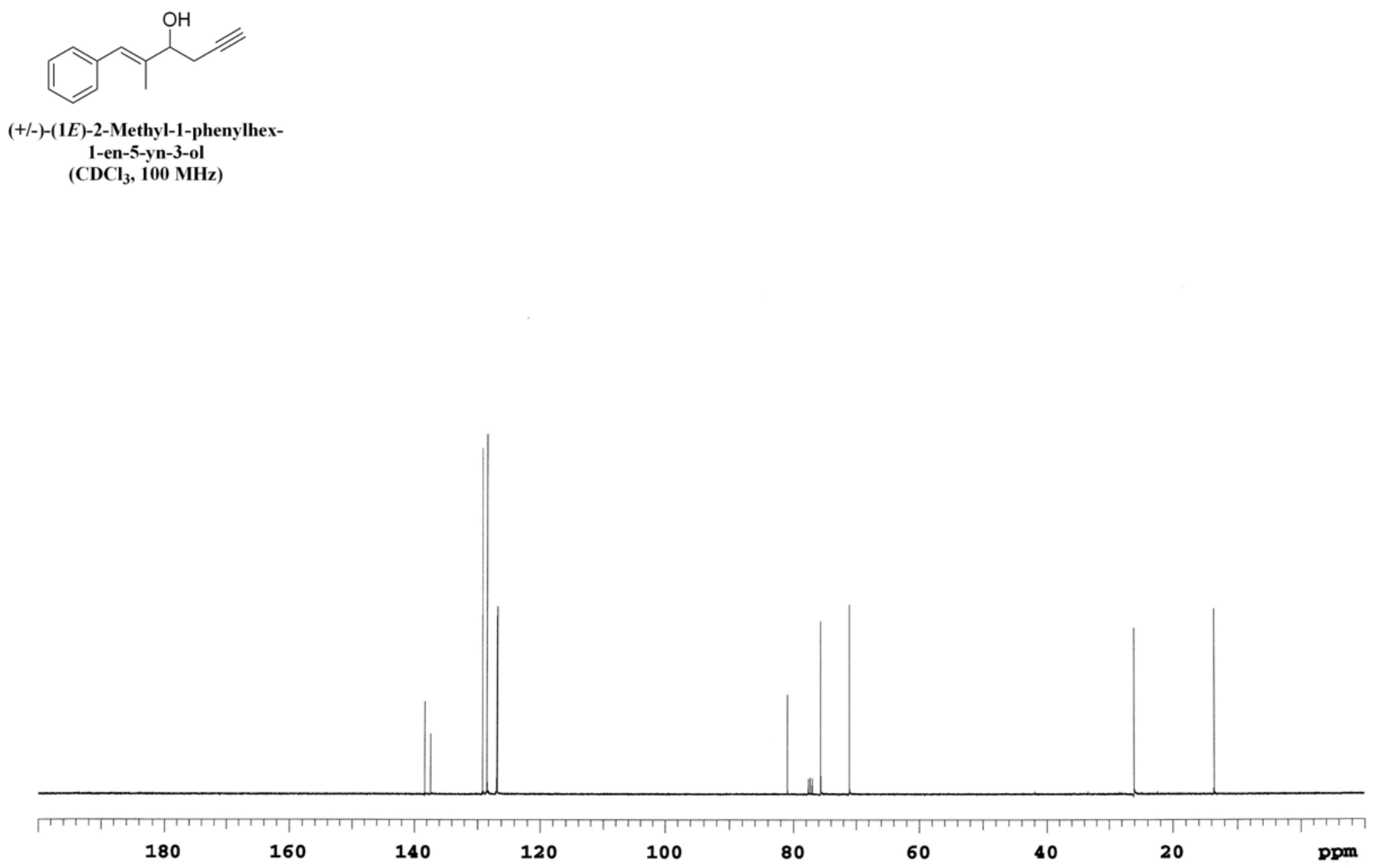
(3): (+/-)-1-Phenylbut-3-yn-1-ol
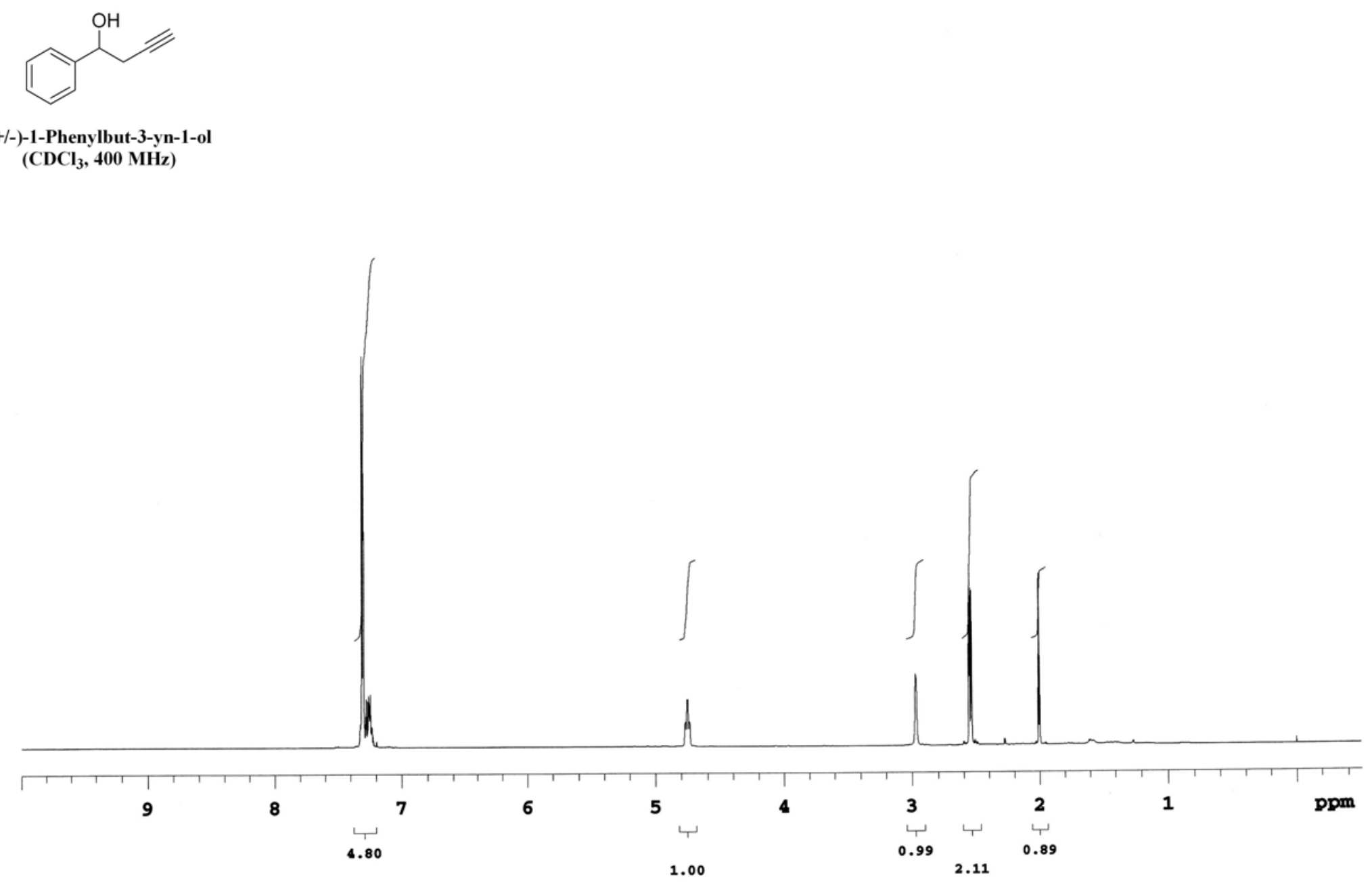
(3): (+/-)-1-Phenylbut-3-yn-1-ol
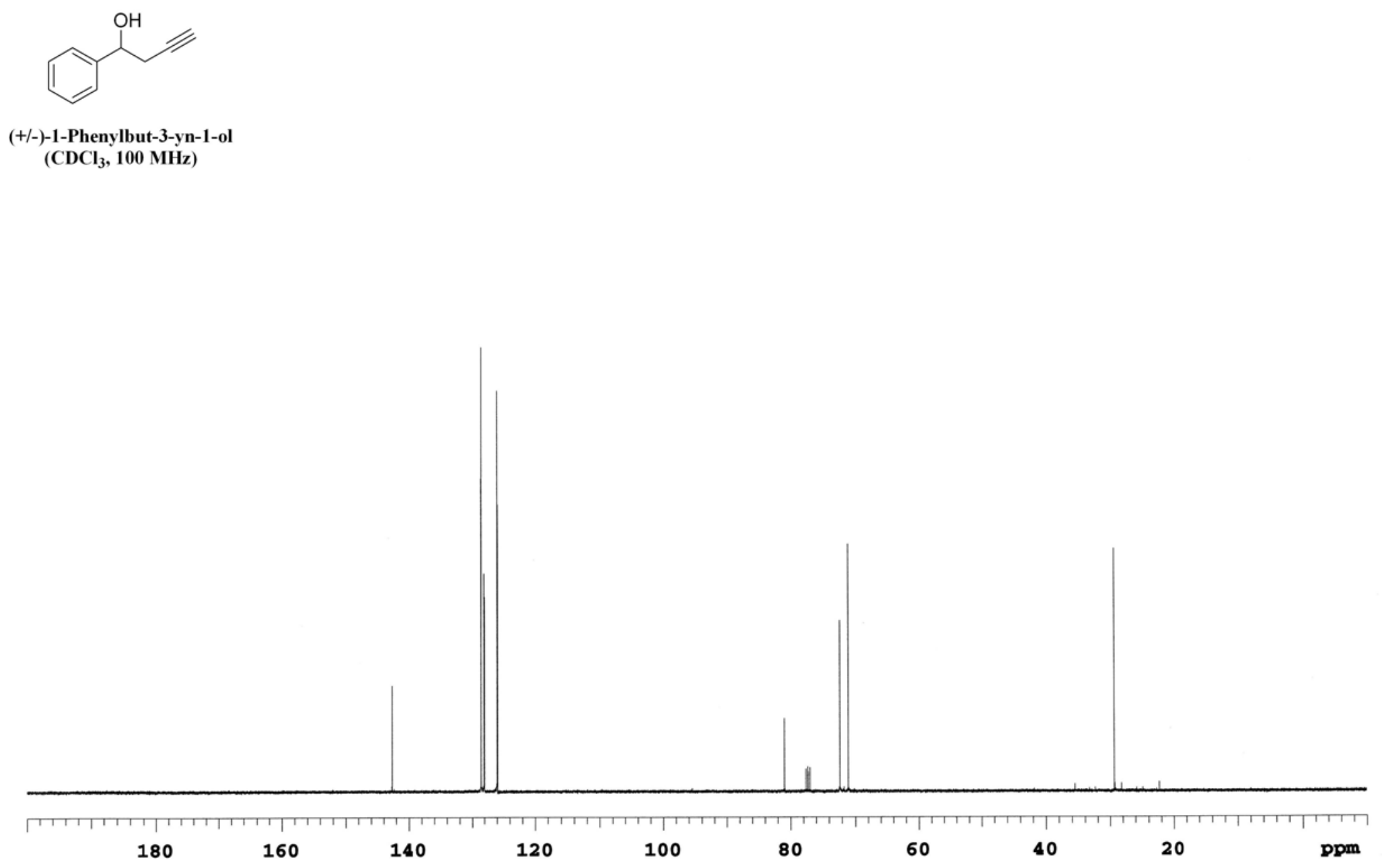
(4): (+/-)-1-Cyclohexylbut-3-yn-1-ol

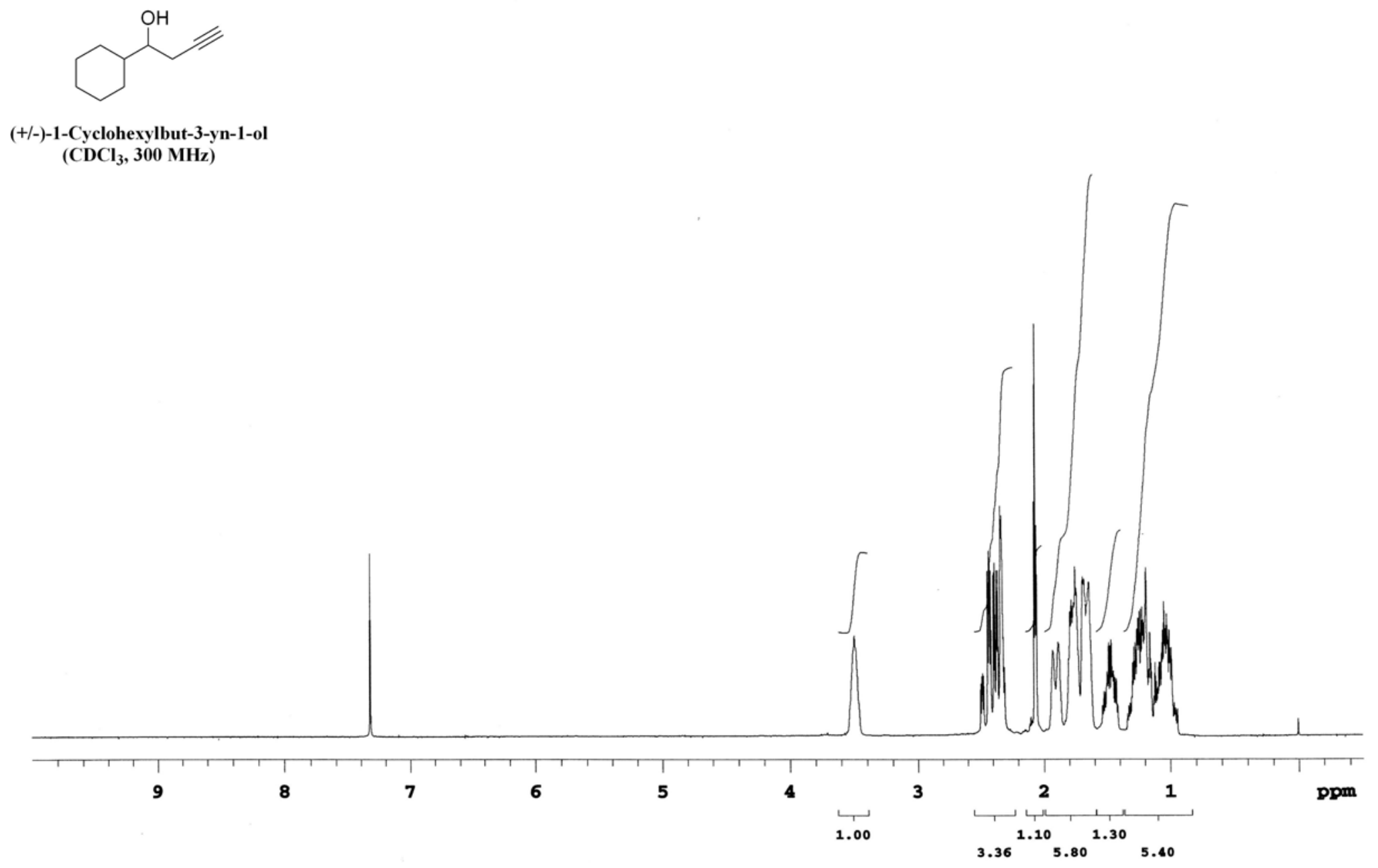


(4): (+/-)-1-Cyclohexylbut-3-yn-1-ol

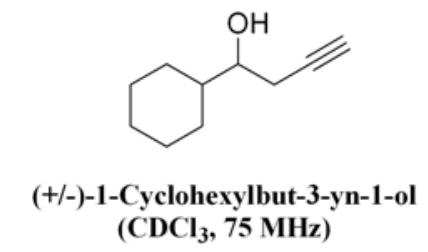

$\left(\mathrm{CDCl}_{3}, 75 \mathrm{MHz}\right)$

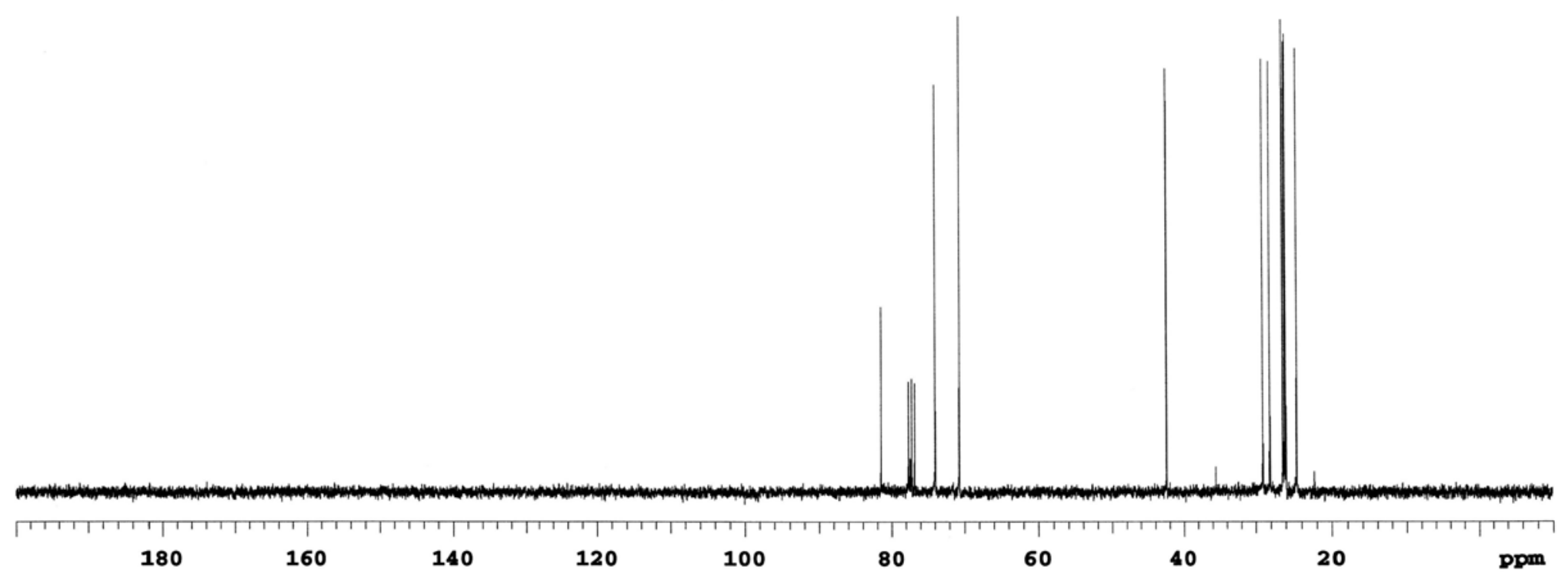


(5): (+/-)-1-(4-Methoxyphenyl)but-3-yn-1-ol

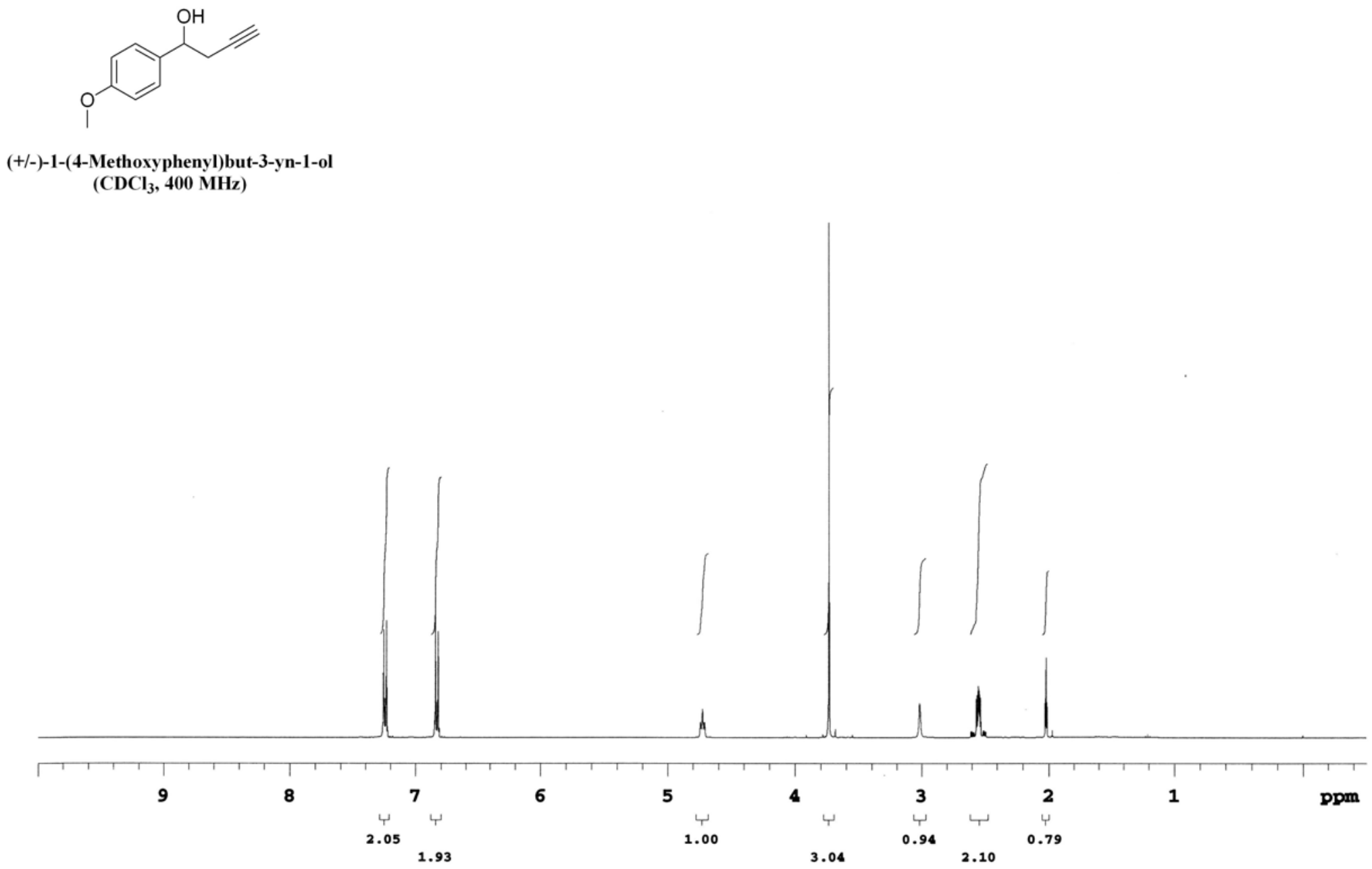


(5): (+/-)-1-(4-Methoxyphenyl)but-3-yn-1-ol
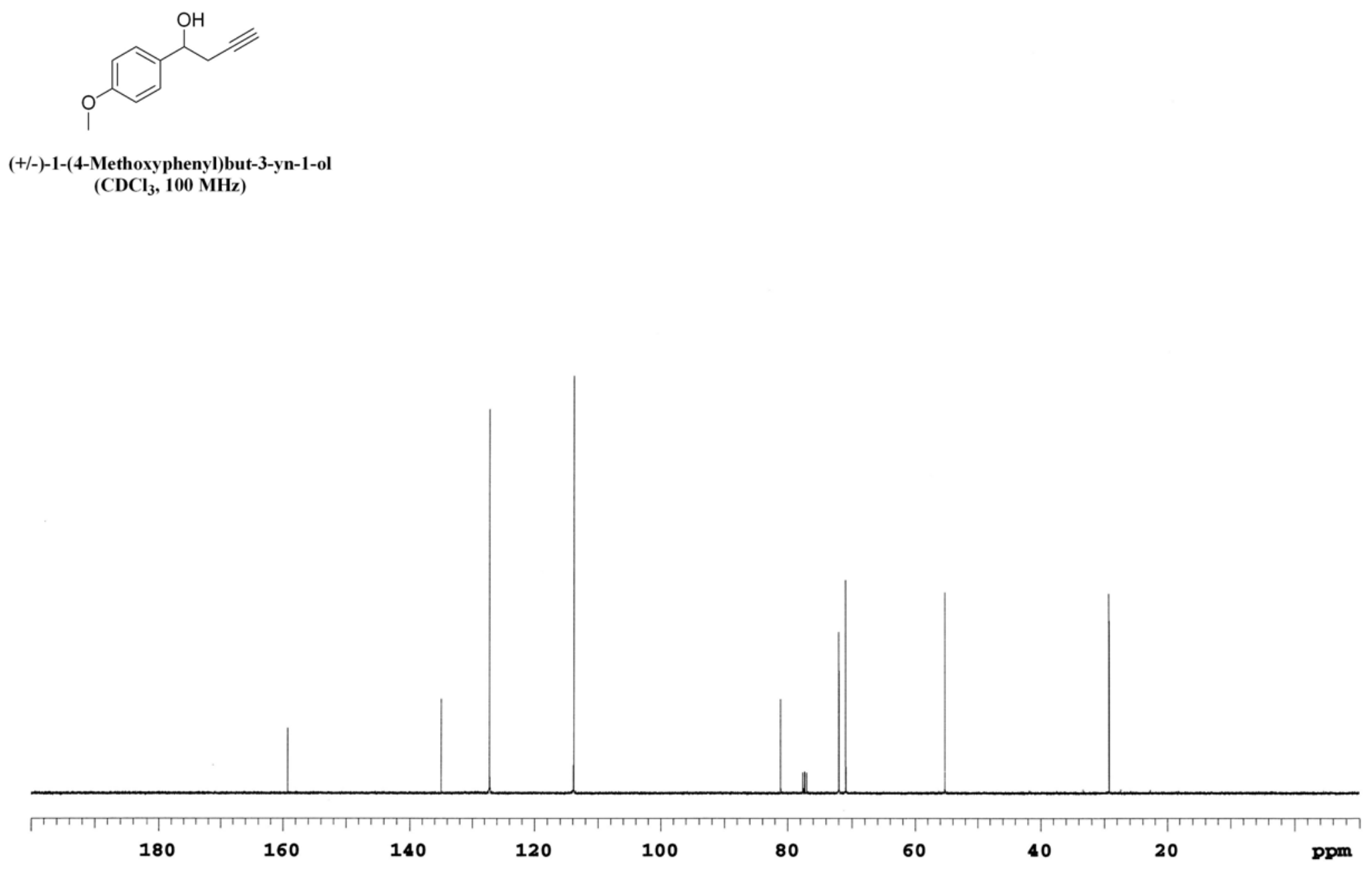
(6): (+/-)-1-(4-Nitrophenyl)but-3-yn-1-ol

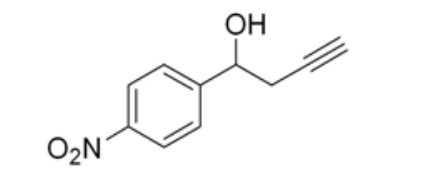

(+/-)-1-(4-Nitrophenyl)but-3-yn-1-ol (d-Acetone, $400 \mathrm{MHz}$ )

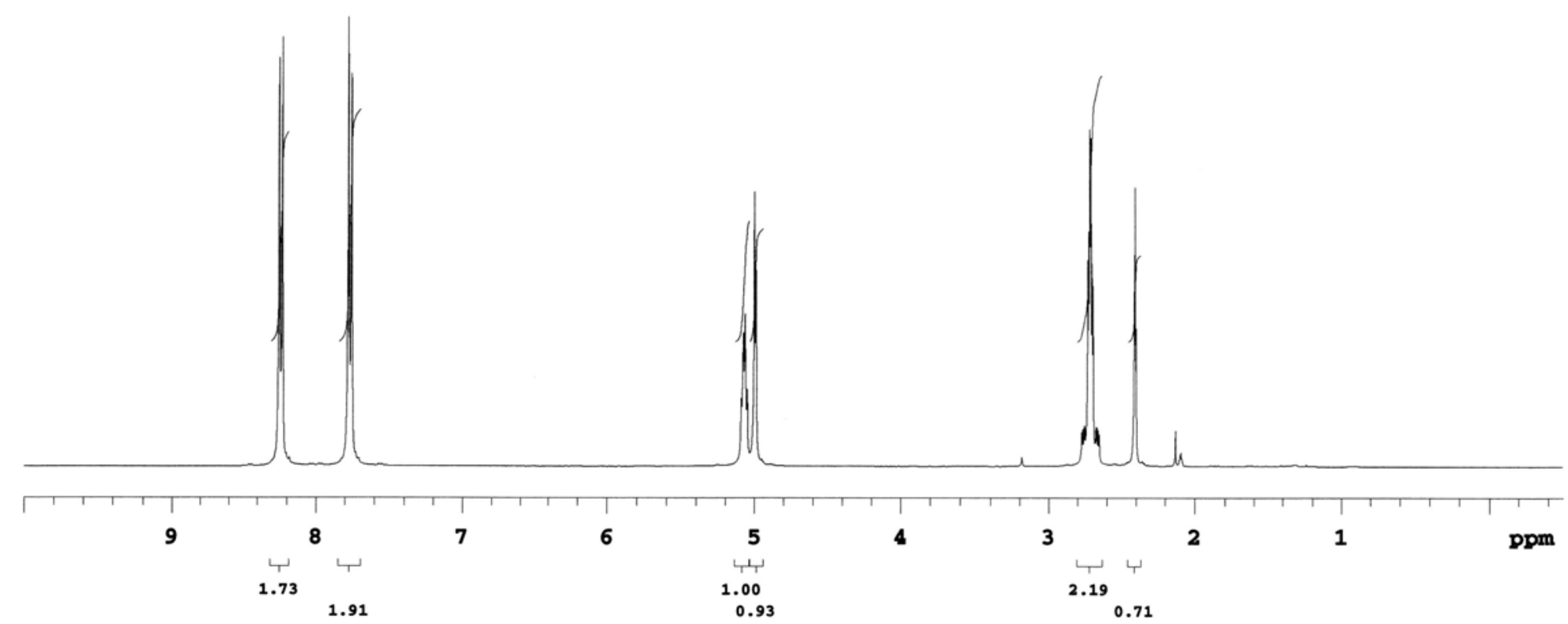


(6): (+/-)-1-(4-Nitrophenyl)but-3-yn-1-ol

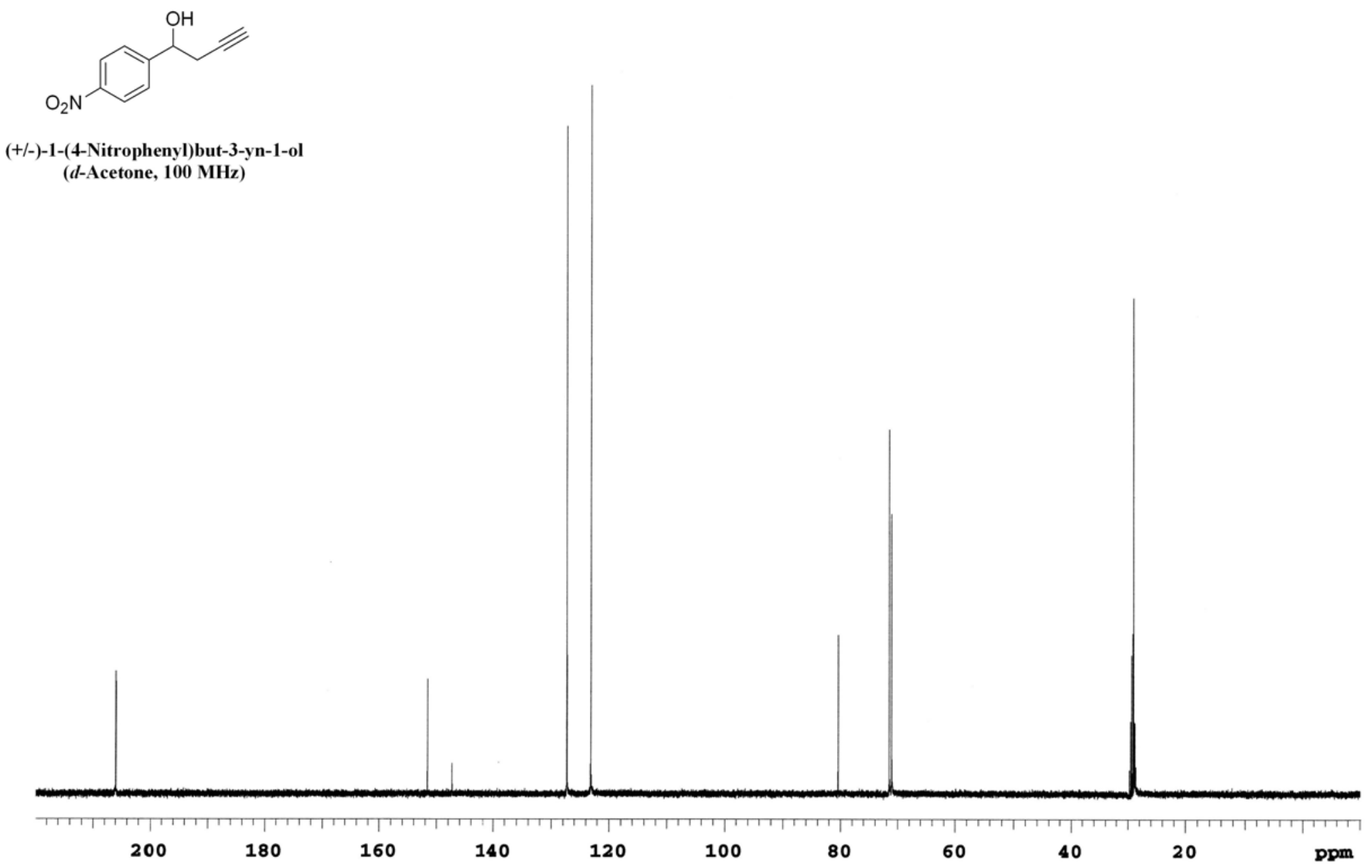


(7): (+/-)-2-Phenylpent-4-yn-2-ol

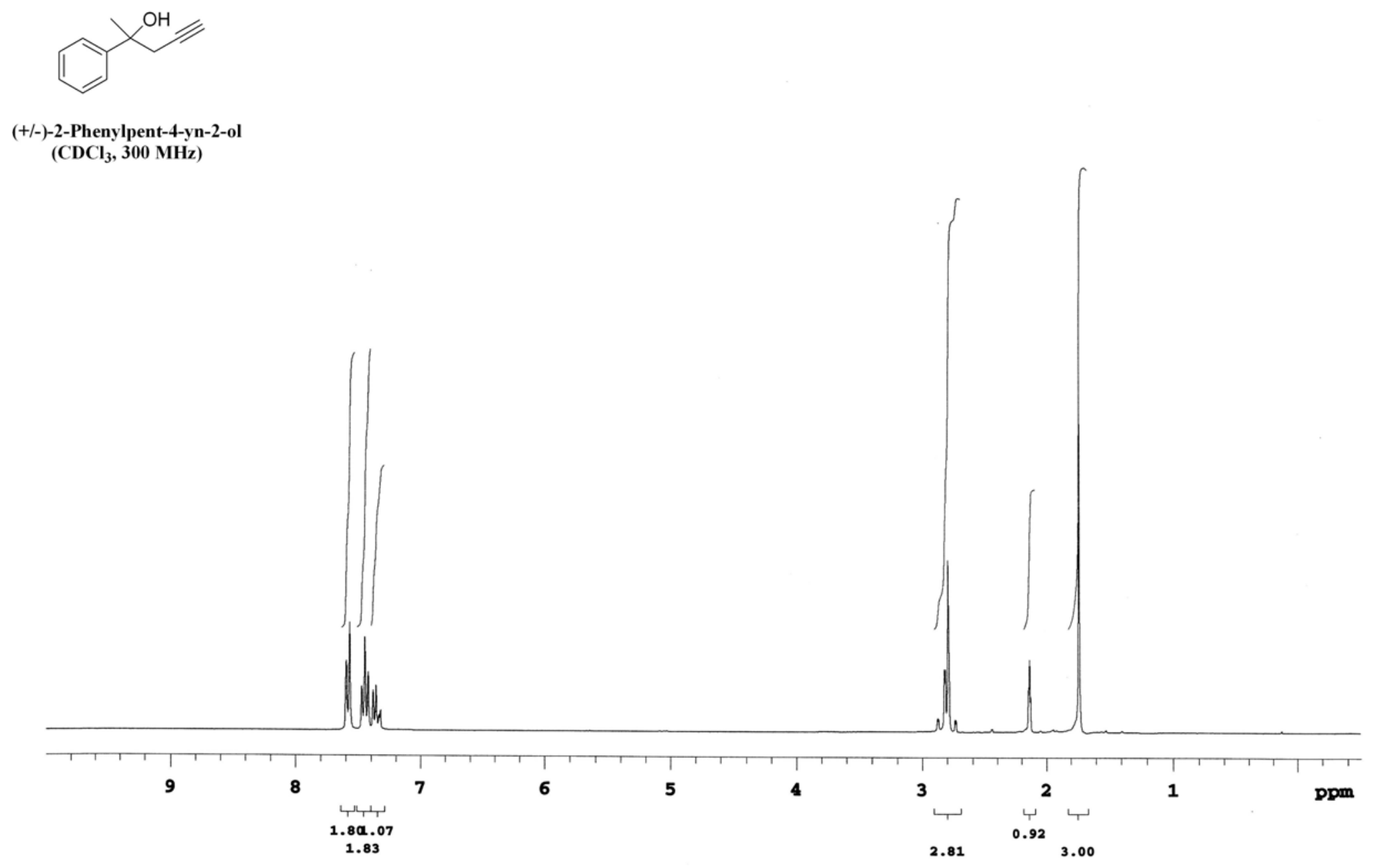


(7): (+/-)-2-Phenylpent-4-yn-2-ol

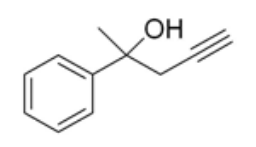

(+/-)-2-Phenylpent-4-yn-2-ol $\left(\mathrm{CDCl}_{3}, 75 \mathrm{MHz}\right)$

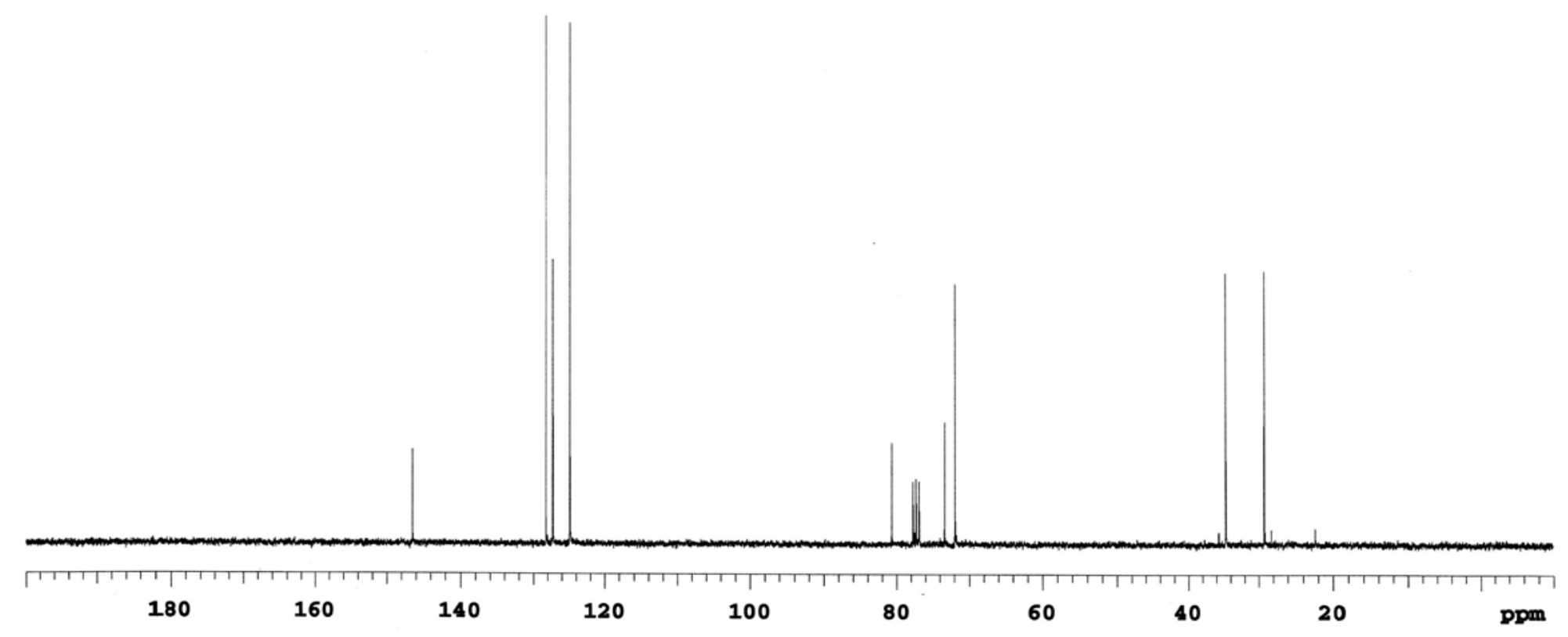


(8): 1-Prop-2-ynylcyclopentanol

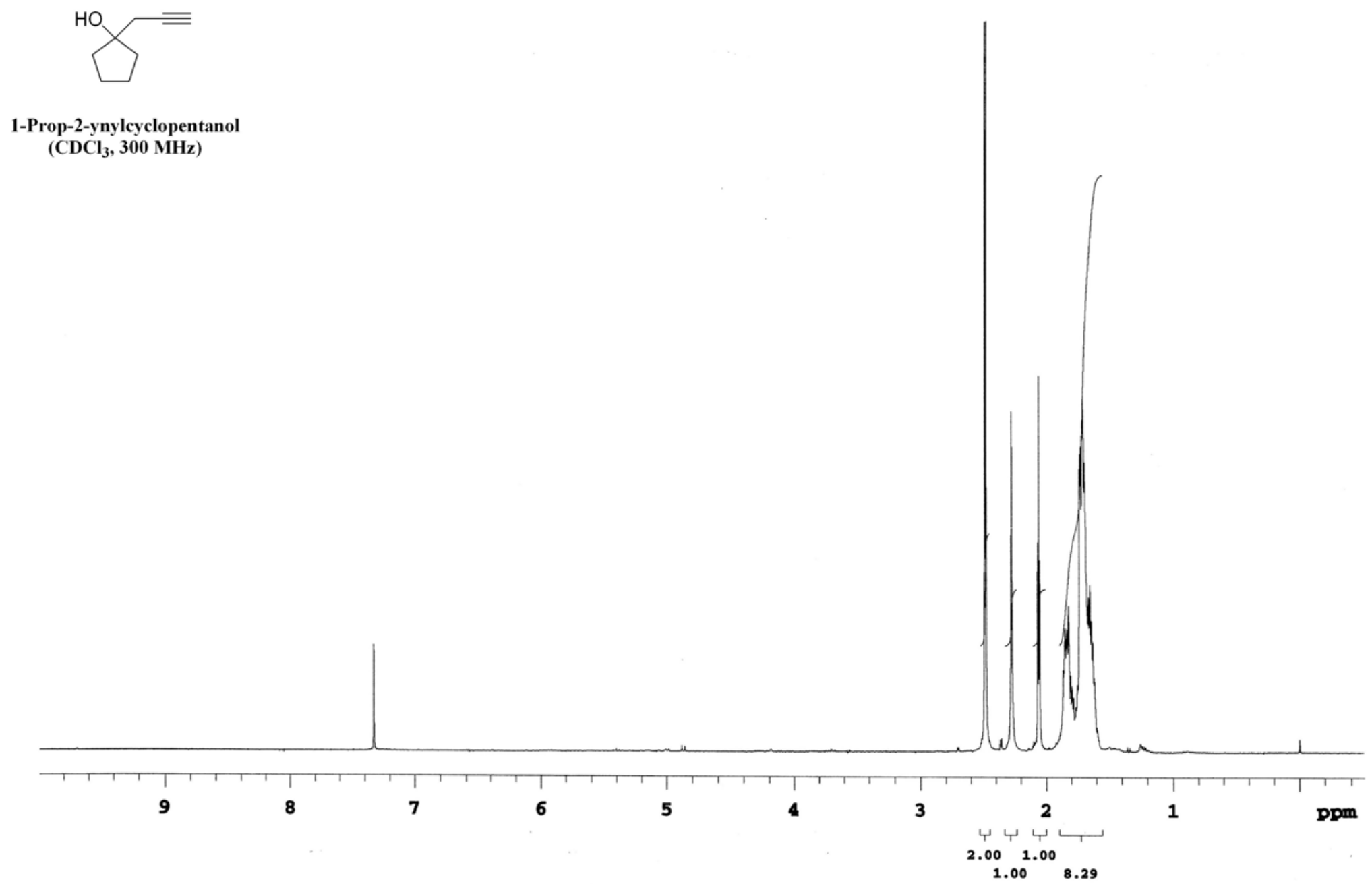


(8): 1-Prop-2-ynylcyclopentanol

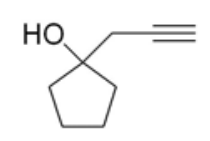

1-Prop-2-ynylcyclopentanol $\left(\mathrm{CDCl}_{3}, 75 \mathrm{MHz}\right)$

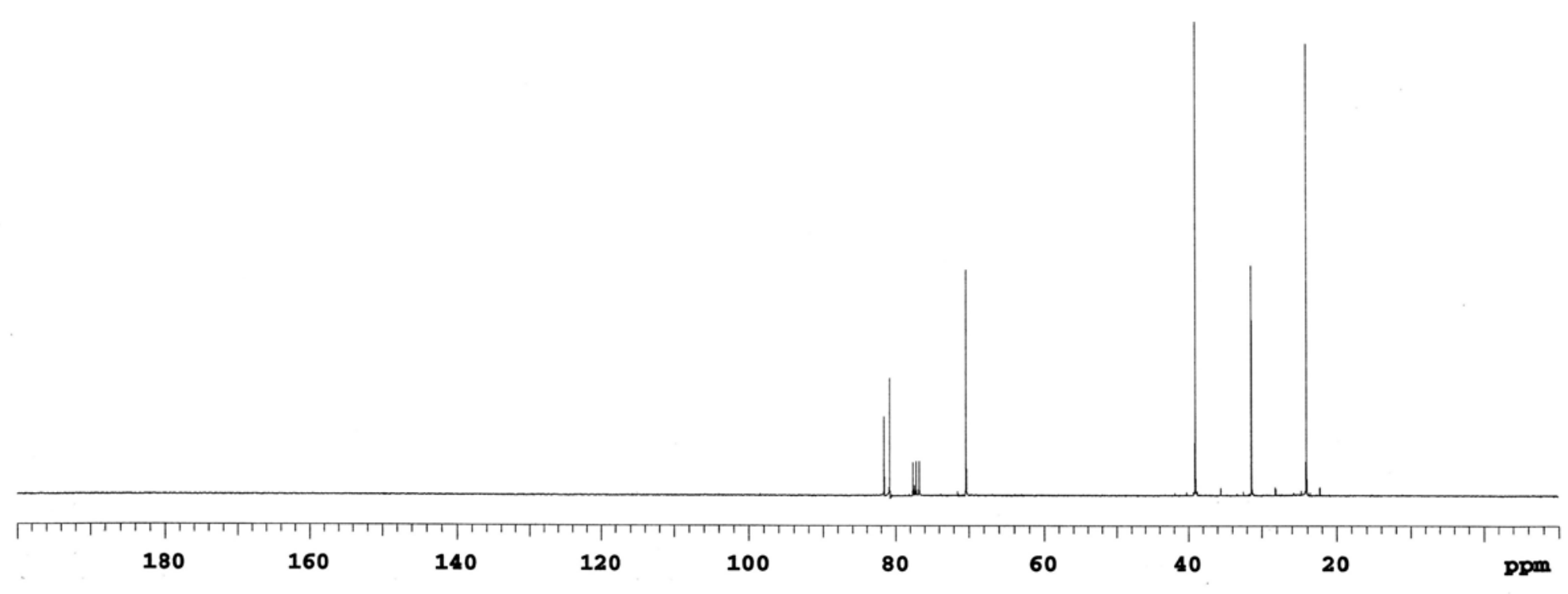




\section{(9): 1-Prop-2-ynylcyclohexanol}

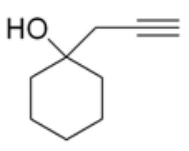

(+/-)-1-Prop-2-ynylcyclohexanol $\left(\mathrm{CDCl}_{3}, 300 \mathrm{MHz}\right)$

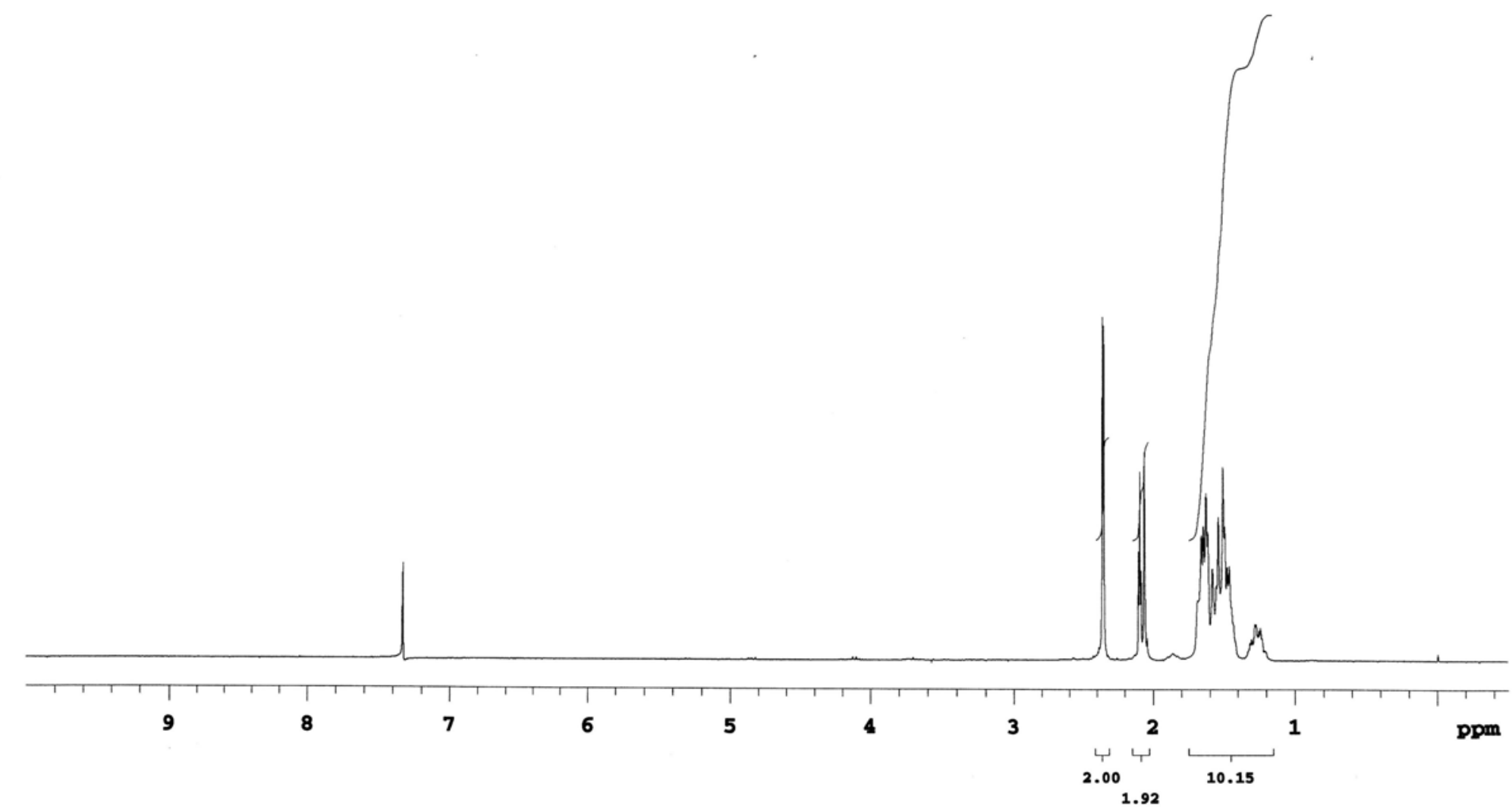


(9): 1-Prop-2-ynylcyclohexanol

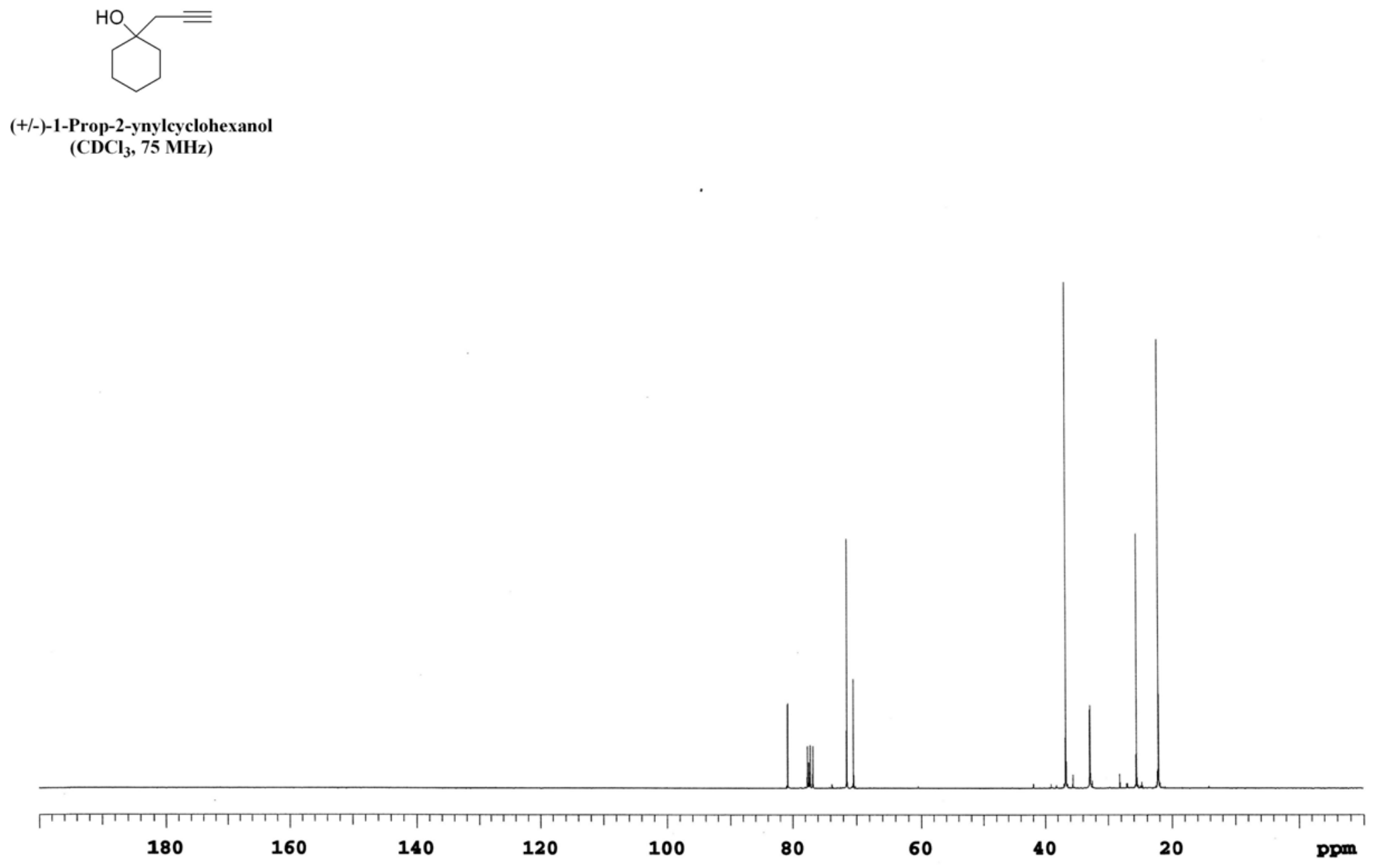


(10): (+/-)-2,2,3-Trimethylhex-5-yn-3-ol

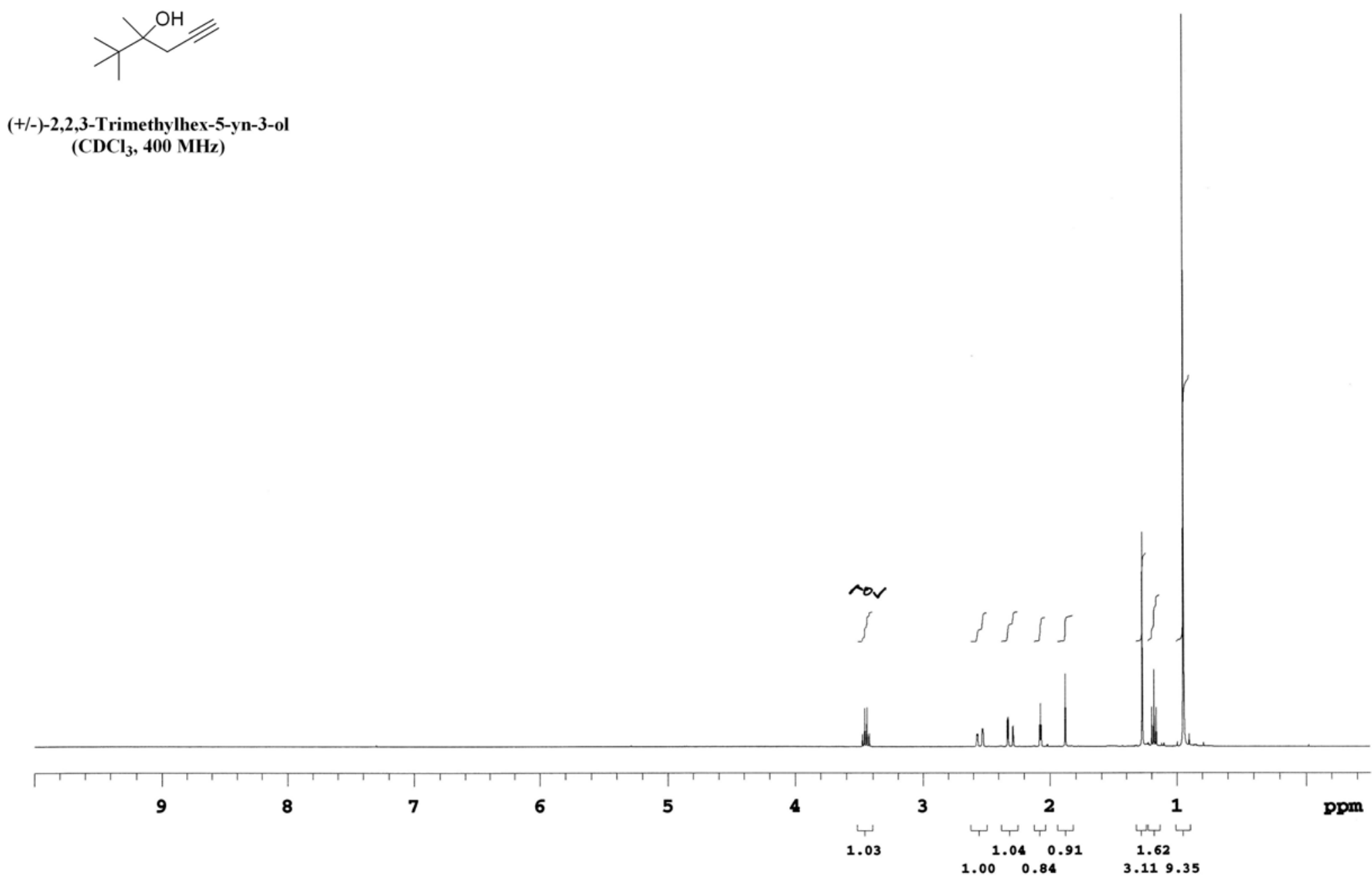


(10): (+/-)-2,2,3-Trimethylhex-5-yn-3-ol

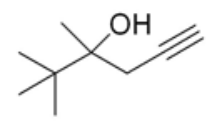

(+/-)-2,2,3-Trimethylhex-5-yn-3-ol $\left(\mathrm{CDCl}_{3}, 100 \mathrm{MHz}\right)$

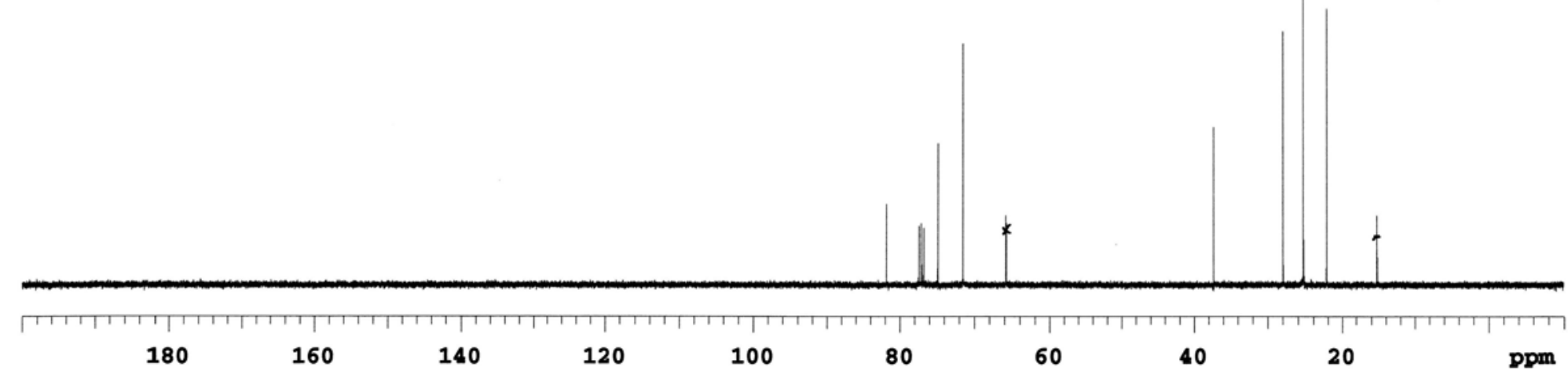


(12): (+/-)-(1E)-2-Methyl-1-phenylhept-1-en-6-yn-4-ol

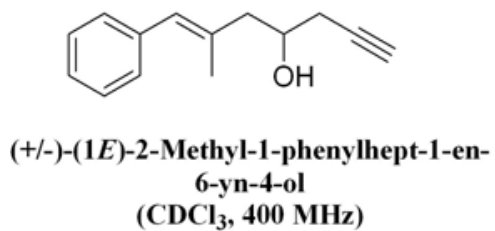

$\left(\mathrm{CDCl}_{3}, 400 \mathrm{MHz}\right)$

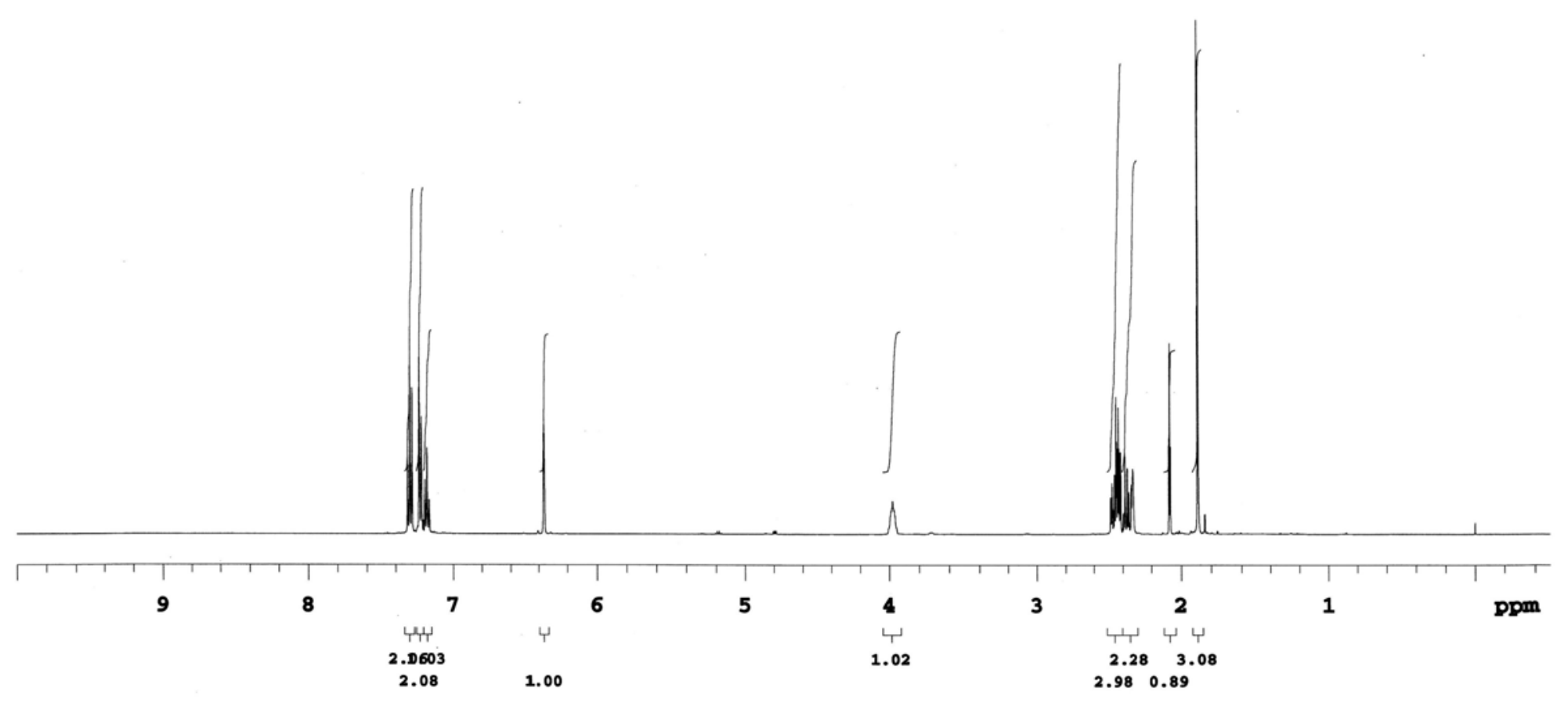


(12): (+/-)-(1E)-2-Methyl-1-phenylhept-1-en-6-yn-4-ol

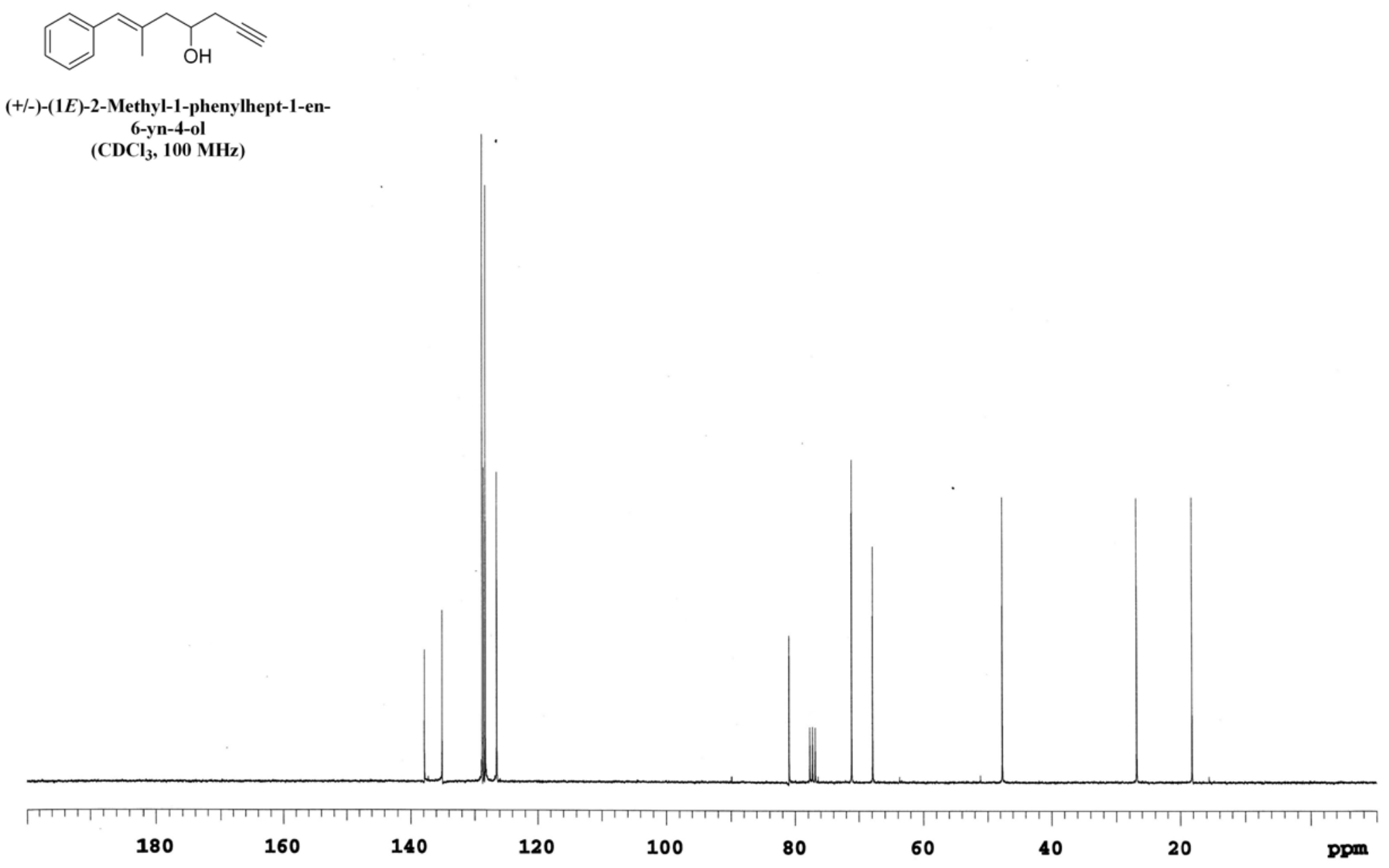


(14): (+/-)-1,1-Diphenylhept-1-en-6-yn-4-ol

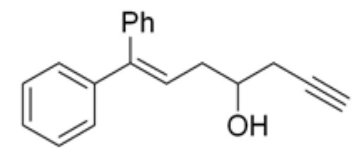

$$
\begin{aligned}
& \text { (+/-)-1,1-Diphenylhept-1-en-6-yn-4-ol } \\
& \text { (CDCl } 3,400 \mathrm{MHz})
\end{aligned}
$$

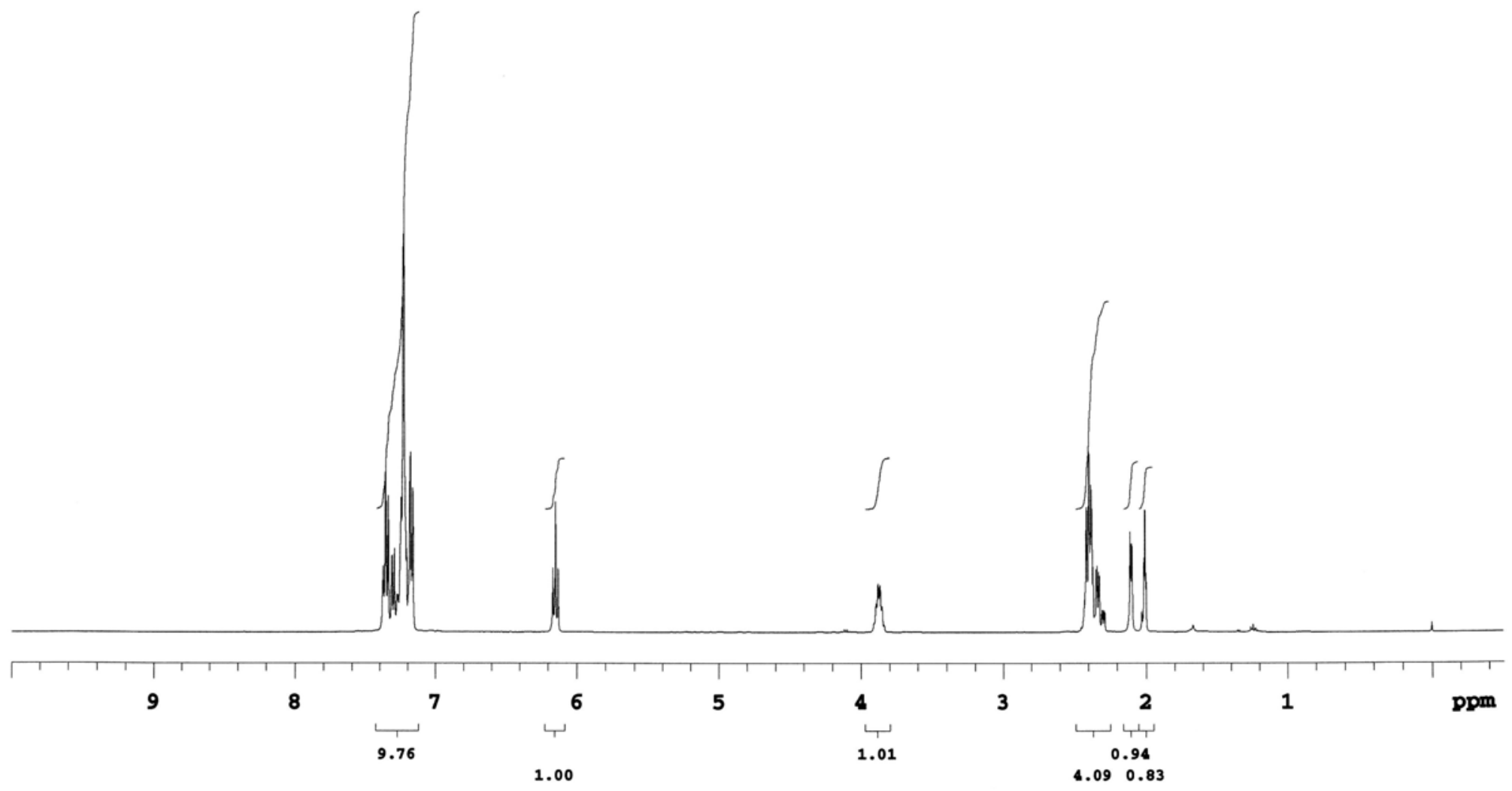

-SF 22 - 
(14): (+/-)-1,1-Diphenylhept-1-en-6-yn-4-ol

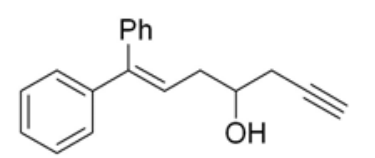

(+/-)-1,1-Diphenylhept-1-en-6-yn-4-ol $\left(\mathrm{CDCl}_{3}, 100 \mathrm{MHz}\right)$

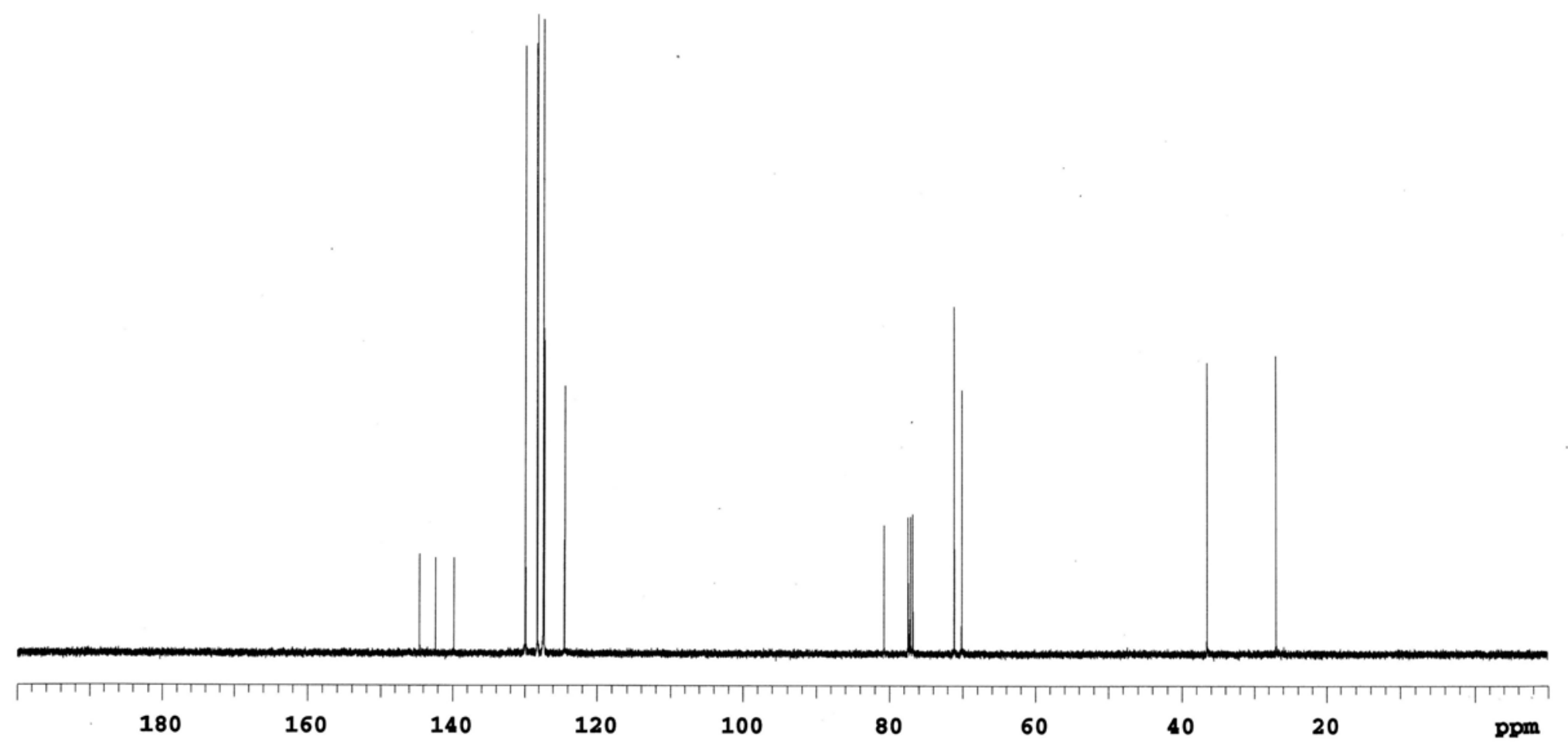




\section{(S-14): (4S)-1,1-Diphenylhept-1-en-6-yn-4-ol}

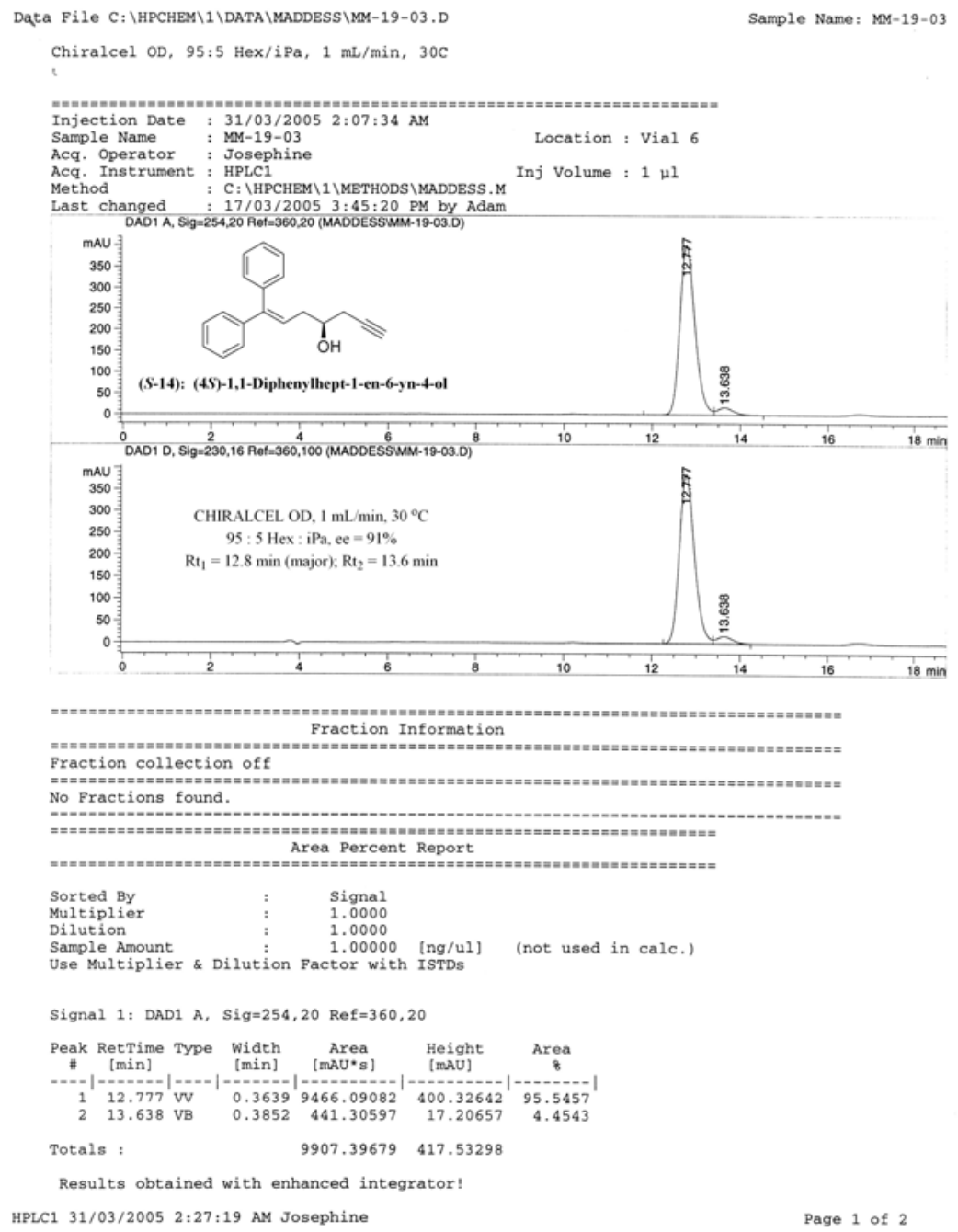


(R-14): (4S)-1,1-Diphenylhept-1-en-6-yn-4-ol

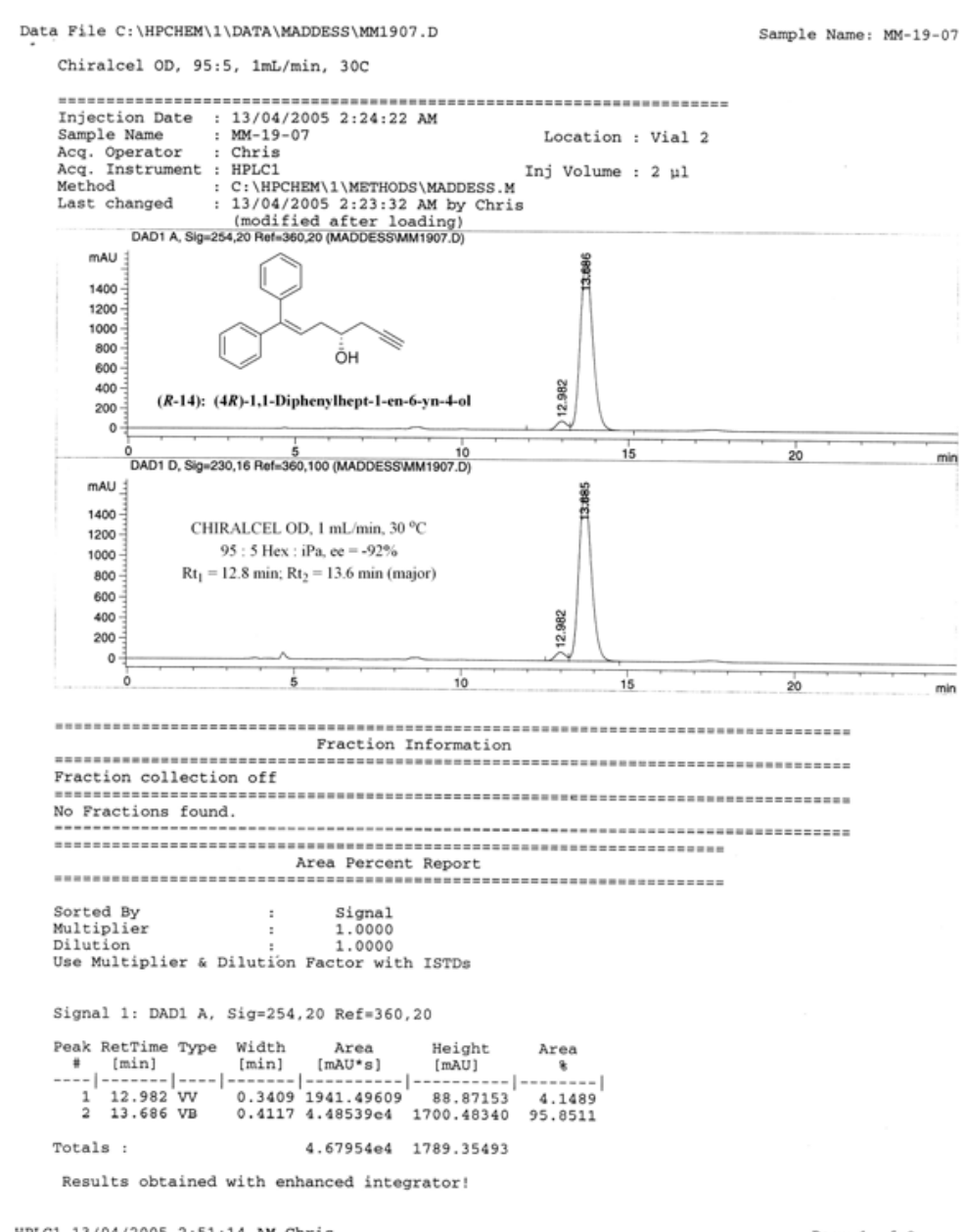

HPLC1 13/04/2005 2:51:14 AM Chris 
(Sup 1): (1R)-4,4-Diphenyl-1-prop-2-ynylbut-3-enyl 4-nitrobenzoate

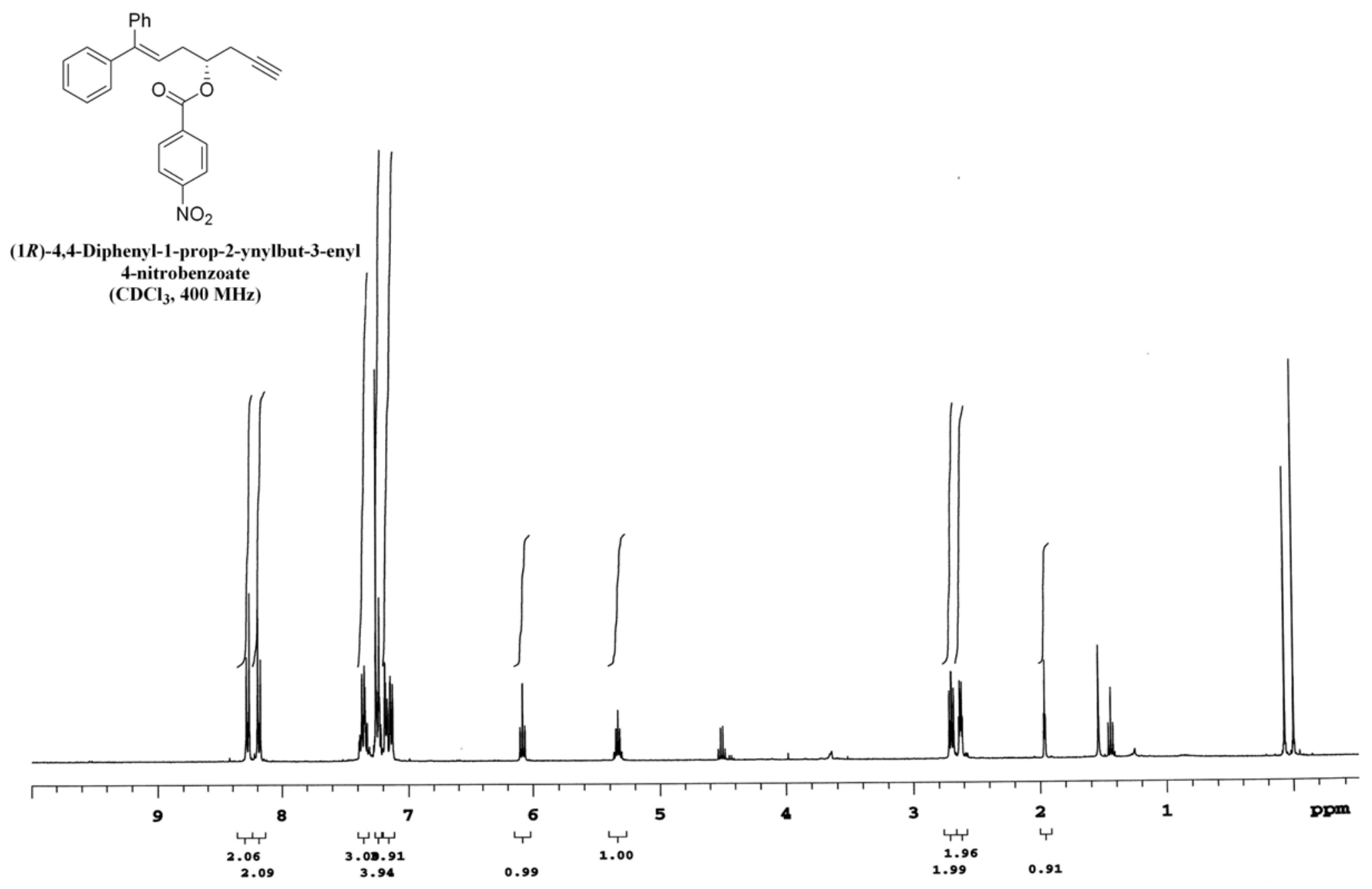


(Sup 1): (1R)-4,4-Diphenyl-1-prop-2-ynylbut-3-enyl 4-nitrobenzoate

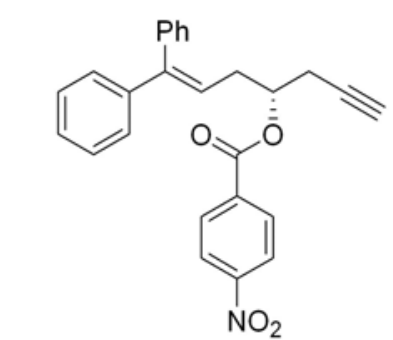

$R$ )-4,4-Diphenyl-1-prop-2-ynylbut-3-enyl

4-nitrobenzoate

$\left(\mathrm{CDCl}_{3}, 100 \mathrm{MHz}\right)$

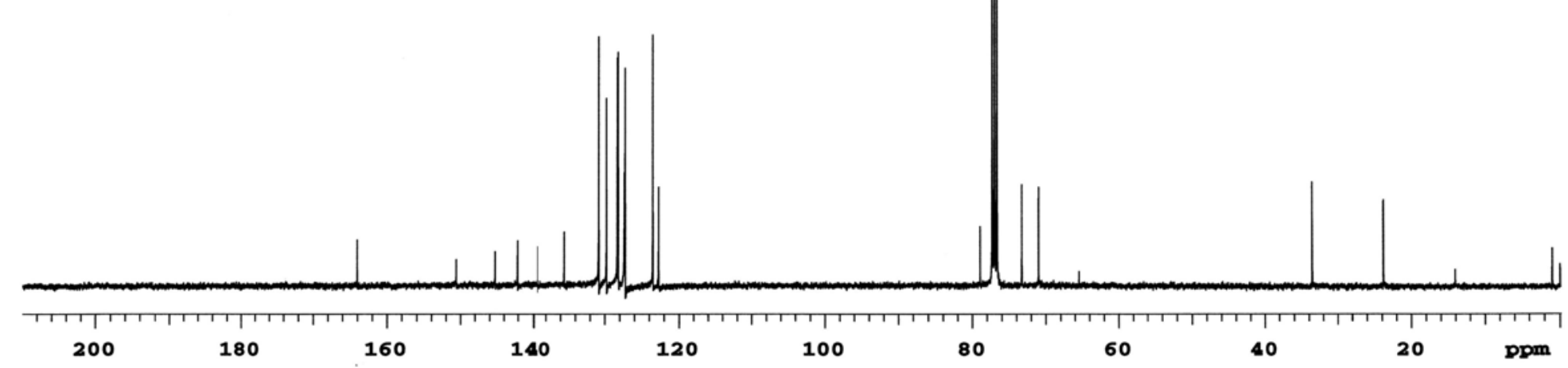




\section{(ent-Sup 2): (4S)-1,1-Diphenylhepta-1,6-dien-4-ol}

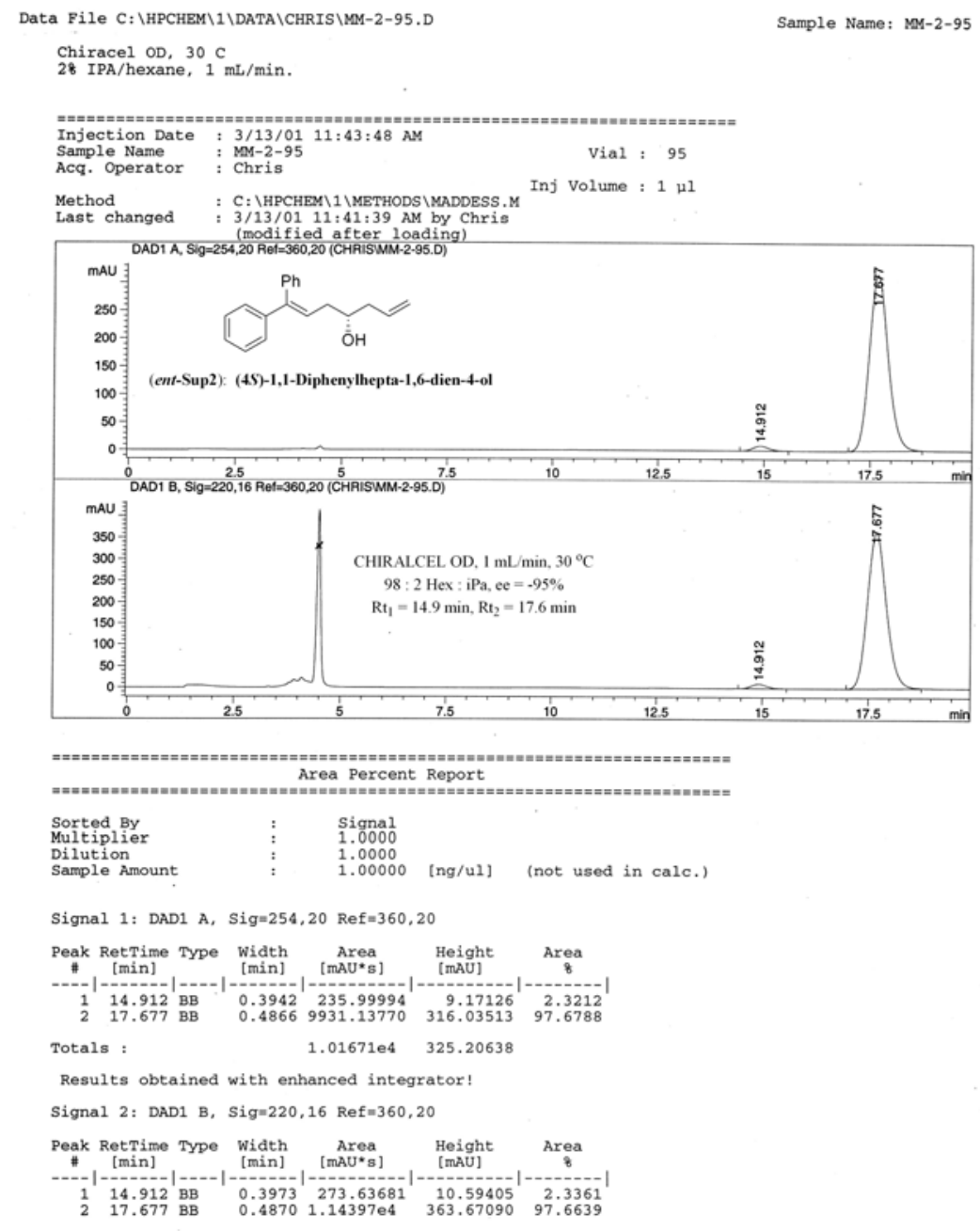

Instrument 13/13/01 12:05:50 PM Chris

Page 1 of 2 
(16): (+/-)-(1Z)-2-Bromo-1-phenylhept-1-en-6-yn-4-ol

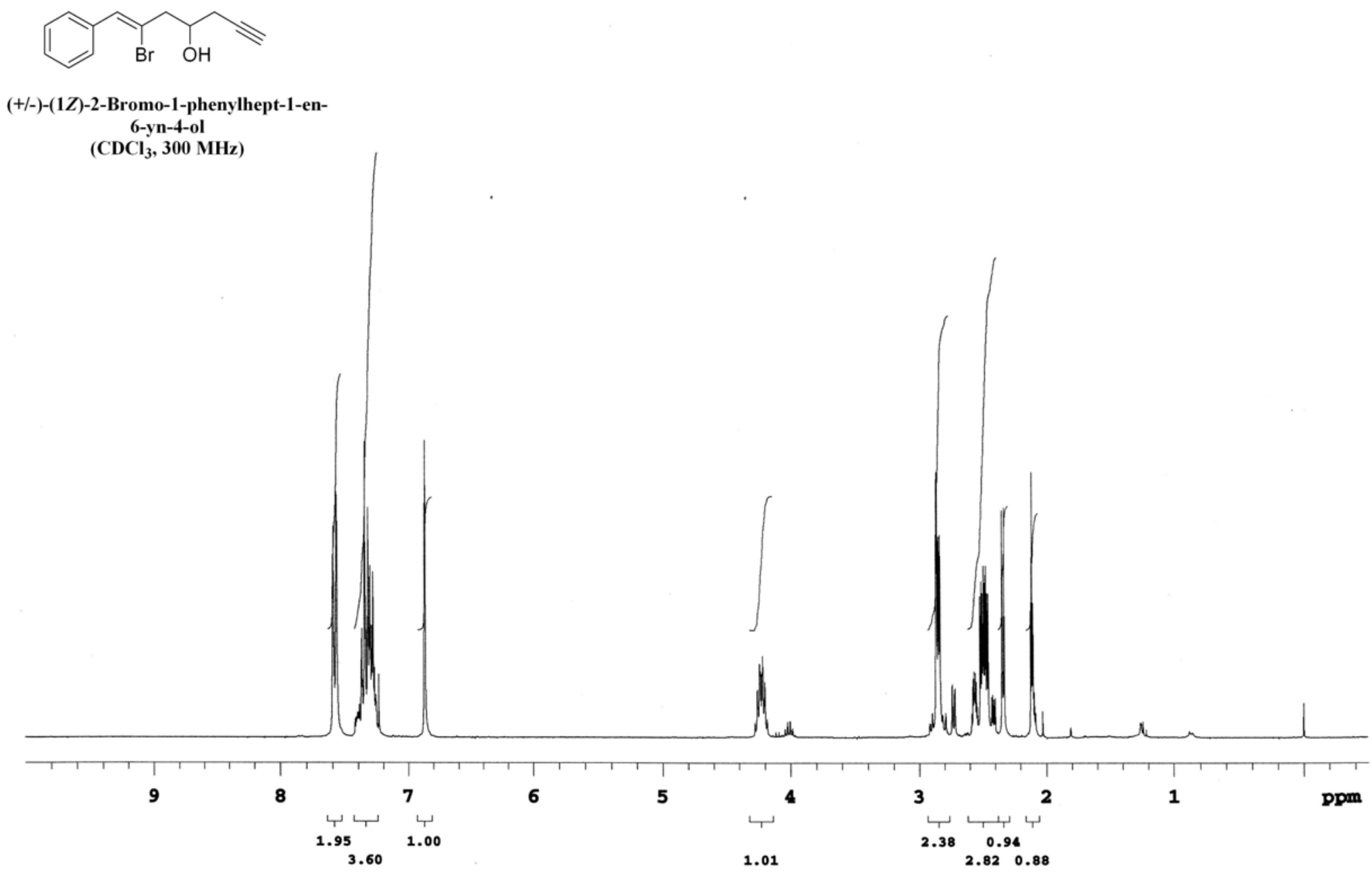


(16): (+/-)-(1Z)-2-Bromo-1-phenylhept-1-en-6-yn-4-ol

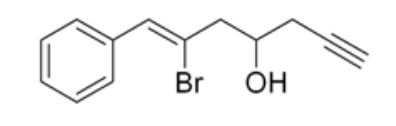

(+/-)-(1Z)-2-Bromo-1-phenylhept-1-en-
6-yn-4-ol

$\left(\mathrm{CDCl}_{3}, 75 \mathrm{MHz}\right)$

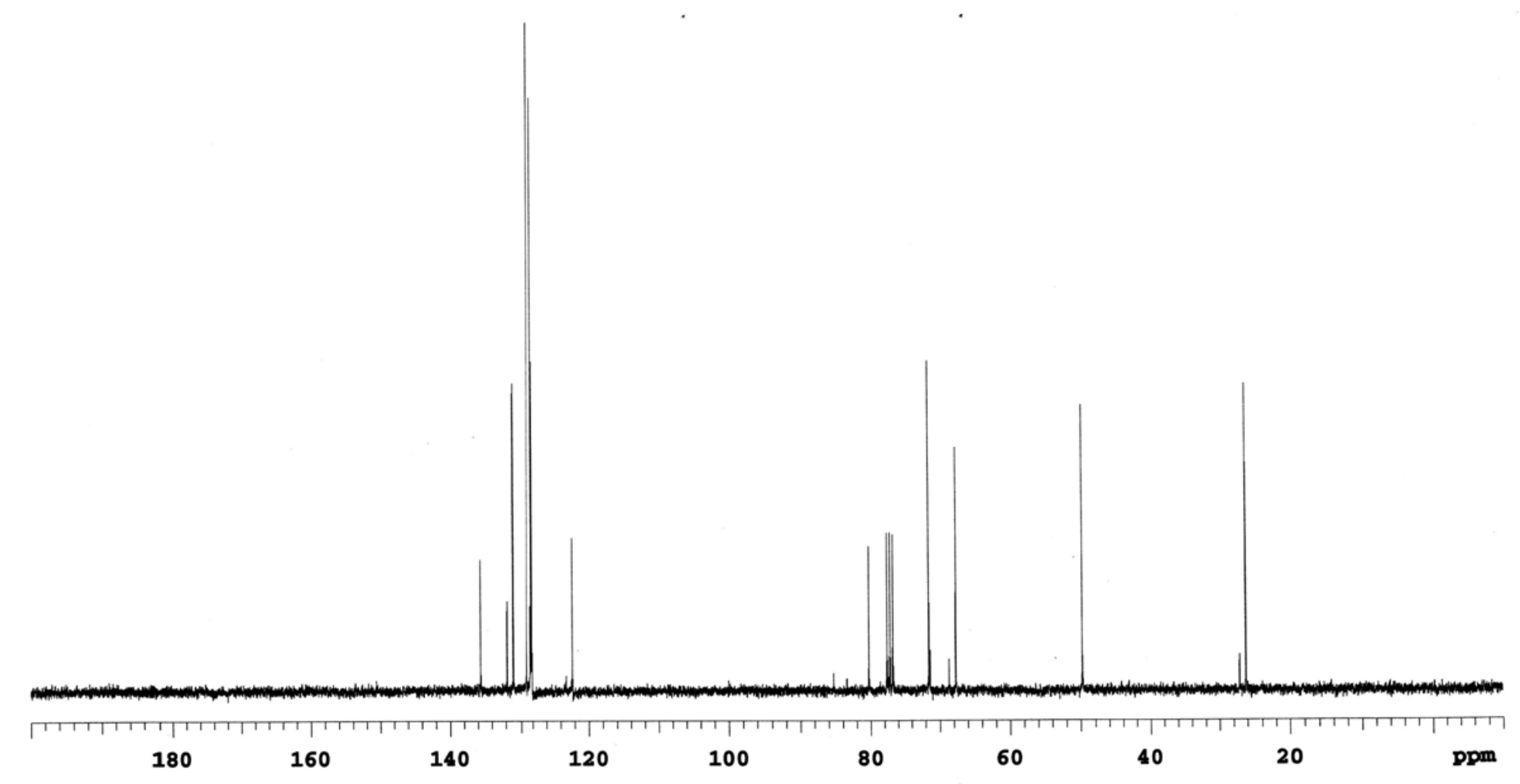


(18): (+/-)-(1E)-1-(2-Methoxyphenyl)hept-1-en-6-yn-4-ol

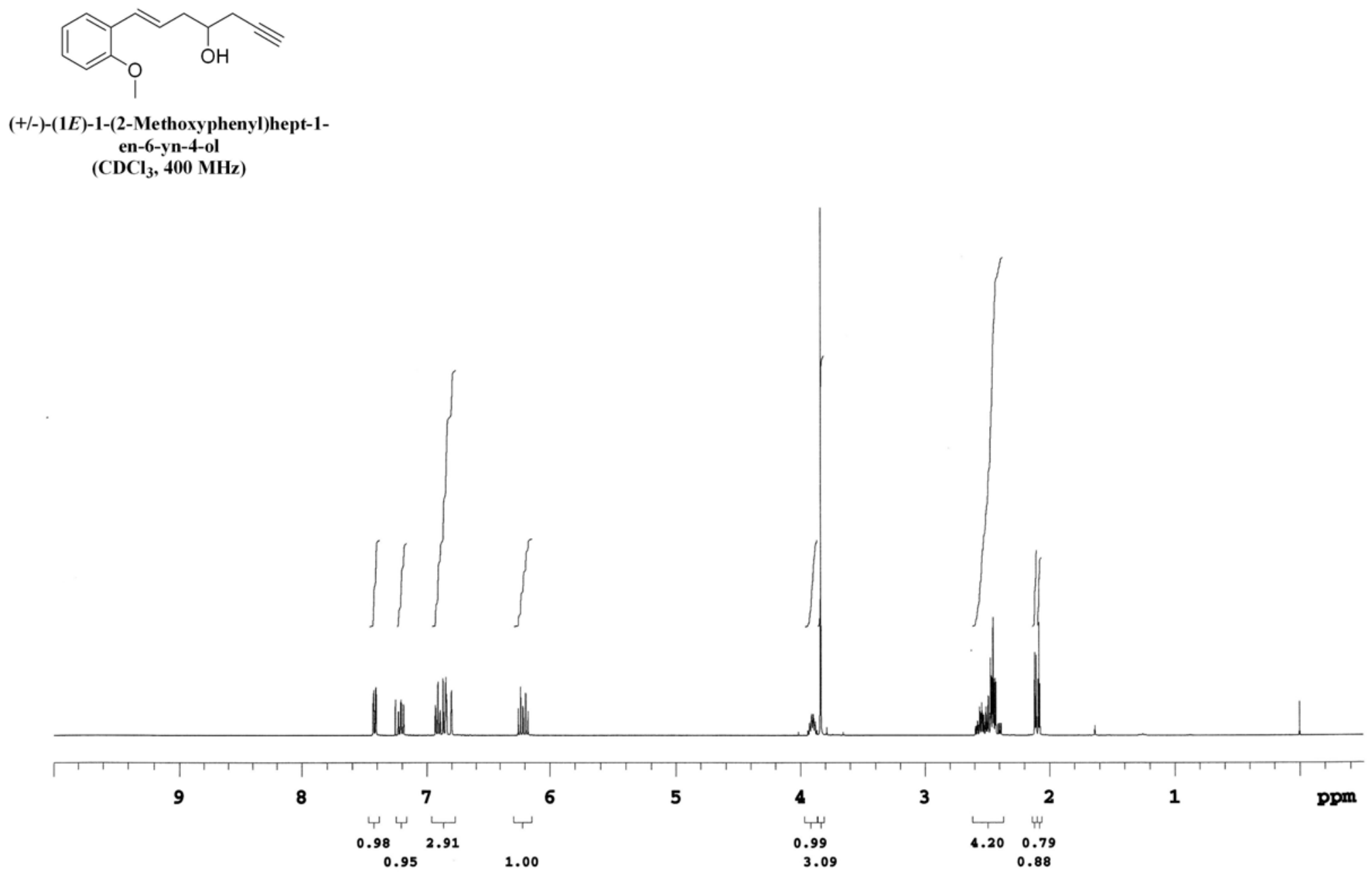


(18): (+/-)-(1E)-1-(2-Methoxyphenyl)hept-1-en-6-yn-4-ol
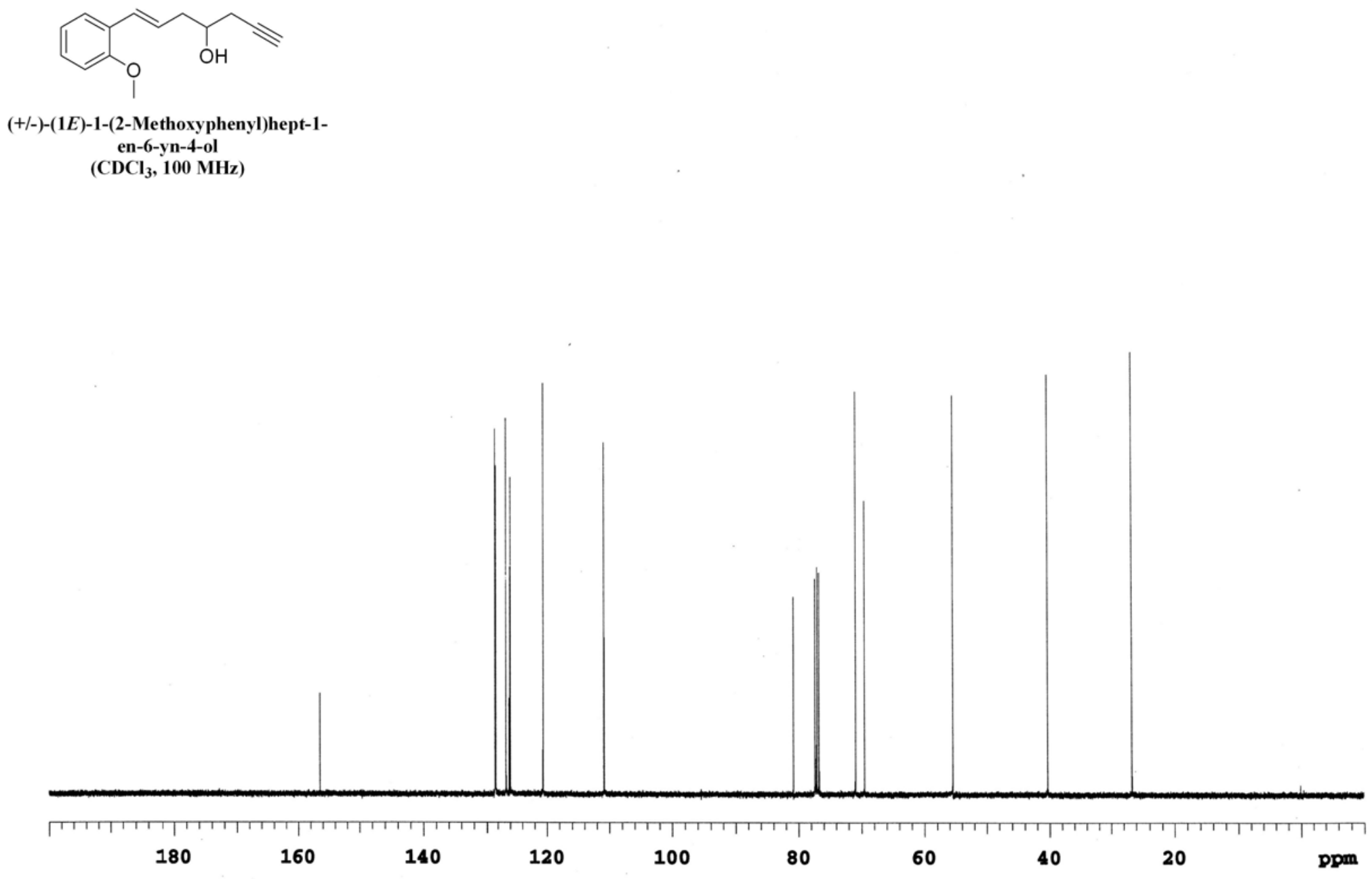
(20): (+/-)-(1E)-1-(2-Nitrophenyl)hept-1-en-6-yn-4-ol

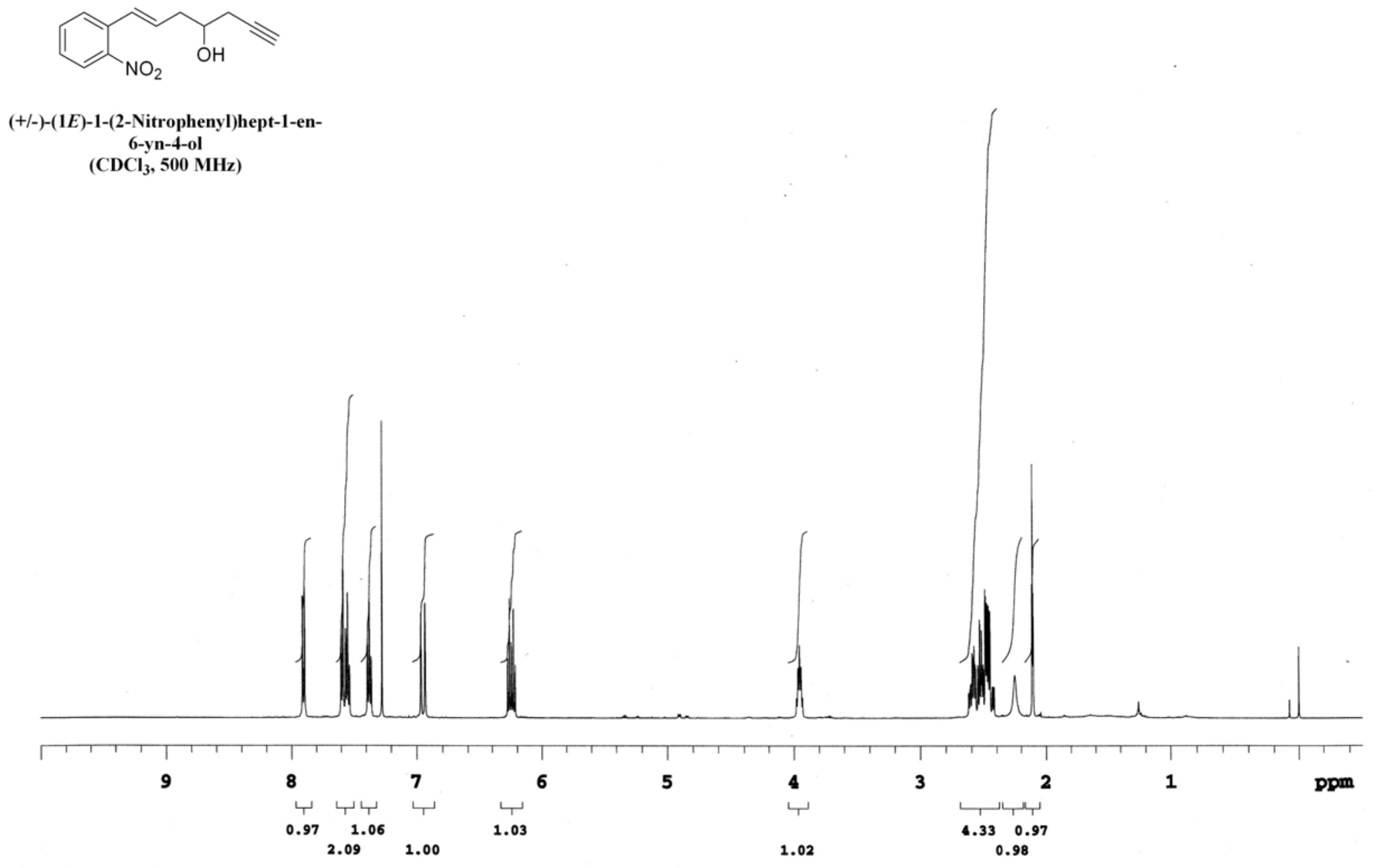


(20): (+/-)-(1E)-1-(2-Nitrophenyl)hept-1-en-6-yn-4-ol

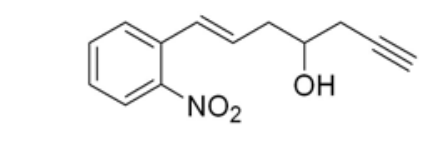

(+/-)-(1E)-1-(2-Nitrophenyl)hept-1-en-

$$
\text { 6-yn-4-ol }
$$

$\left(\mathrm{CDCl}_{3}, 125 \mathrm{MHz}\right)$

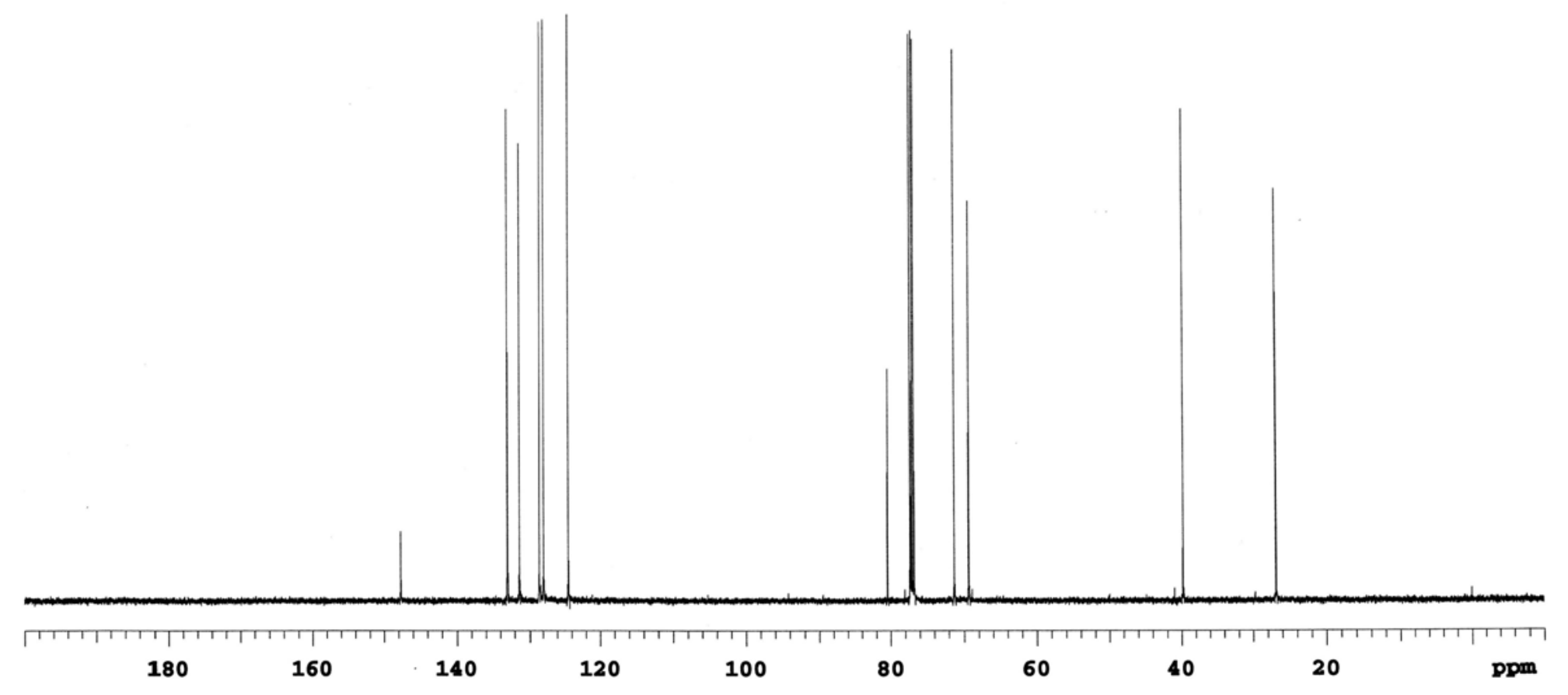




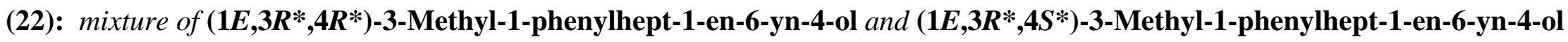

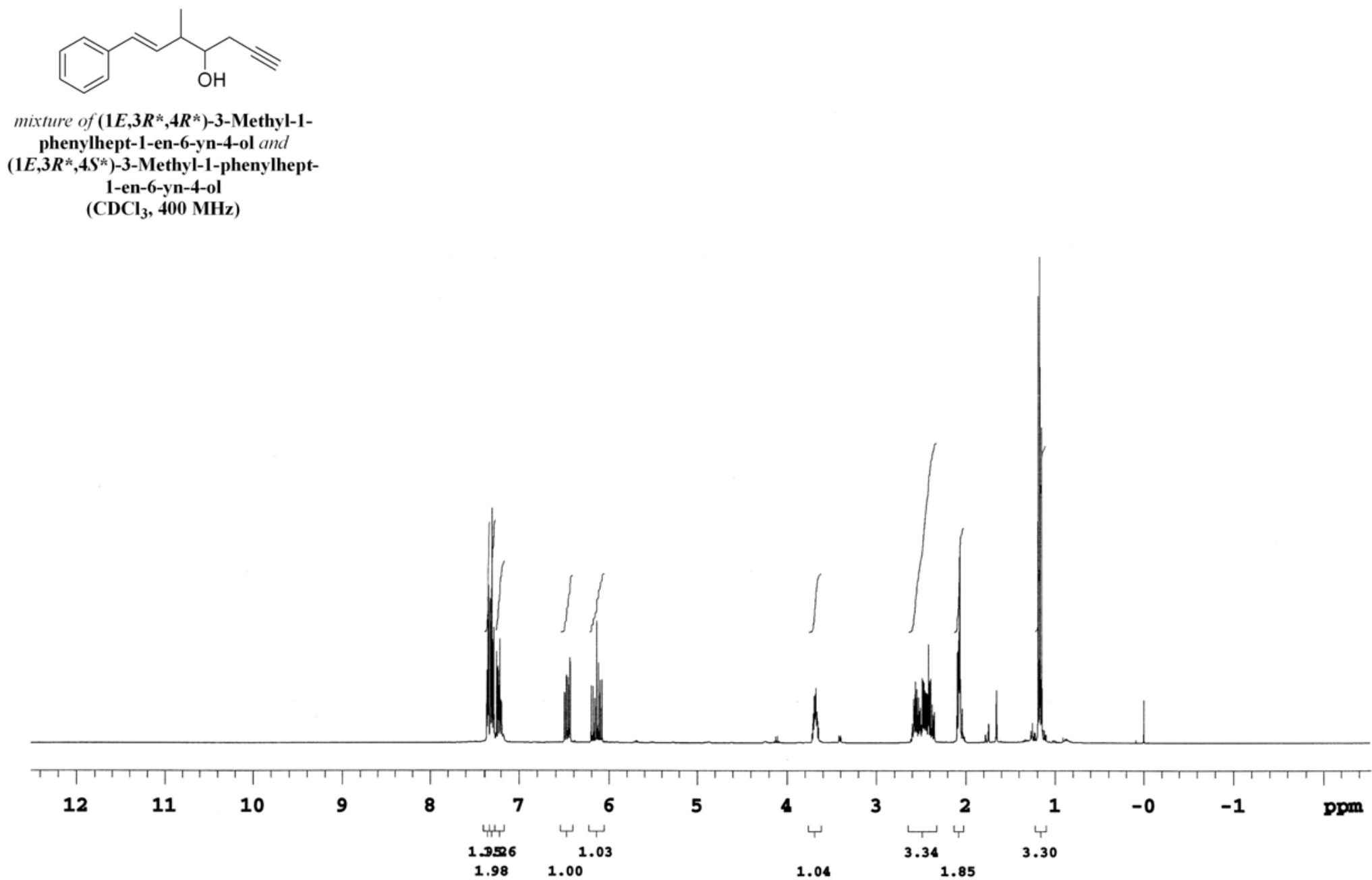




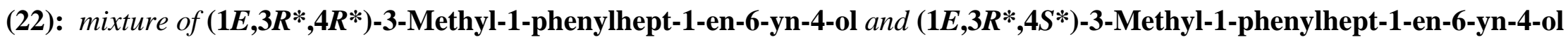

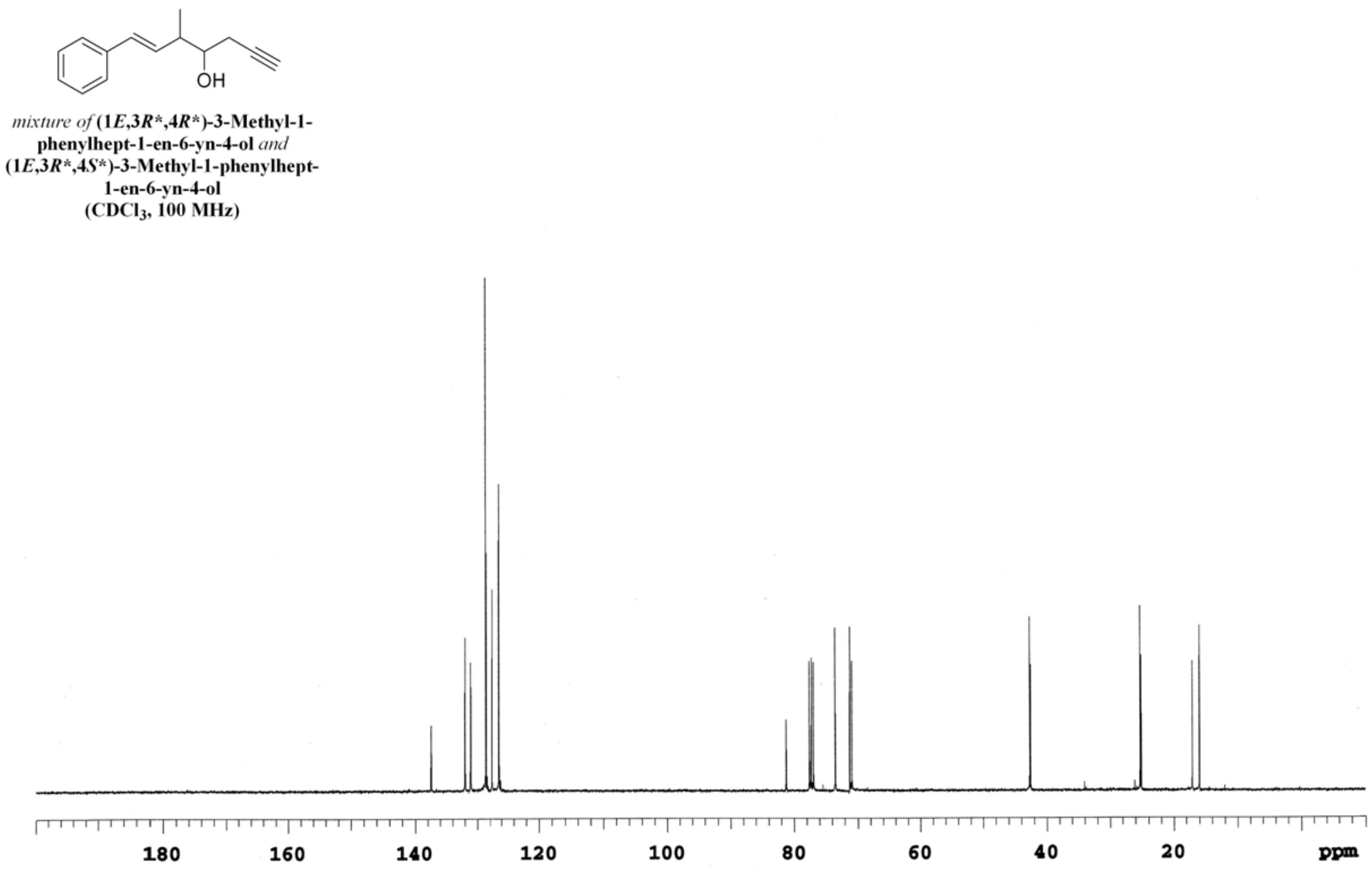


(Sup 5): mixture of (+/-)-(3E)-1-(Benzyloxy)-4-phenylbut-3-en-2-ol and (+/-)-(3Z)-1-(Benzyloxy)-4-phenylbut-3-en-2-ol

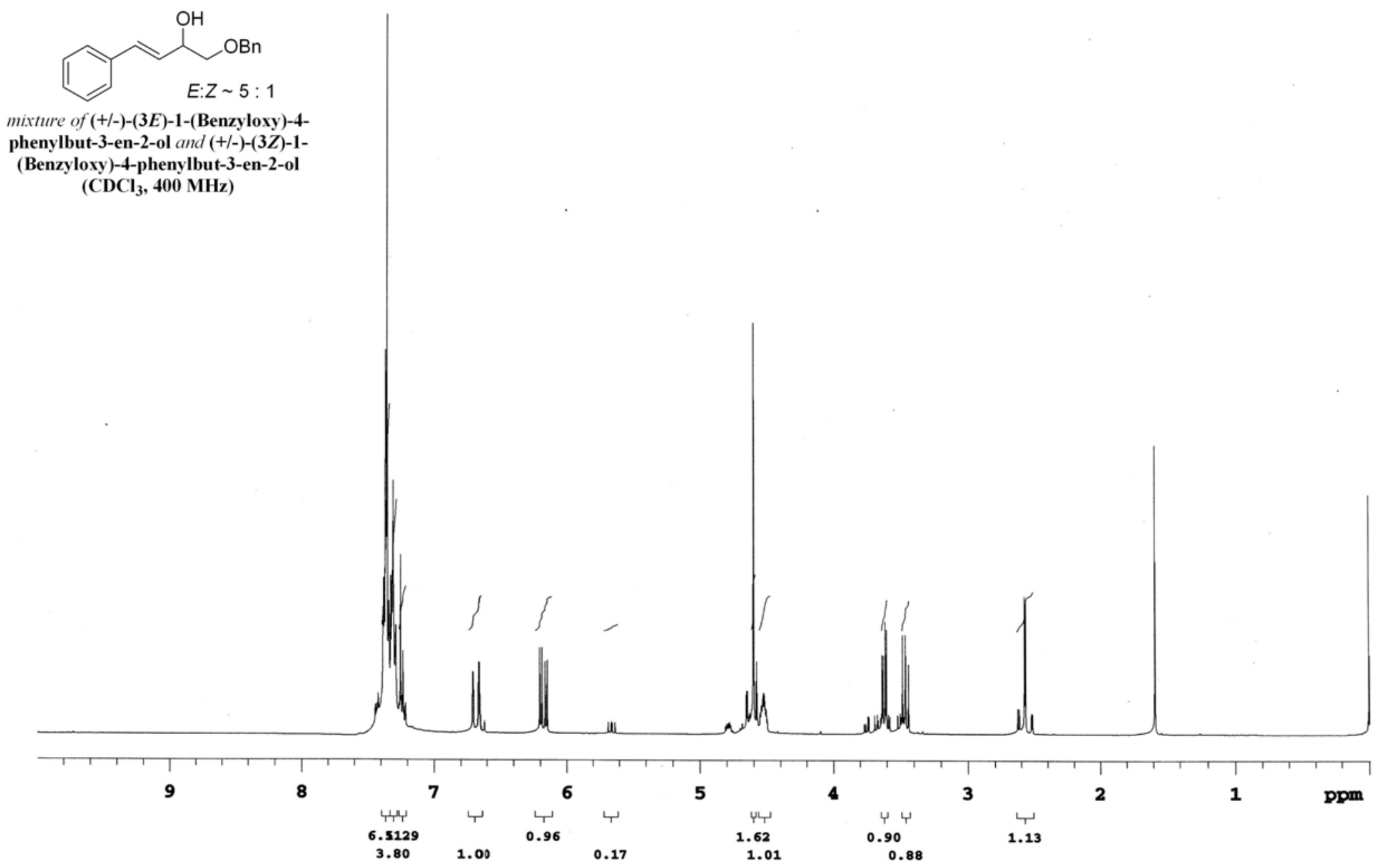


(Sup 5): mixture of (+/-)-(3E)-1-(Benzyloxy)-4-phenylbut-3-en-2-ol and (+/-)-(3Z)-1-(Benzyloxy)-4-phenylbut-3-en-2-ol
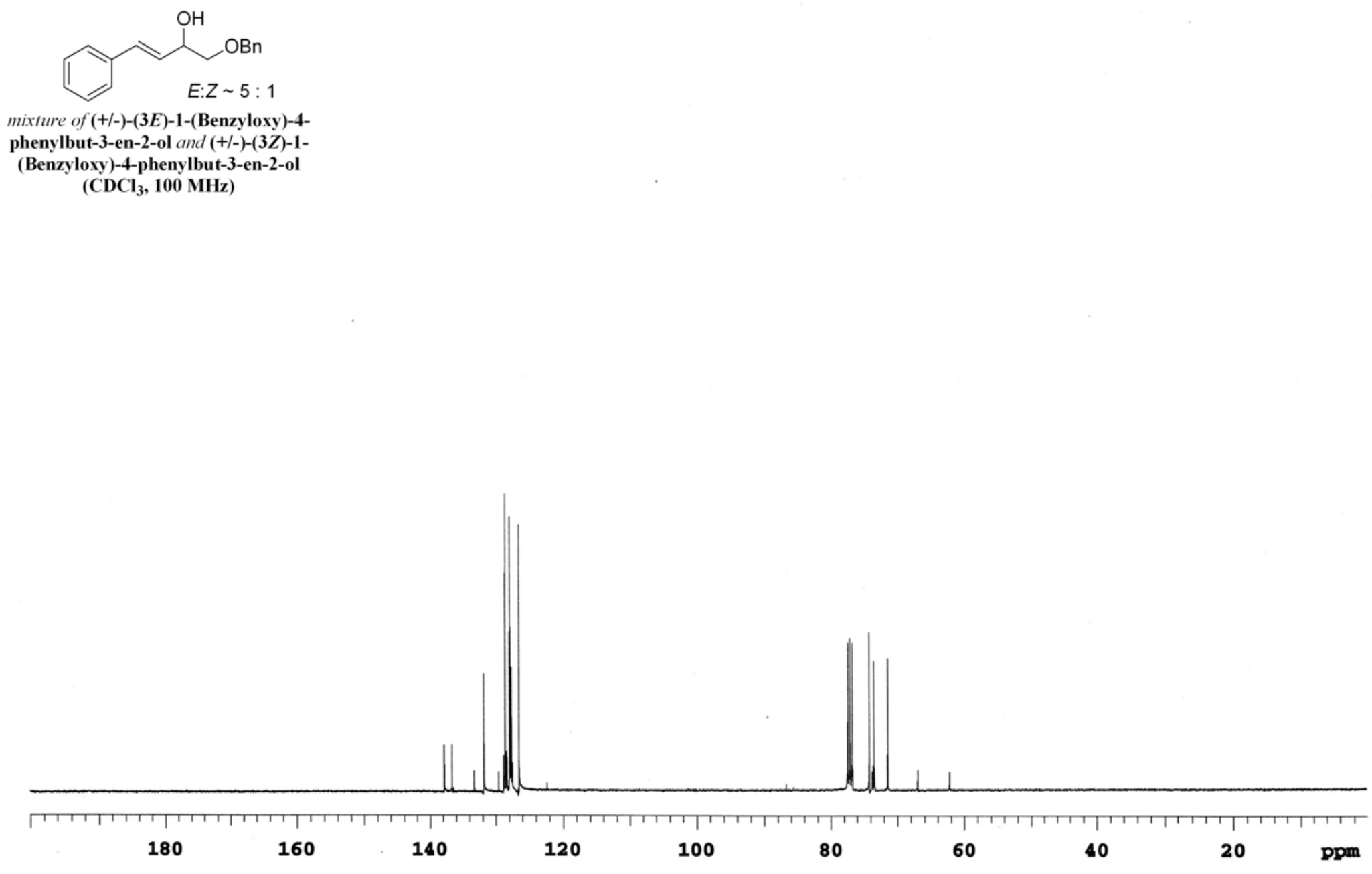


\section{(Sup 6): mixture of (3E)-1-(Benzyloxy)-4-phenylbut-3-en-2-one and (3Z)-1-(Benzyloxy)-4-phenylbut-3-en-2-one}

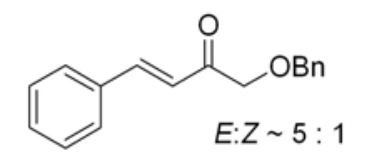

mixture of (3E)-1-(Benzyloxy)-4-

phenylbut-3-en-2-one and (3Z)-1-

(Benzyloxy)-4-phenylbut-3-en-2-one $\left(\mathrm{CDCl}_{3}, 400 \mathrm{MHz}\right)$

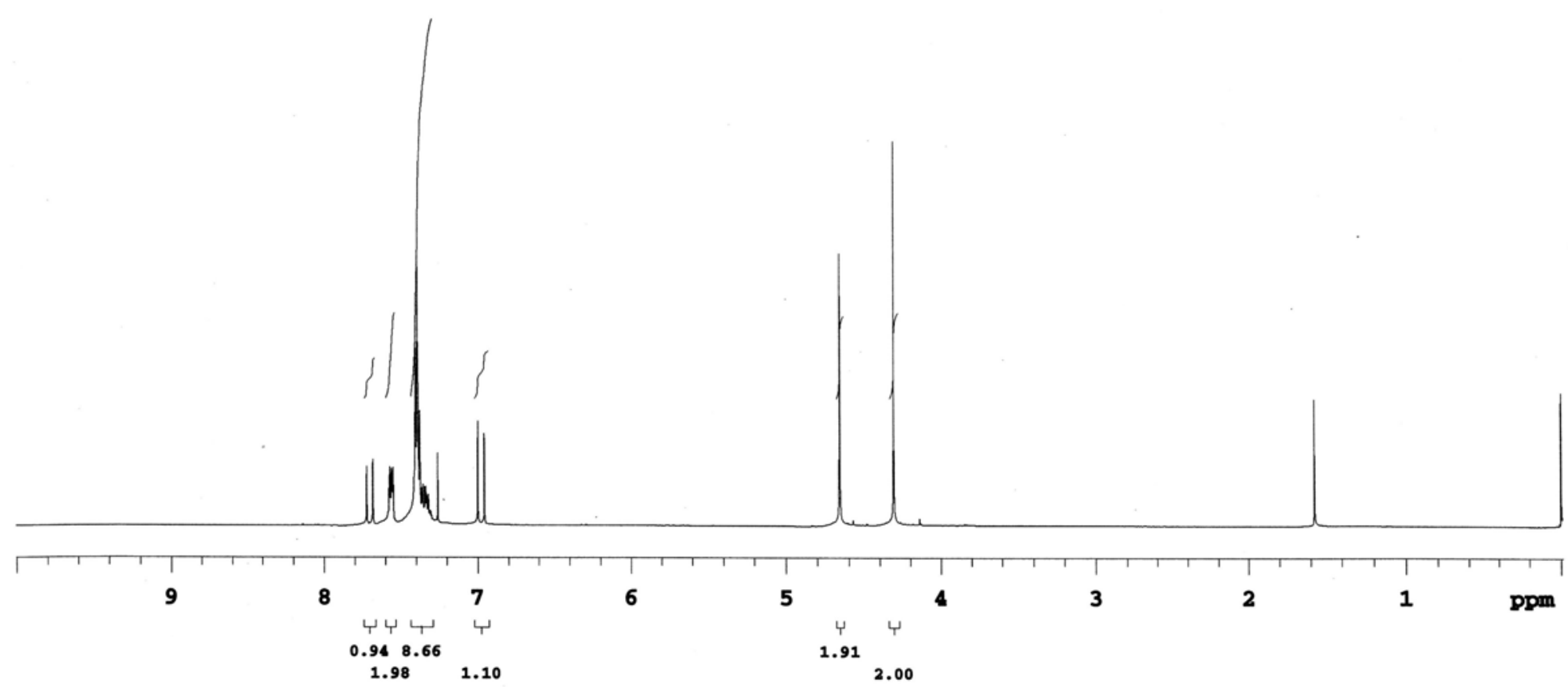


(Sup 6): mixture of (3E)-1-(Benzyloxy)-4-phenylbut-3-en-2-one and (3Z)-1-(Benzyloxy)-4-phenylbut-3-en-2-one

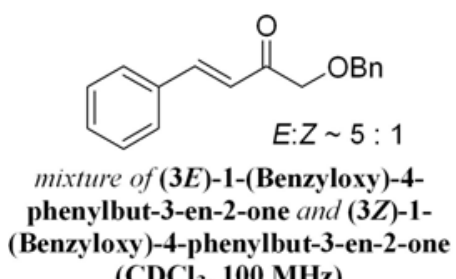

(Benzyloxy)-4-phenylbut-3-en-2-one

$\left(\mathrm{CDCl}_{3}, 100 \mathrm{MHz}\right)$

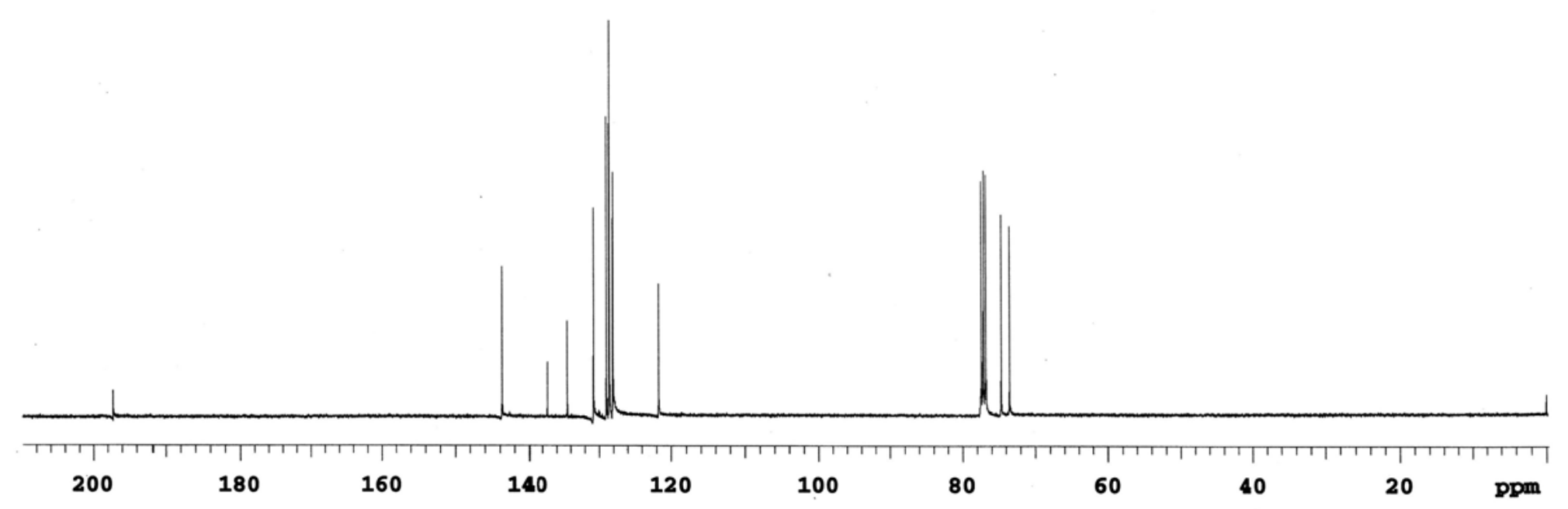




\section{(Sup 7): (+/-)-(3E)-1-(Benzyloxy)-2-(chloromethyl)-4-phenylbut-3-en-2-ol}

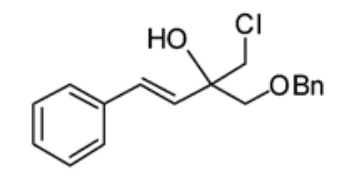

(+/-)-(3E)-1-(Benzyloxy)-2

(chloromethyl)-4-phenylbut-3-en-2-ol

$\left(\mathrm{CDCl}_{3}, 400 \mathrm{MHz}\right)$

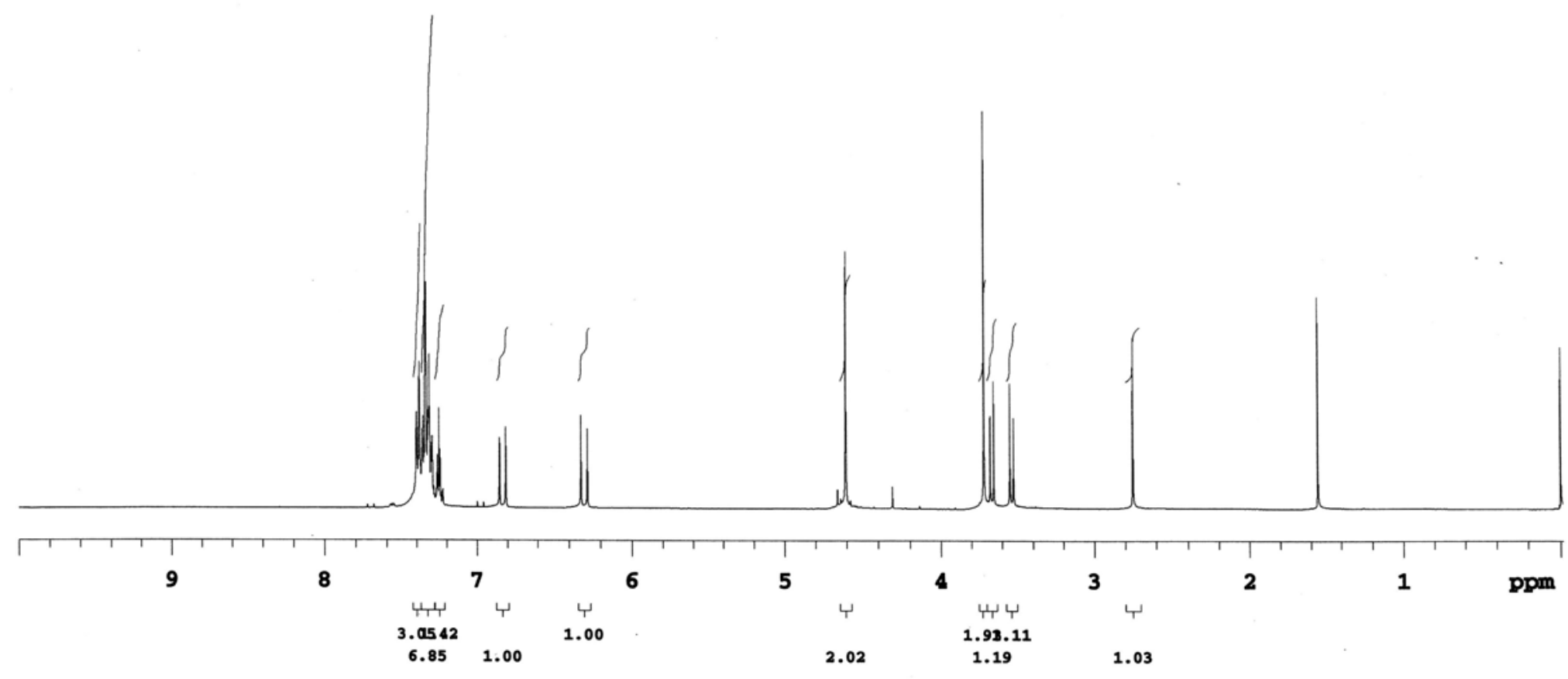


(Sup 7): (+/-)-(3E)-1-(Benzyloxy)-2-(chloromethyl)-4-phenylbut-3-en-2-ol

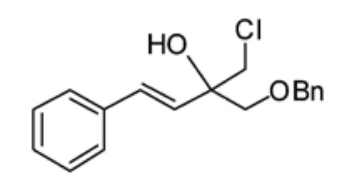

$(+/-)-(3 E)-1-($ Benzyloxy $)-2-$

(chloromethyl)-4-phenylbut-3-en-2-ol

$\left(\mathrm{CDCl}_{3}, 100 \mathrm{MHz}\right)$

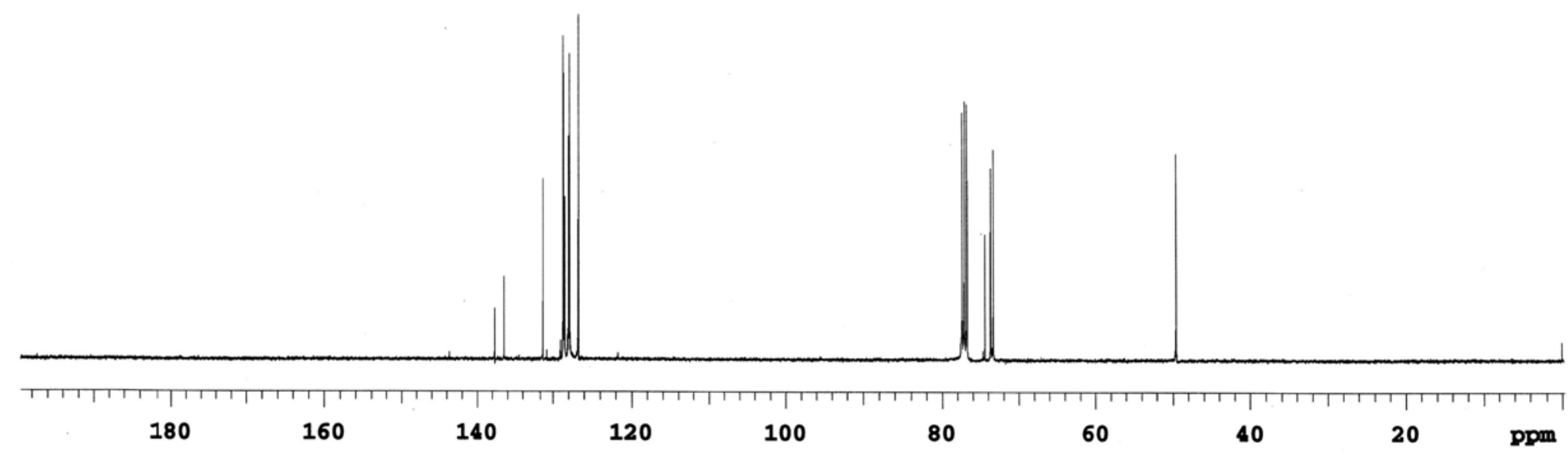


(Sup 8): (+/-)-(3Z)-1-(Benzyloxy)-2-(chloromethyl)-4-phenylbut-3-en-2-ol

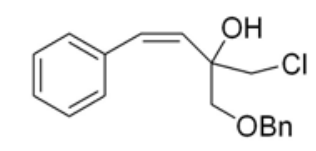

(+/-)-(3Z)-1-(Benzyloxy)-2-

(chloromethyl)-4-phenylbut-3-en-2-ol

$\left(\mathrm{CDCl}_{3}, 400 \mathrm{MHz}\right)$

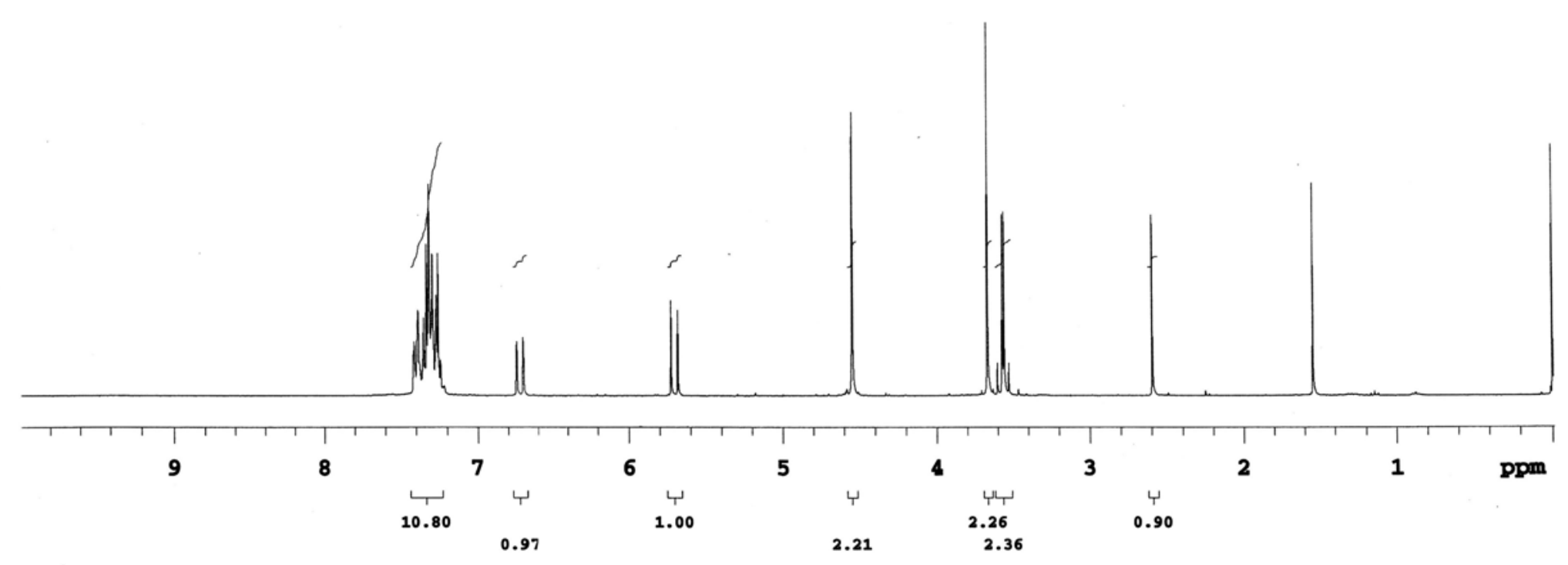


(Sup 8): (+/-)-(3Z)-1-(Benzyloxy)-2-(chloromethyl)-4-phenylbut-3-en-2-ol

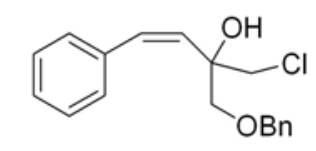

(+/-)-(3Z)-1-(Benzyloxy)-2-

(chloromethyl)-4-phenylbut-3-en-2-ol

$\left(\mathrm{CDCl}_{3}, 100 \mathrm{MHz}\right)$

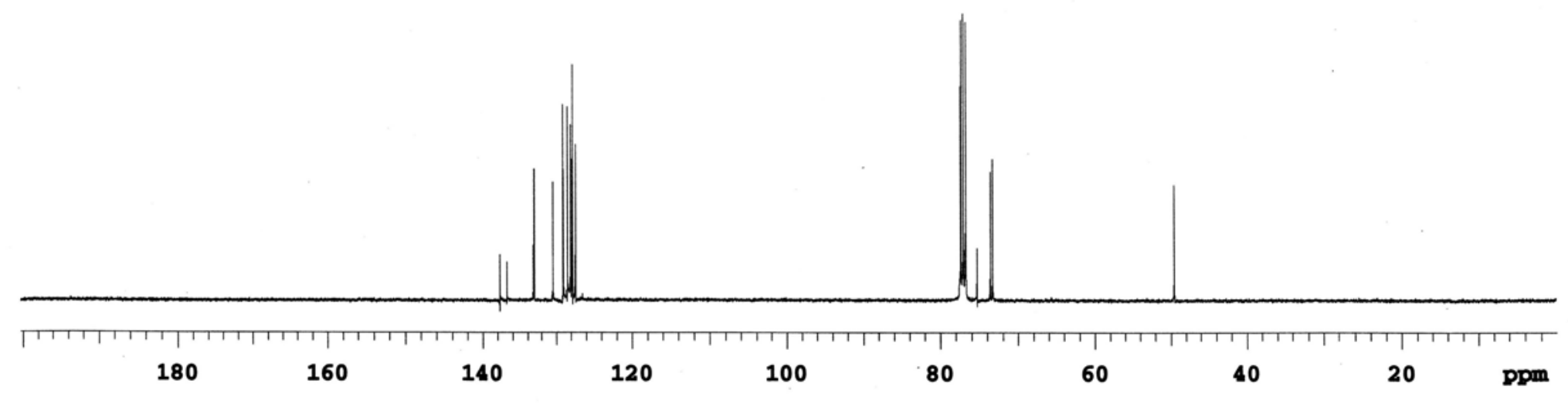


(23): (+/-)-2-[(Benzyloxy)methyl]-2-[(E)-2-phenylvinyl]oxirane

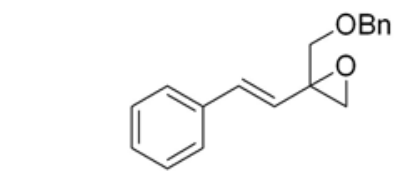

$(+/-)-2-[($ Benzyloxy $)$ methyl $]-2-[(E)-2-$

phenylvinyl]oxirane

$\left(\mathrm{CDCl}_{3}, 400 \mathrm{MHz}\right)$

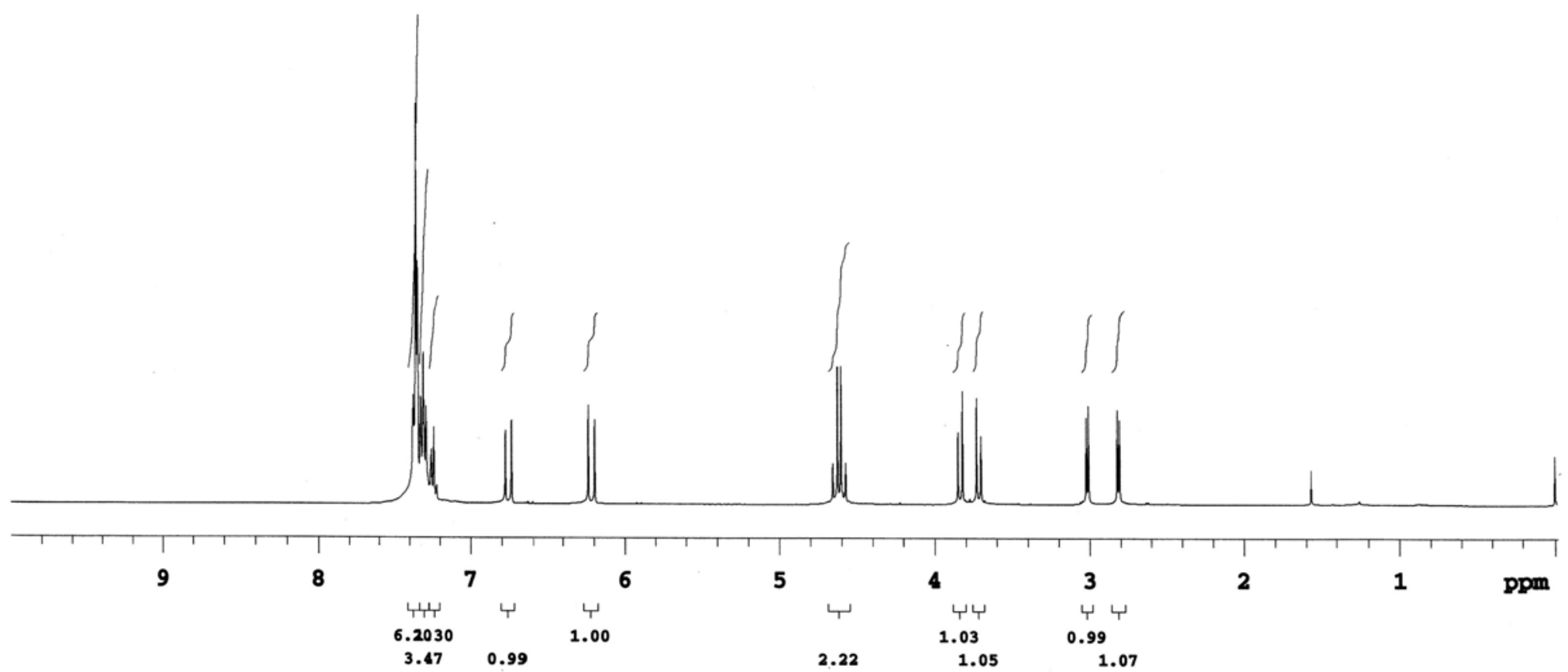


(23): (+/-)-2-[(Benzyloxy)methyl]-2-[(E)-2-phenylvinyl]oxirane

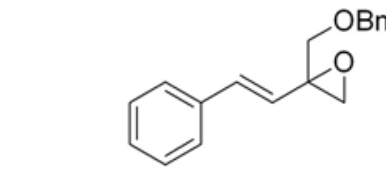

$(+/-)-2-[($ Benzyloxy $)$ methyl]-2- $[(E)-2-$

phenylvinyl oxirane

$\left(\mathrm{CDCl}_{3}, 100 \mathrm{MHz}\right)$

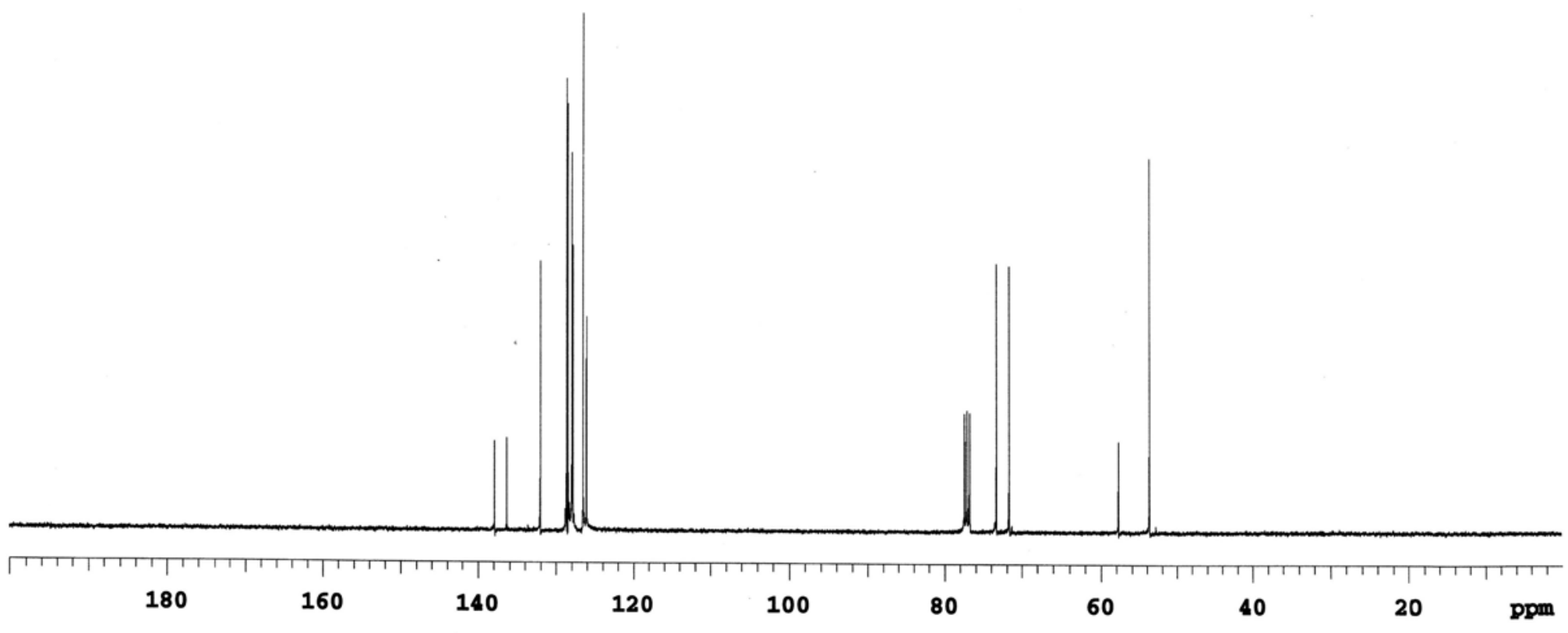


(24): mixture of $\left(1 E, 3 S^{*}, 4 R^{*}\right)-3-[(B e n z y l o x y) m e t h y l]-1-p h e n y l h e p t-1-e n-6-y n-4-o l$ and $\left(1 E, 3 S^{*}, 4 S^{*}\right)-3-[(B e n z y l o x y) m e t h y l]-1-$ phenylhept-1-en-6-yn-4-ol
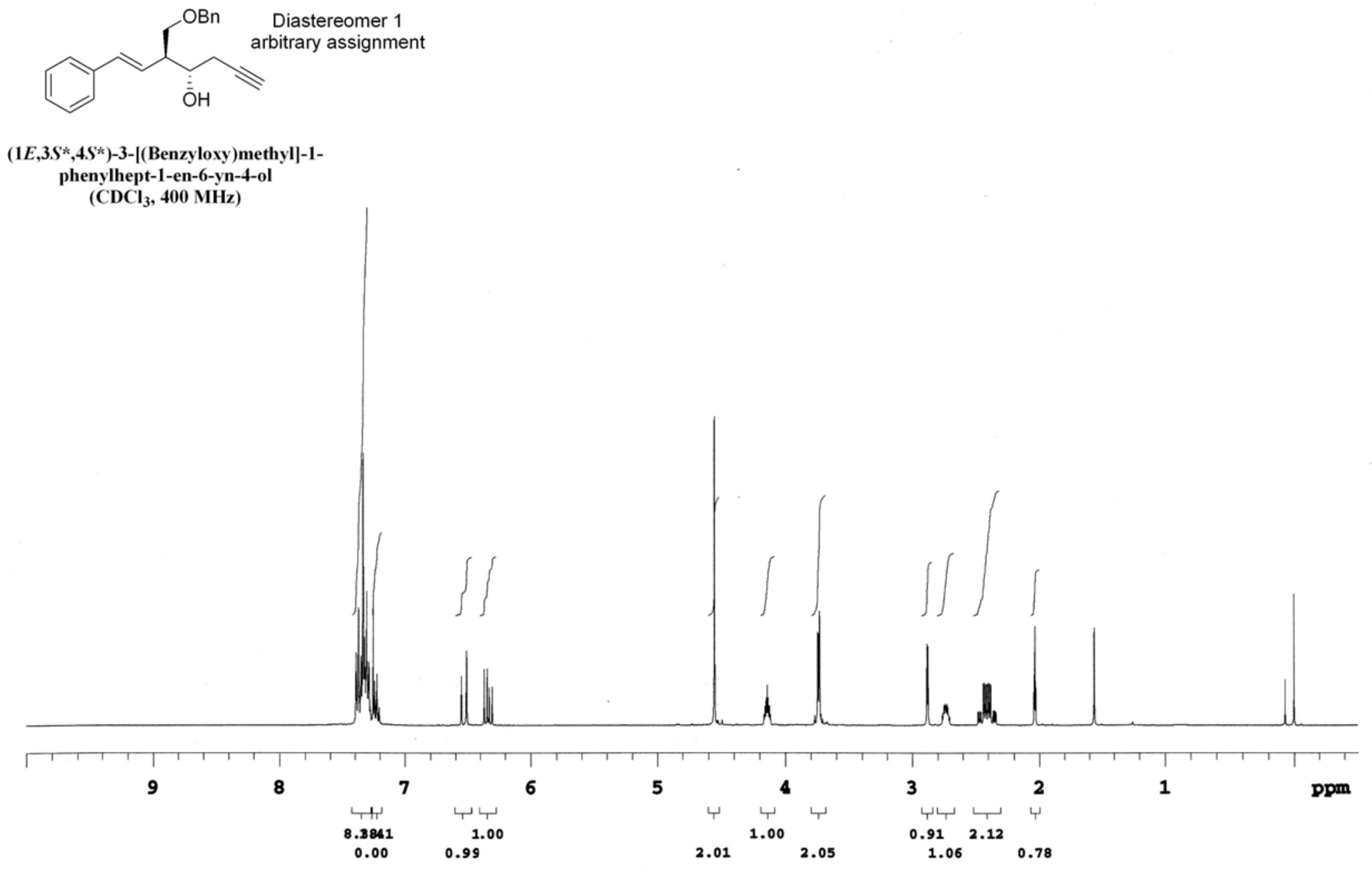
(24): mixture of $\left(1 E, 3 S^{*}, 4 R^{*}\right)-3-[(B e n z y l o x y) m e t h y l]-1-p h e n y l h e p t-1-e n-6-y n-4-o l$ and $\left(1 E, 3 S^{*}, 4 S^{*}\right)-3-[(B e n z y l o x y) m e t h y l]-1-$ phenylhept-1-en-6-yn-4-ol

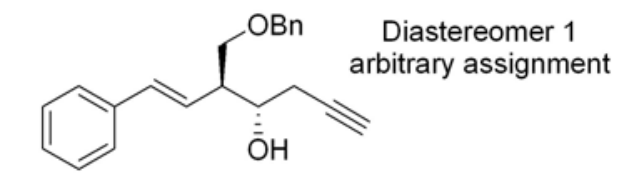

$\left(1 E, 3 S^{*}, 4 S^{\star}\right)-3-[($ Benzyloxy $)$ methyl $]-1-$

phenylhept-1-en-6-yn-4-ol $\left(\mathrm{CDCl}_{3}, 100 \mathrm{MHz}\right)$

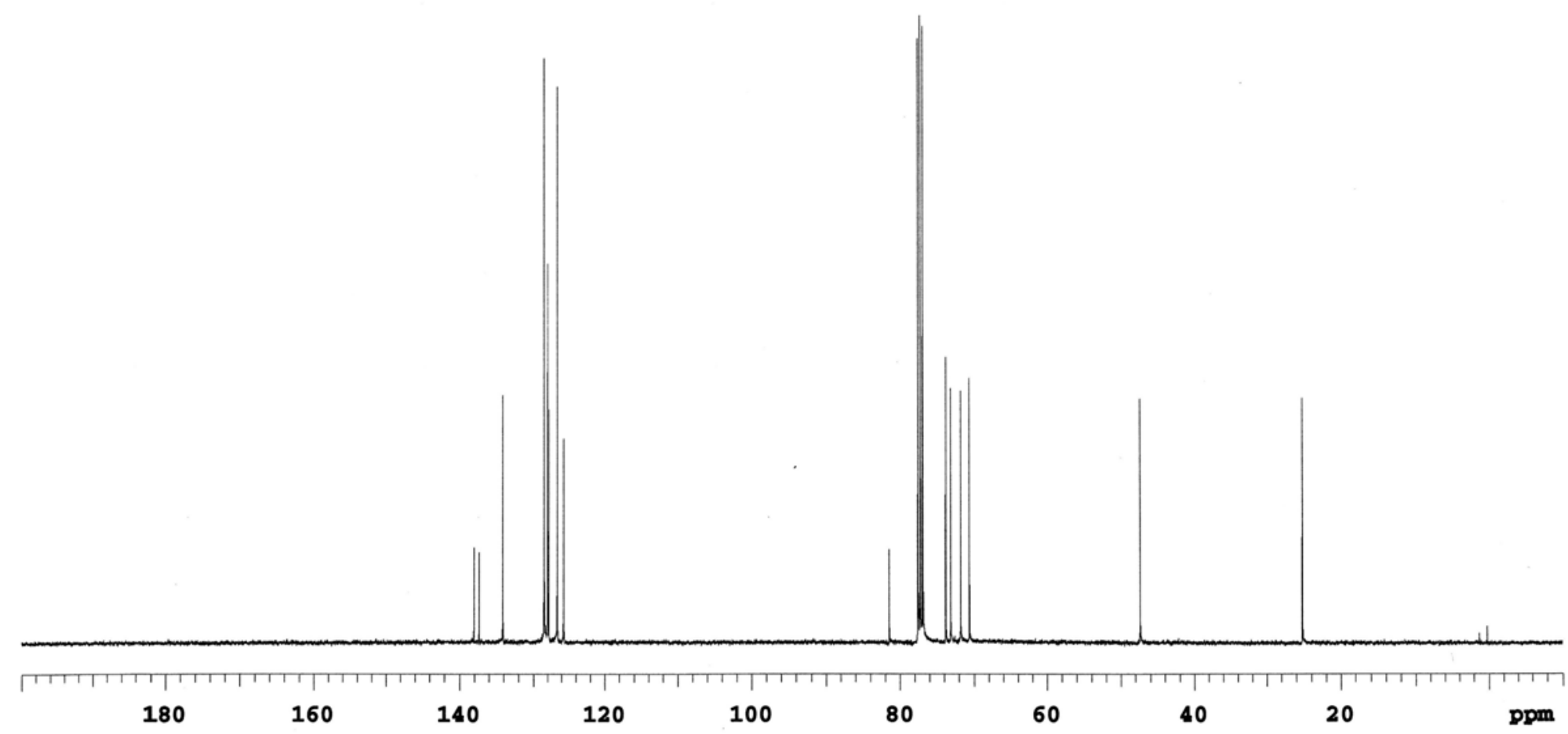


(24): mixture of $\left(1 E, 3 S^{*}, 4 R^{*}\right)-3-[(B e n z y l o x y) m e t h y l]-1-p h e n y l h e p t-1-e n-6-y n-4-o l$ and $\left(1 E, 3 S^{*}, 4 S^{*}\right)-3-[(B e n z y l o x y) m e t h y l]-1-$ phenylhept-1-en-6-yn-4-ol

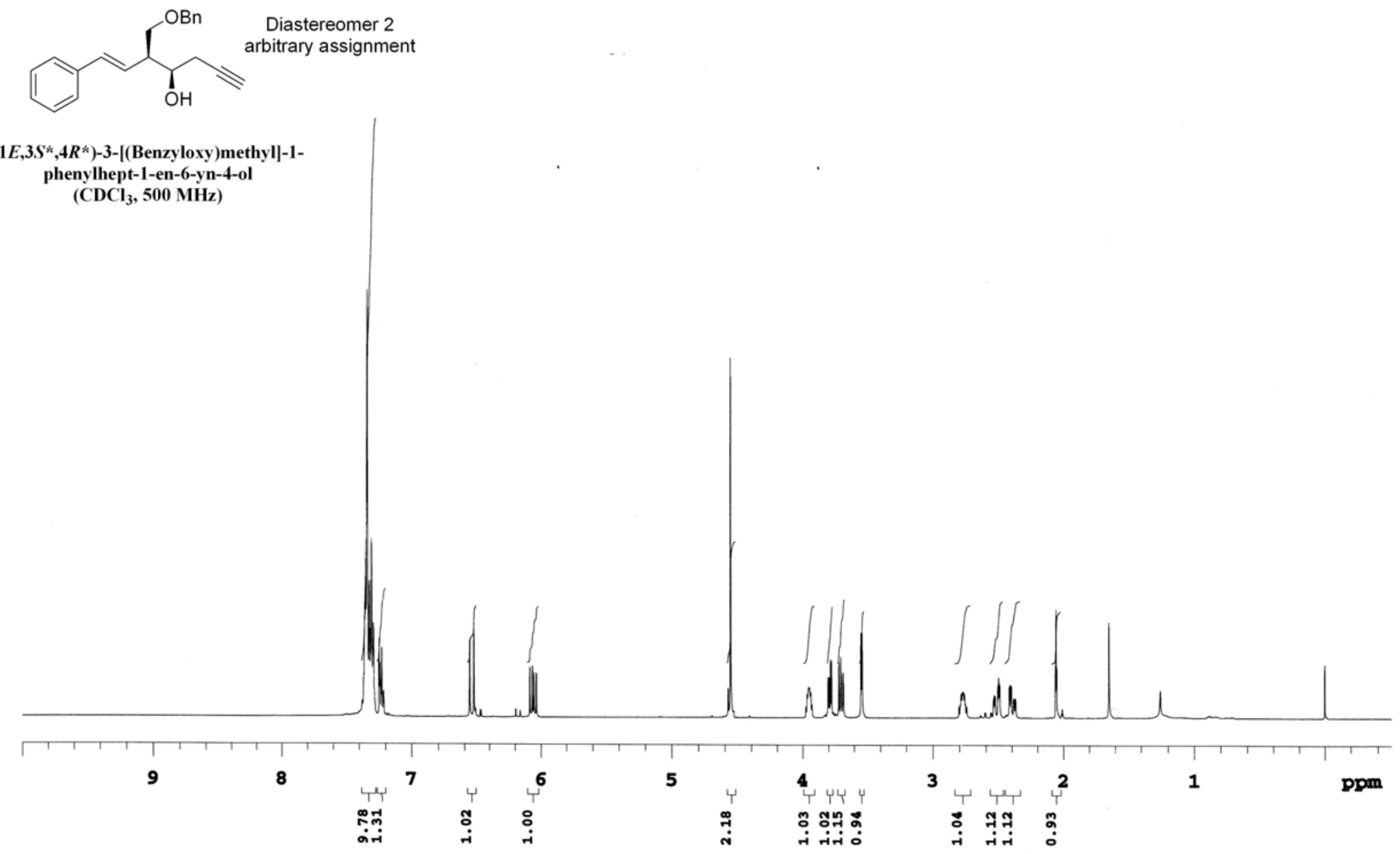


(24): mixture of $\left(1 E, 3 S^{*}, 4 R^{*}\right)-3-[(B e n z y l o x y) m e t h y l]-1-p h e n y l h e p t-1-e n-6-y n-4-o l$ and $\left(1 E, 3 S^{*}, 4 S^{*}\right)-3-[(B e n z y l o x y) m e t h y l]-1-$ phenylhept-1-en-6-yn-4-ol

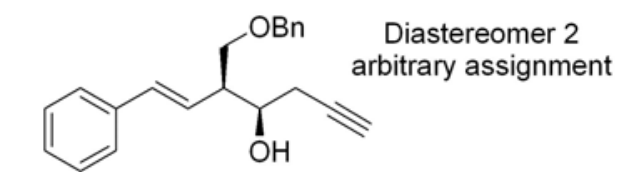

$\left(1 E, 3 S^{\star}, 4 R^{\star}\right)-3-[($ Benzyloxy $)$ methyl $]-1-$

phenylhept-1-en-6-yn-4-ol $\left(\mathrm{CDCl}_{3}, 125 \mathrm{MHz}\right)$

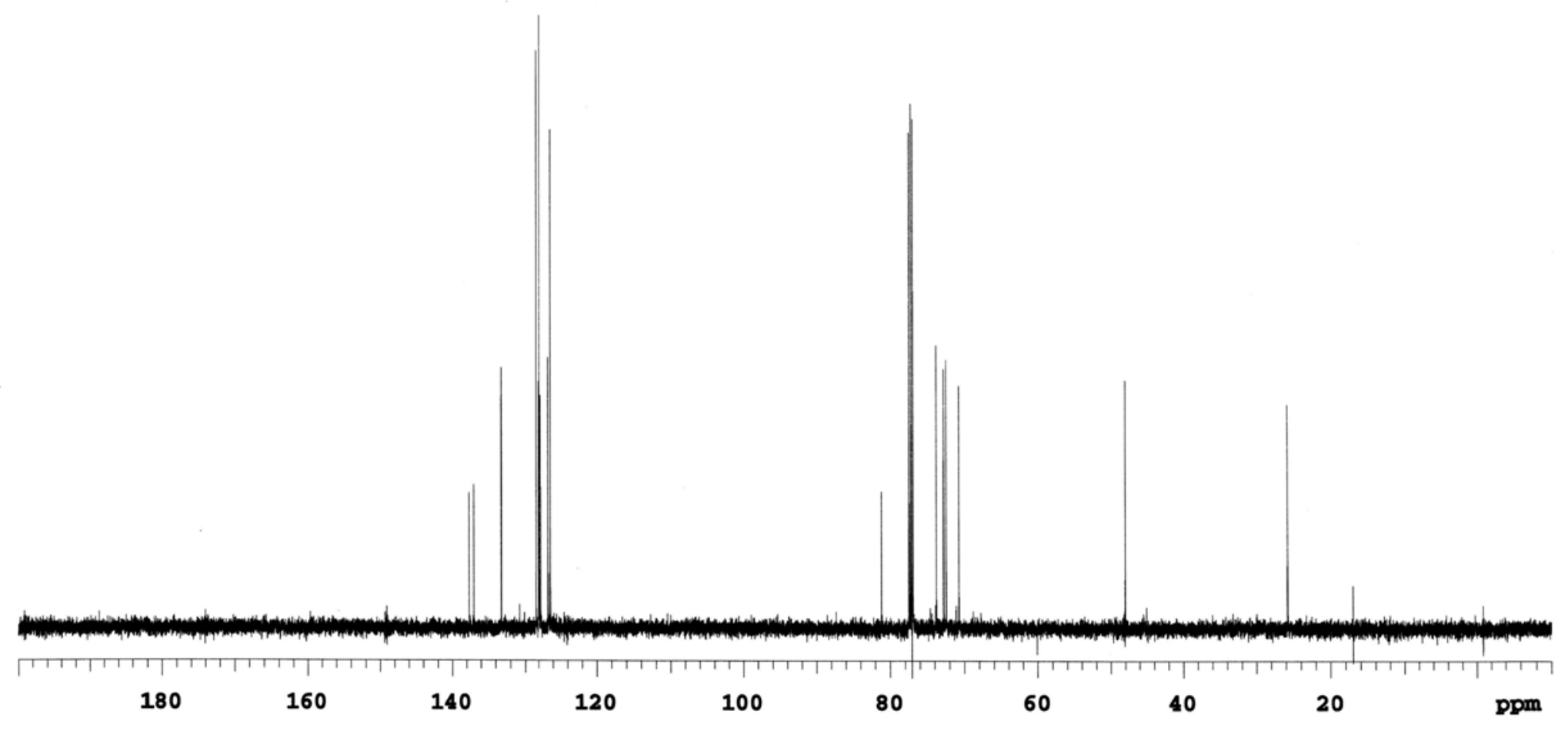


(26): (+/-)-(1E)-1-Phenylhept-1-en-6-yn-4-ol

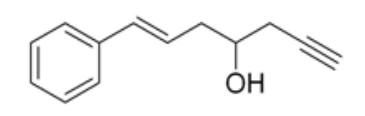

(+/-)-(1E)-1-Phenylhept-1-en-6-yn-4-ol $\left(\mathrm{CDCl}_{3}, 500 \mathrm{MHz}\right)$

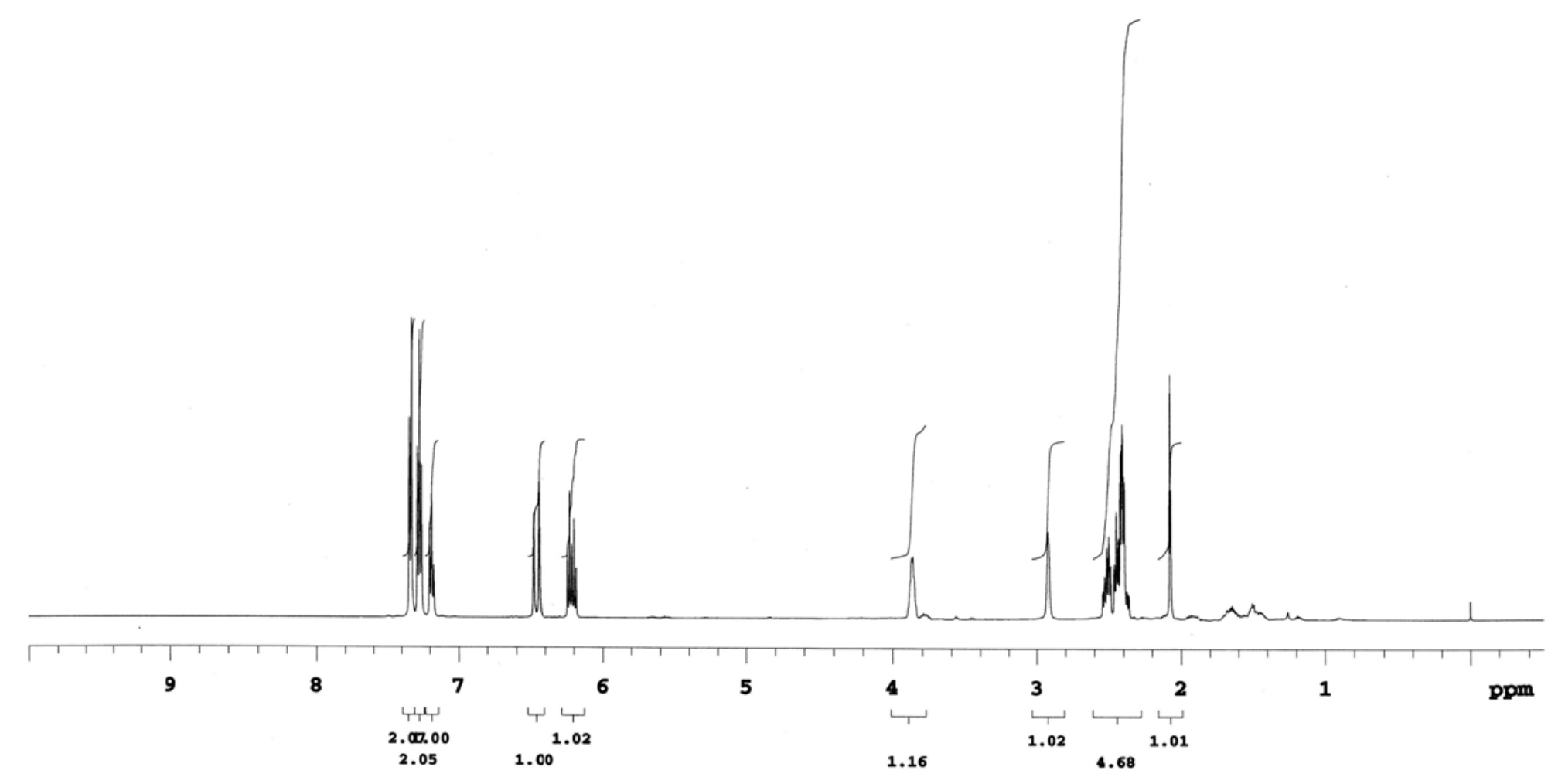


(26): (+/-)-(1E)-1-Phenylhept-1-en-6-yn-4-ol

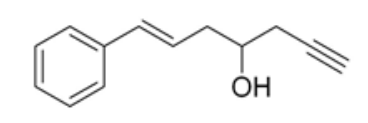

(+/-)-(1E)-1-Phenylhept-1-en-6-yn-4-ol $\left(\mathrm{CDCl}_{3}, 125 \mathrm{MHz}\right)$

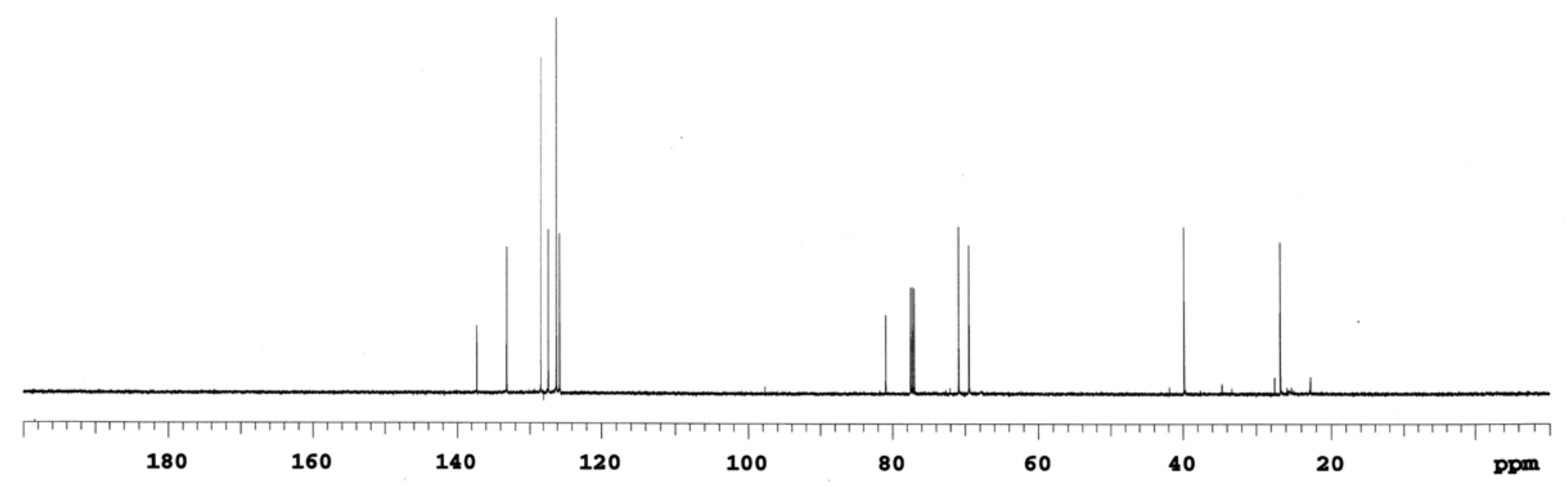


(28): (+/-)-Hept-1-en-6-yn-4-ol

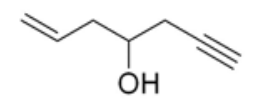

(+/-)-Hept-1-en-6-yn-4-ol

$\left(\mathrm{CDCl}_{3}, 400 \mathrm{MHz}\right)$

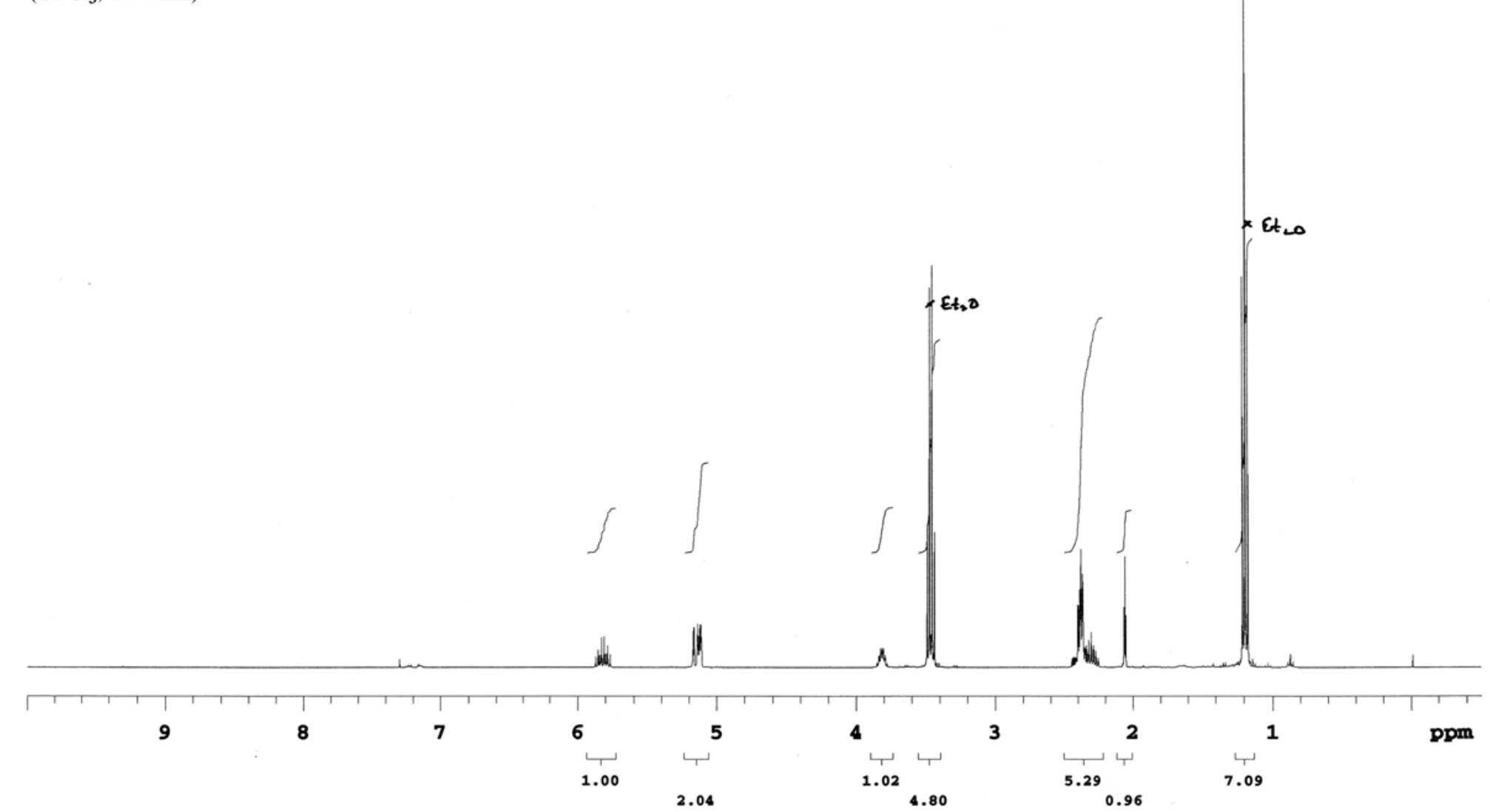


(28): (+/-)-Hept-1-en-6-yn-4-ol

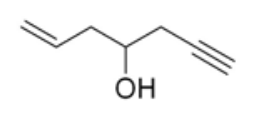

(+/-)-Hept-1-en-6-yn-4-ol $\left(\mathrm{CDCl}_{3}, 400 \mathrm{MHz}\right)$

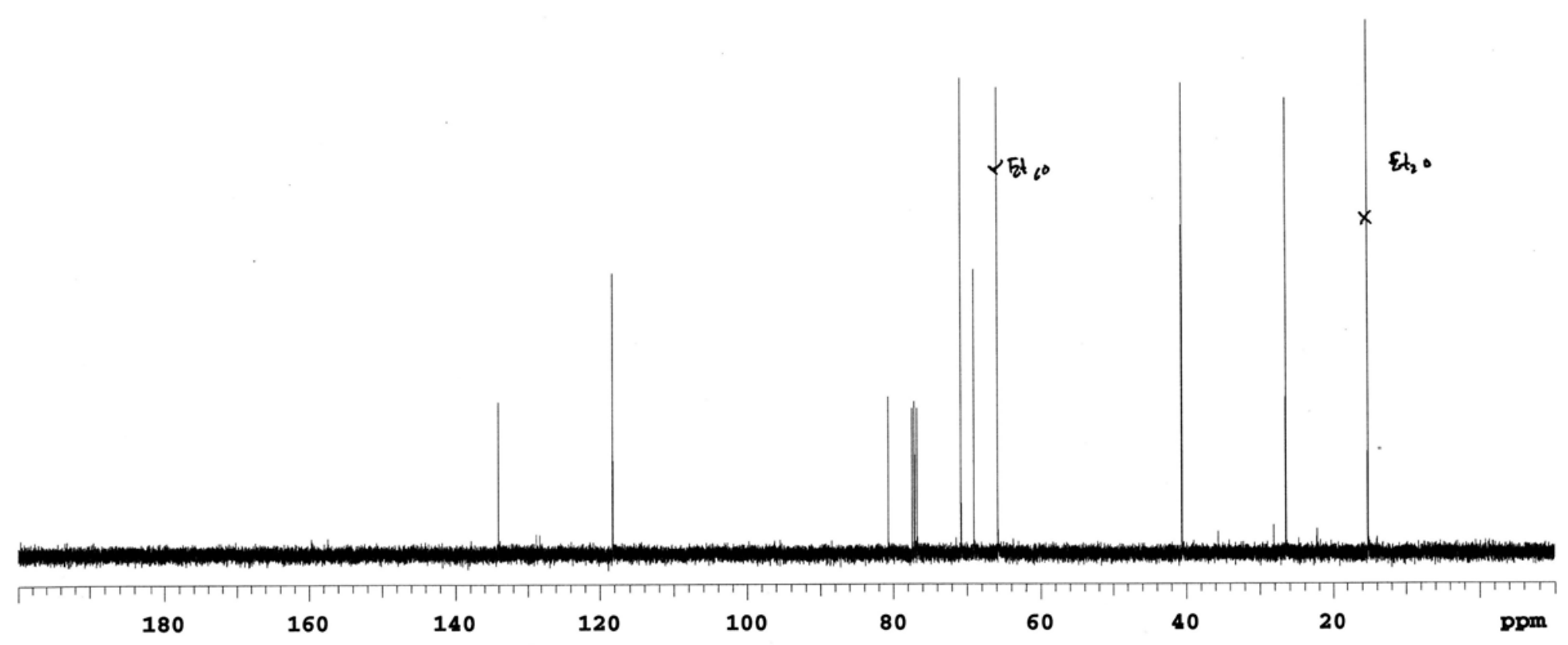


(Sup 9): (+/-)-tert-Butyl(dimethyl)\{[(3E)-1-prop-2-ynyloct-3-enyl]oxy\}silane

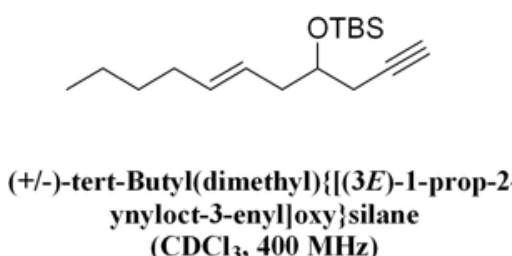

ynyloct-3-enyl|oxy\}silane

$\left(\mathrm{CDCl}_{3}, 400 \mathrm{MHz}\right)$ 
(Sup 9): (+/-)-tert-Butyl(dimethyl)\{[(3E)-1-prop-2-ynyloct-3-enyl]oxy\}silane

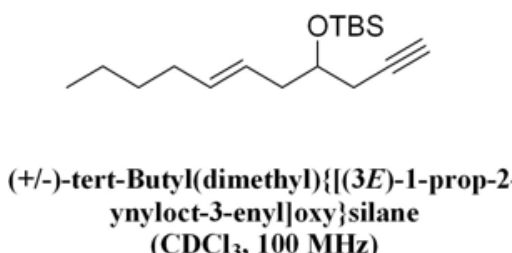

(CDCl, $100 \mathrm{MHz})$
(

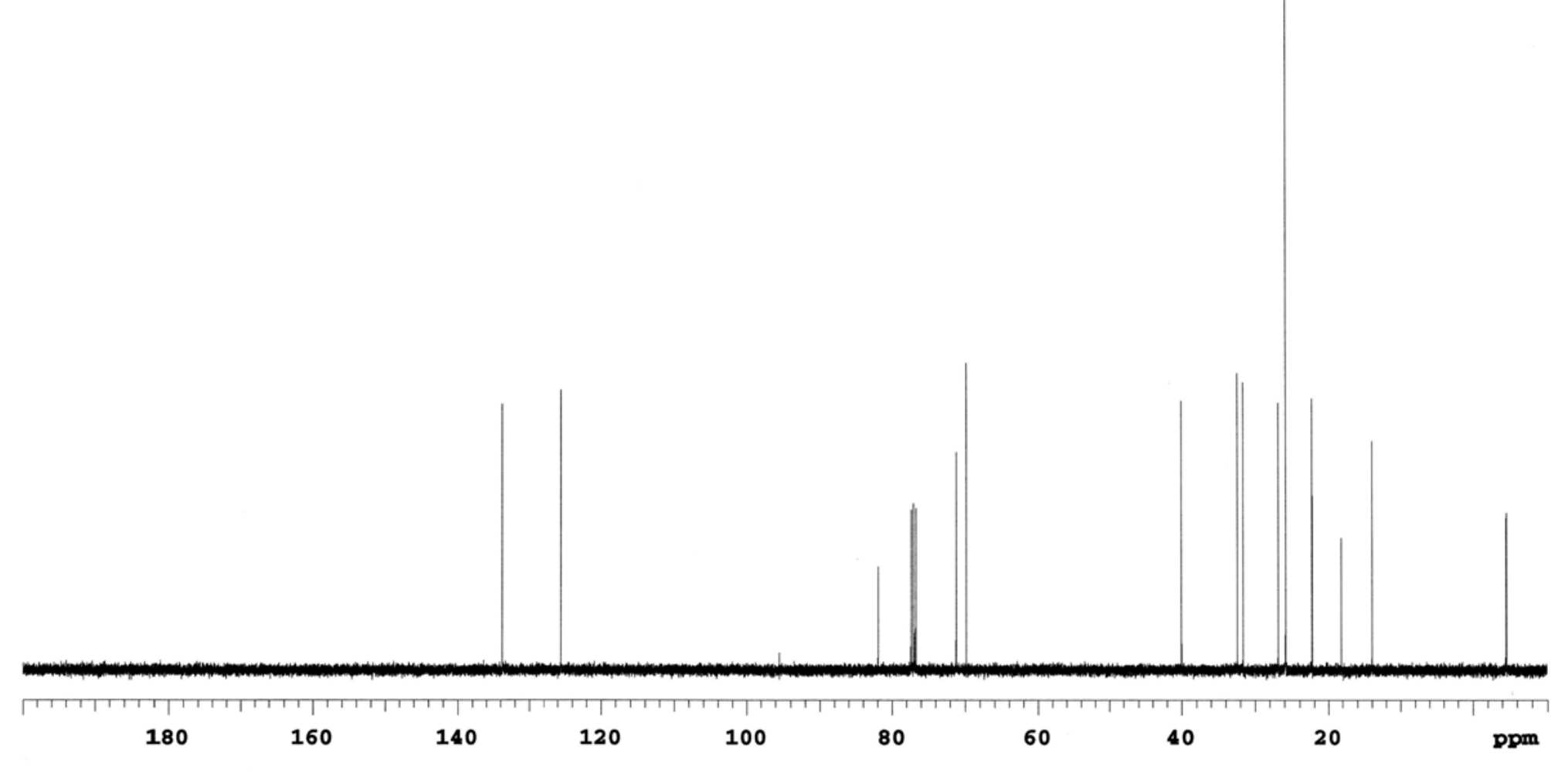


(30): (+/-)-(6E)-Undec-6-en-1-yn-4-ol

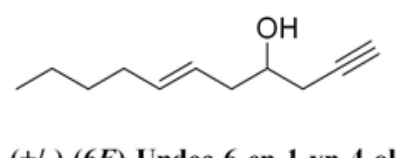

(+/-)-(6E)-Undec-6-en-1-yn-4-ol $\left(\mathrm{CDCl}_{3}, 300 \mathrm{MHz}\right)$

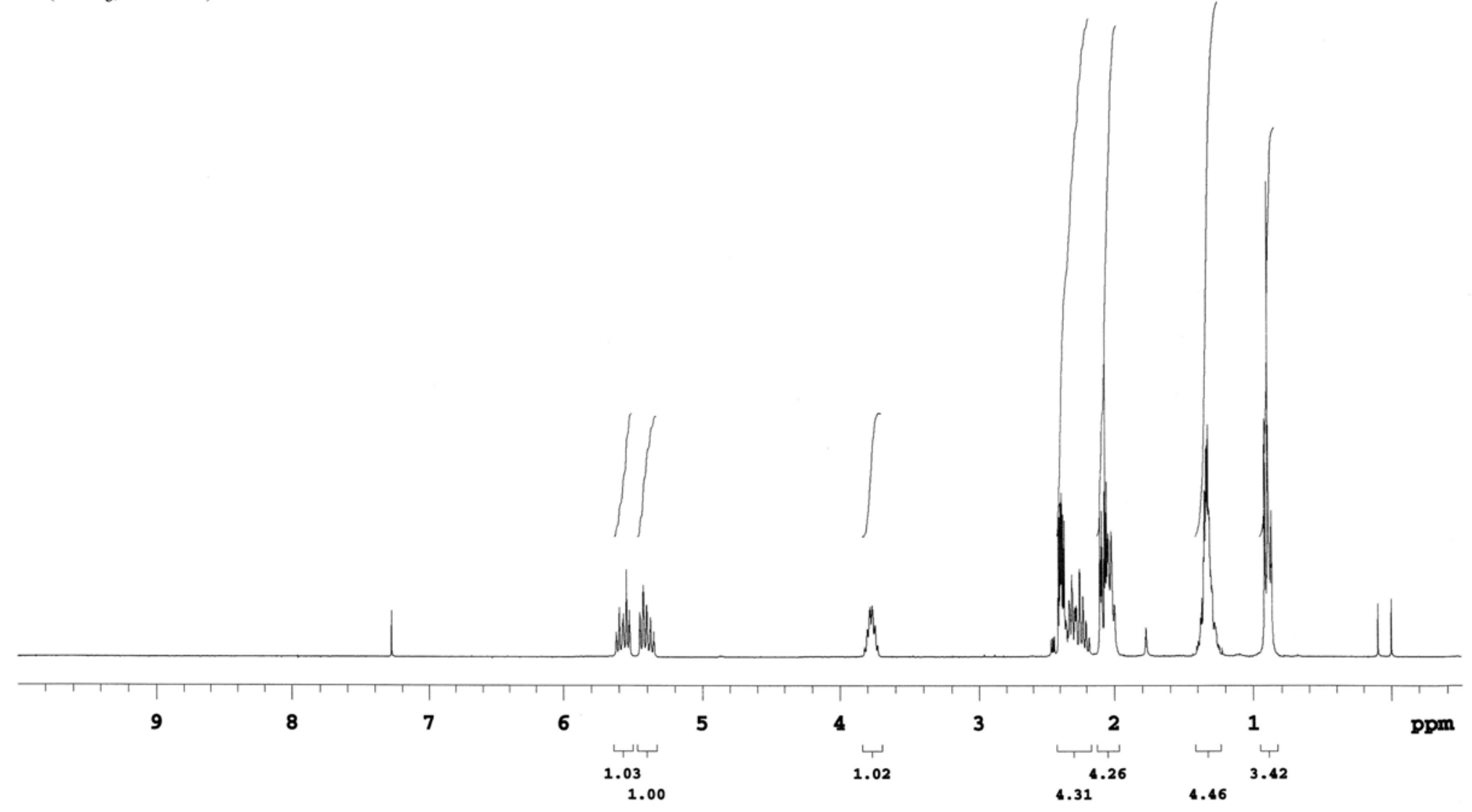


(30): (+/-)-(6E)-Undec-6-en-1-yn-4-ol

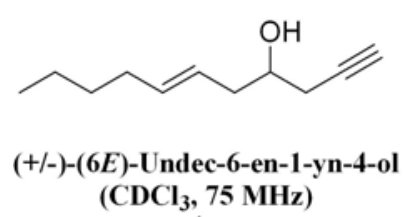

$\left(\mathrm{CDCl}_{3}, 75 \mathrm{MHz}\right)$

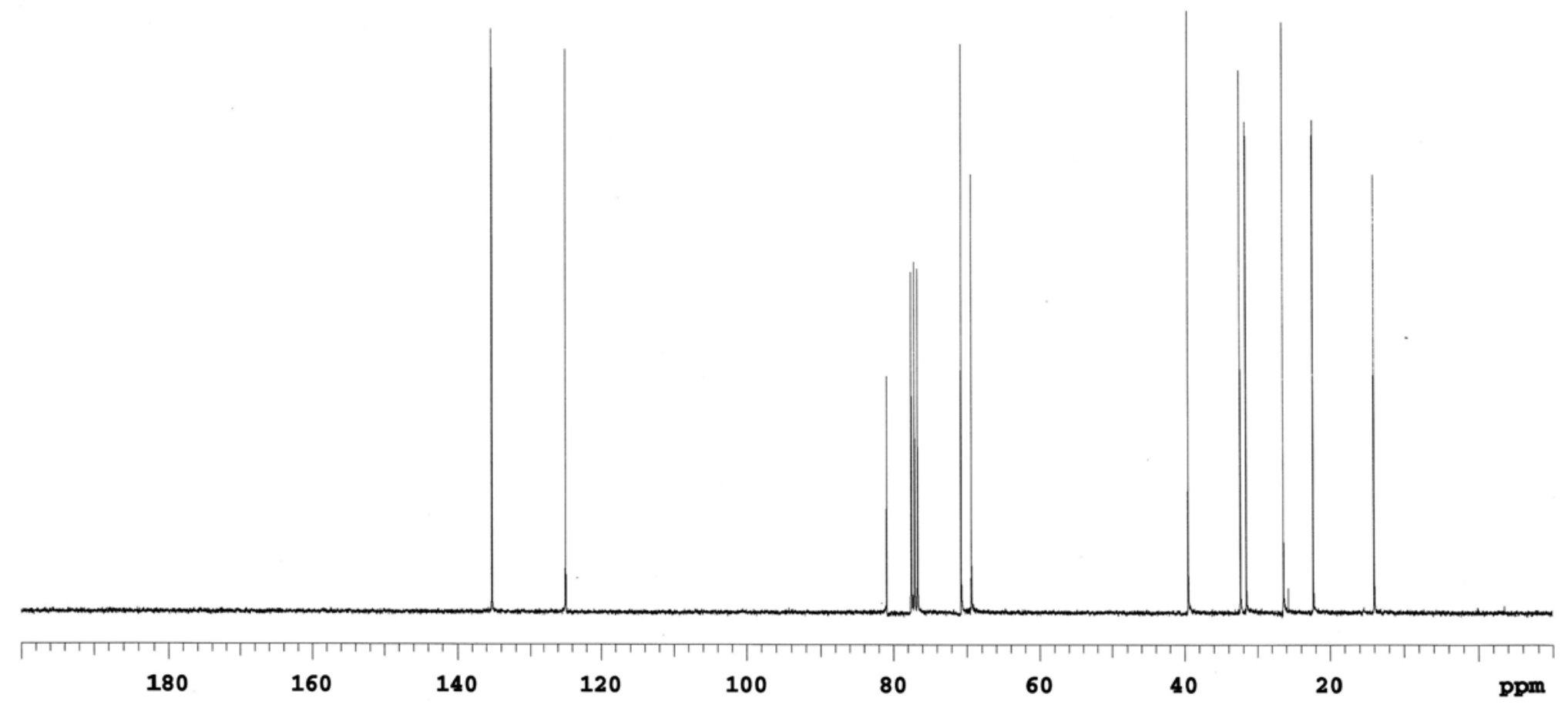




\section{(Sup 10): (+/-)-tert-Butyl\{[1-(cyclohex-1-en-1-ylmethyl)but-3-ynyl]oxy\} dimethyl silane}

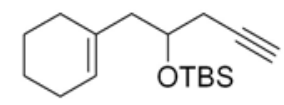

(+/-)-tert-Butyl\{[1-(cyclohex-1-en-1-

ylmethyl)but-3-ynyl]oxy\}dimethylsilane

$$
\left(\mathrm{CDCl}_{3}, 400 \mathrm{MHz}\right)
$$


(Sup 10): (+/-)-tert-Butyl\{[1-(cyclohex-1-en-1-ylmethyl)but-3-ynyl]oxy\} dimethyl silane

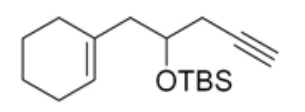

(+/-)-tert-Butyl\{[1-(cyclohex-1-en-1-

methyl)but-3-ynyl] oxy \}dimethylsilane

$\left(\mathrm{CDCl}_{3}, 100 \mathrm{MHz}\right)$

$$
\left(\mathrm{CDCl}_{3}, 100 \mathrm{MHz}\right)
$$

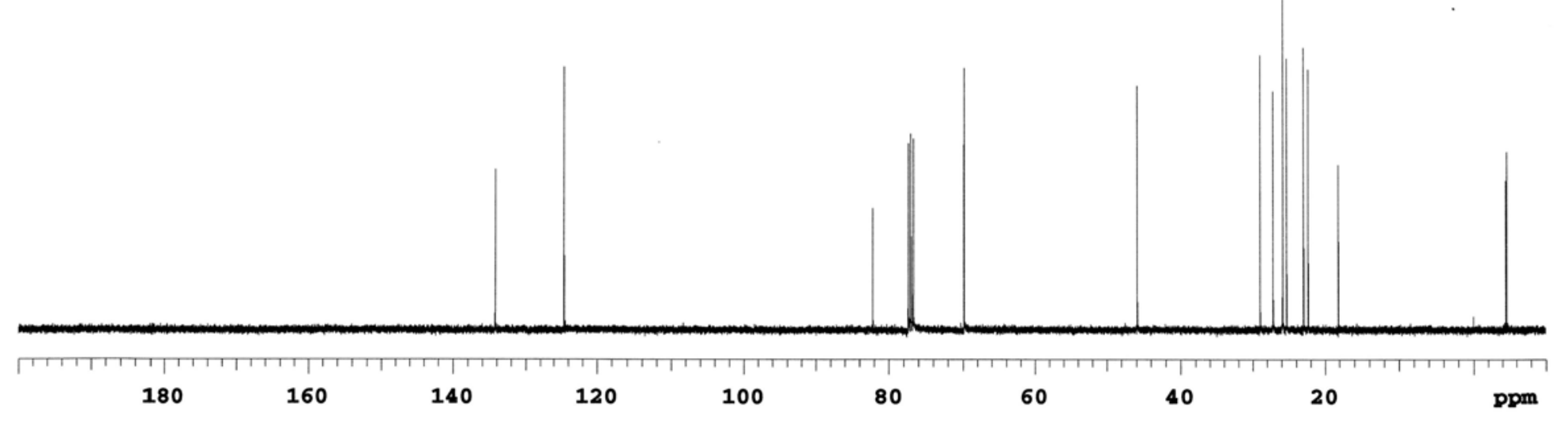


(32): (+/-)-1-Cyclohex-1-en-1-ylpent-4-yn-2-ol

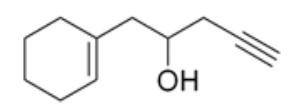

(+/-)-1-Cyclohex-1-en-1-ylpent-4-yn-2-ol $\left(\mathrm{CDCl}_{3}, 300 \mathrm{MHz}\right)$

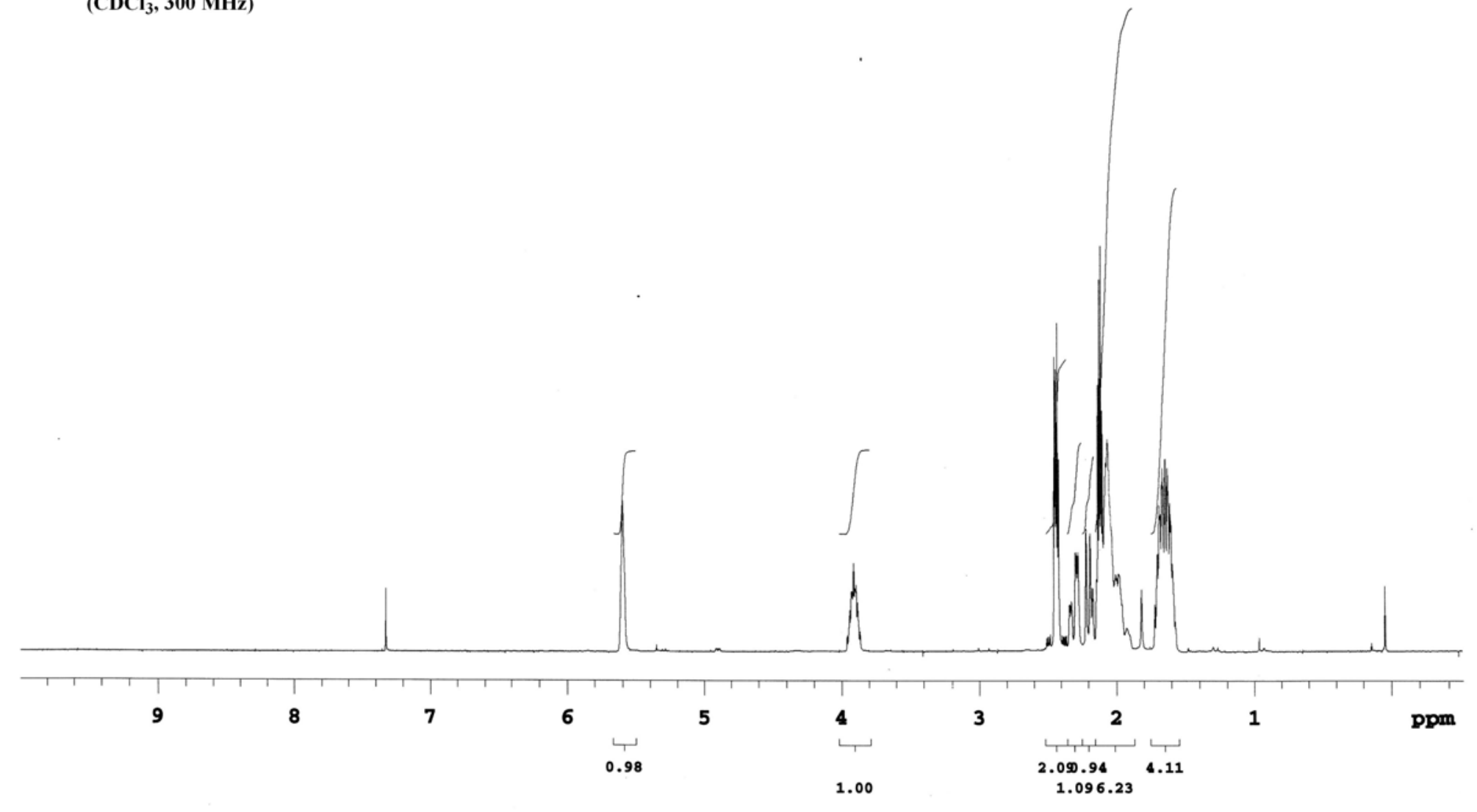


(32): (+/-)-1-Cyclohex-1-en-1-ylpent-4-yn-2-ol

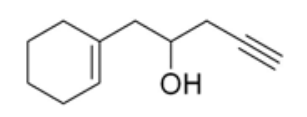

(+/-)-1-Cyclohex-1-en-1-ylpent-4-yn-2-ol

$\left(\mathrm{CDCl}_{3}, 75 \mathrm{MHz}\right)$

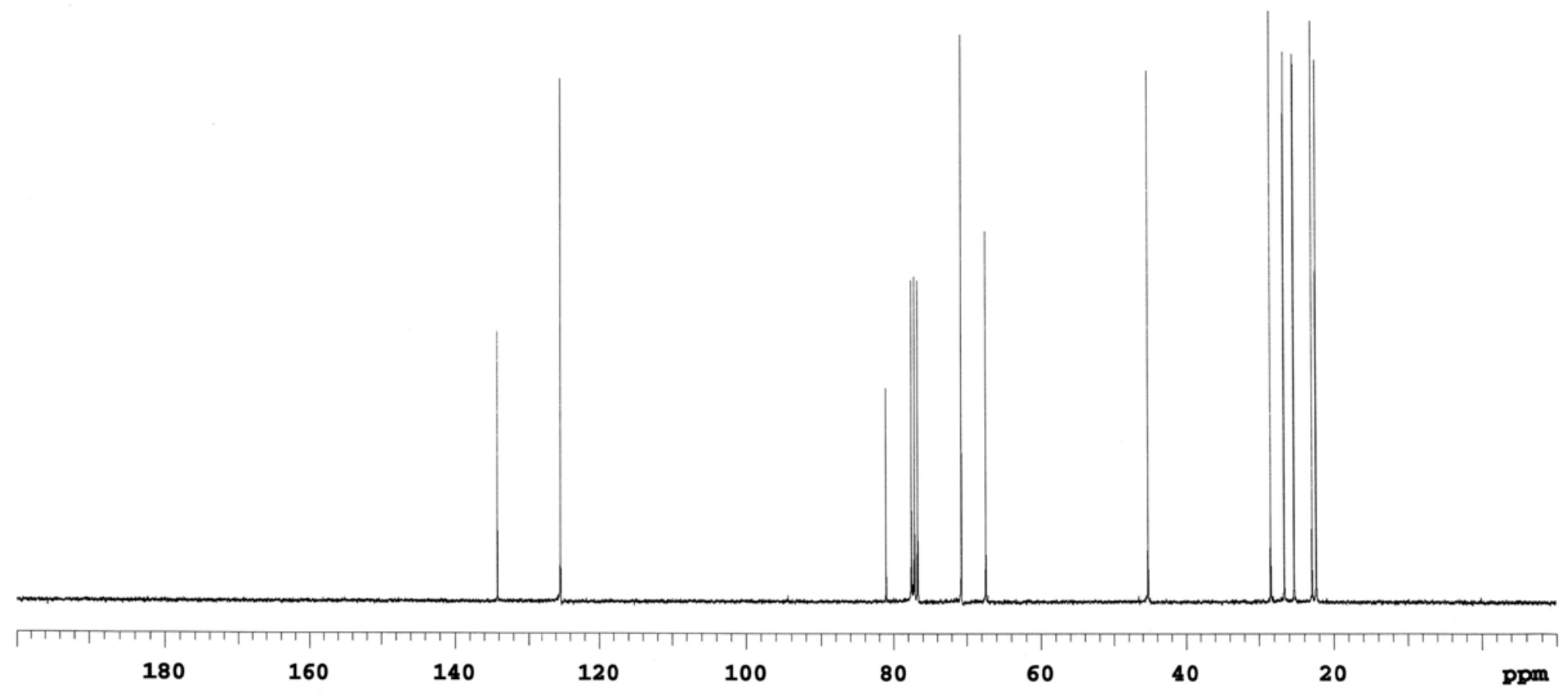


(34): (+/-)-\{[(3Z)-3-Bromo-4-phenyl-1-prop-2-ynylbut-3-enyl]oxy\}(tert-butyl) dimethylsilane

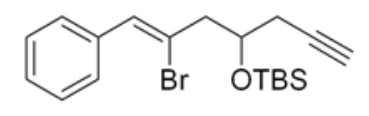

(+/-)-\{́(3Z)-3-Bromo-4-phenyl-1-prop-2-ynylbut-

3-enyl]oxy\}(tert-butyl)dimethylsilane $\left(\mathrm{CDCl}_{3}, 300 \mathrm{MHz}\right)$

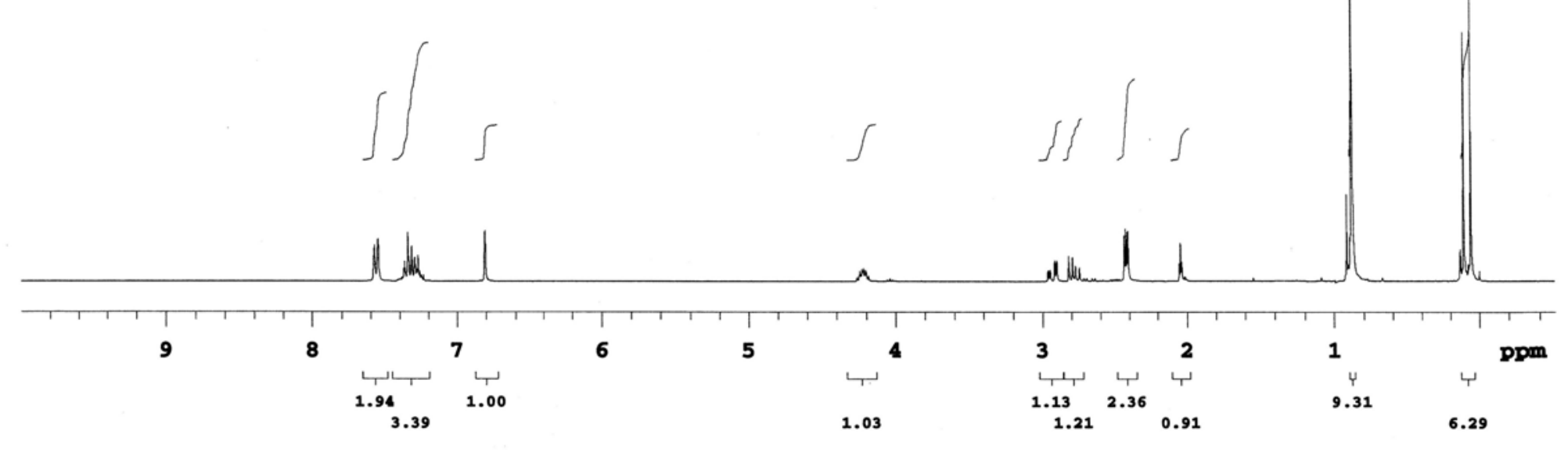


(34): (+/-)-\{[(3Z)-3-Bromo-4-phenyl-1-prop-2-ynylbut-3-enyl]oxy\}(tert-butyl) dimethylsilane

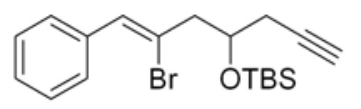

(+/-)-\{[(3Z)-3-Bromo-4-phenyl-1-prop-2-ynylbut-

3-enyl]oxy\}(tert-butyl)dimethylsilane

$$
\left(\mathrm{CDCl}_{3}, 75 \mathrm{MHz}\right)
$$

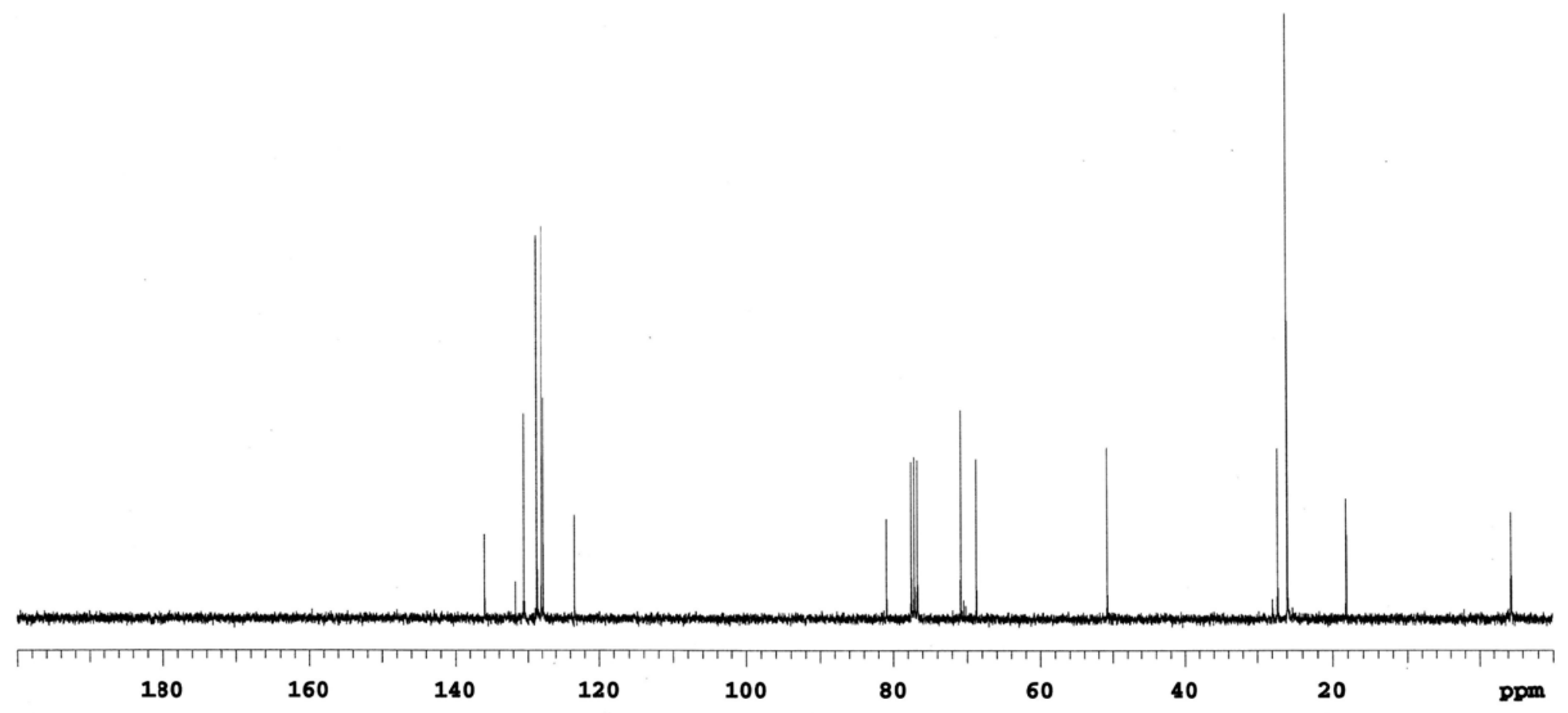


(35): (+/-)-Ethyl (2E,7Z)-7-bromo-5-\{[tert-butyl(dimethyl)silyl]oxy\}-8-phenylocta-2,7-dienoate

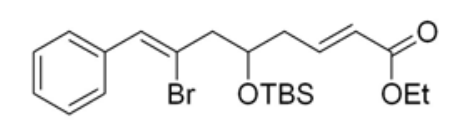

(+/-)-Ethyl $(2 E, 7 Z)-7-$ bromo-5-\{ $\{$ tert-

butyl(dimethyl)silyl] $0 x y\}-8-$ phenylocta-2,7-

$$
\begin{gathered}
\text { dienoate } \\
\left.\mathrm{CDCl}_{3}, 400 \mathrm{MHz}\right)
\end{gathered}
$$

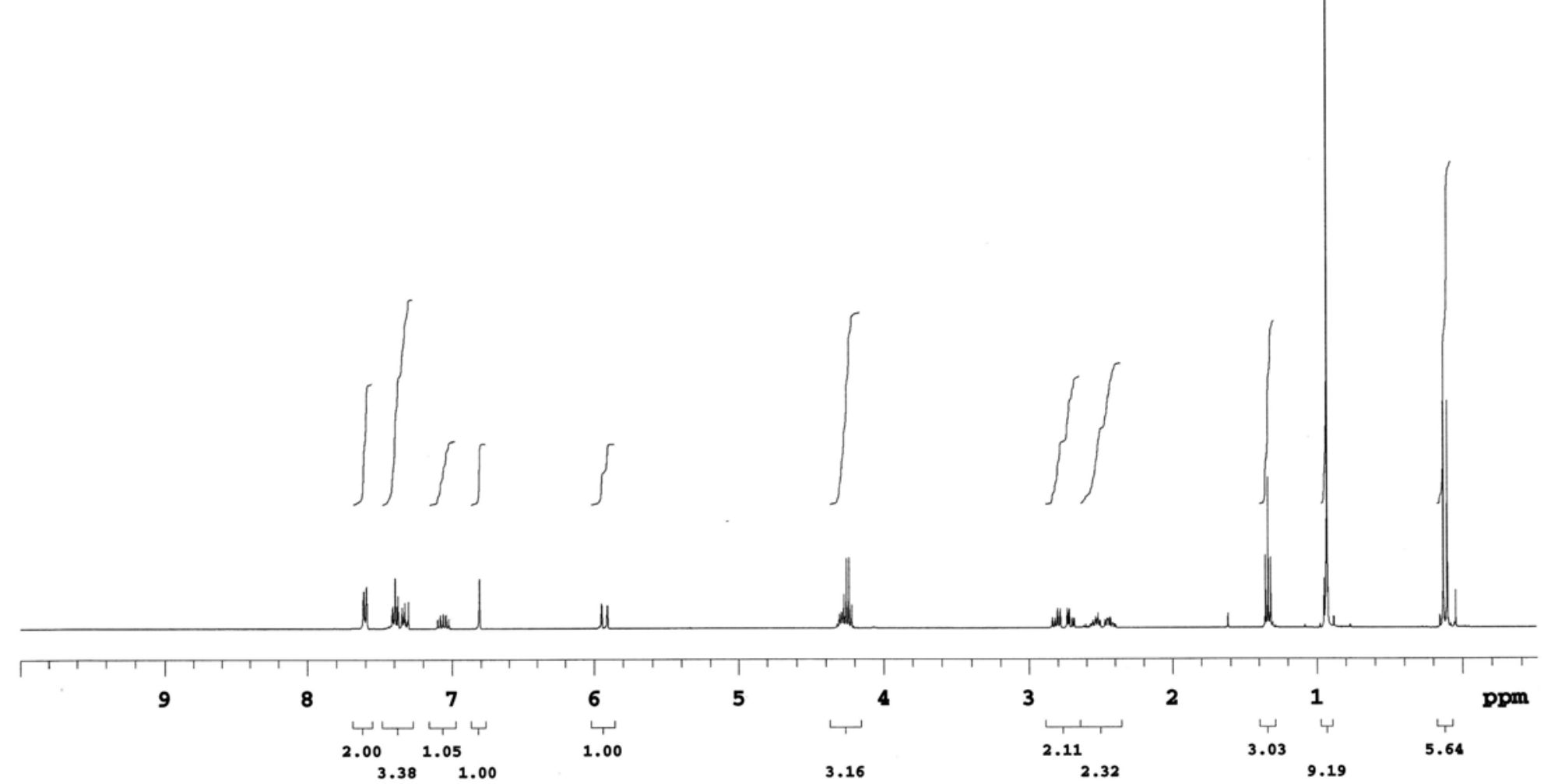


(35): (+/-)-Ethyl (2E,7Z)-7-bromo-5-\{[tert-butyl(dimethyl)silyl]oxy\}-8-phenylocta-2,7-dienoate

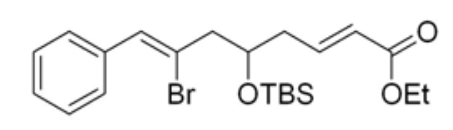

(+/-)-Ethyl $(2 E, 7 Z)-7$-bromo-5-\{ $\{$ tert-

butyl(dimethyl)silyl] oxy $\}-8$-phenylocta-2,7-

$$
\begin{gathered}
\text { dienoate } \\
\left.\mathrm{CDCl}_{3}, 100 \mathrm{MHz}\right)
\end{gathered}
$$

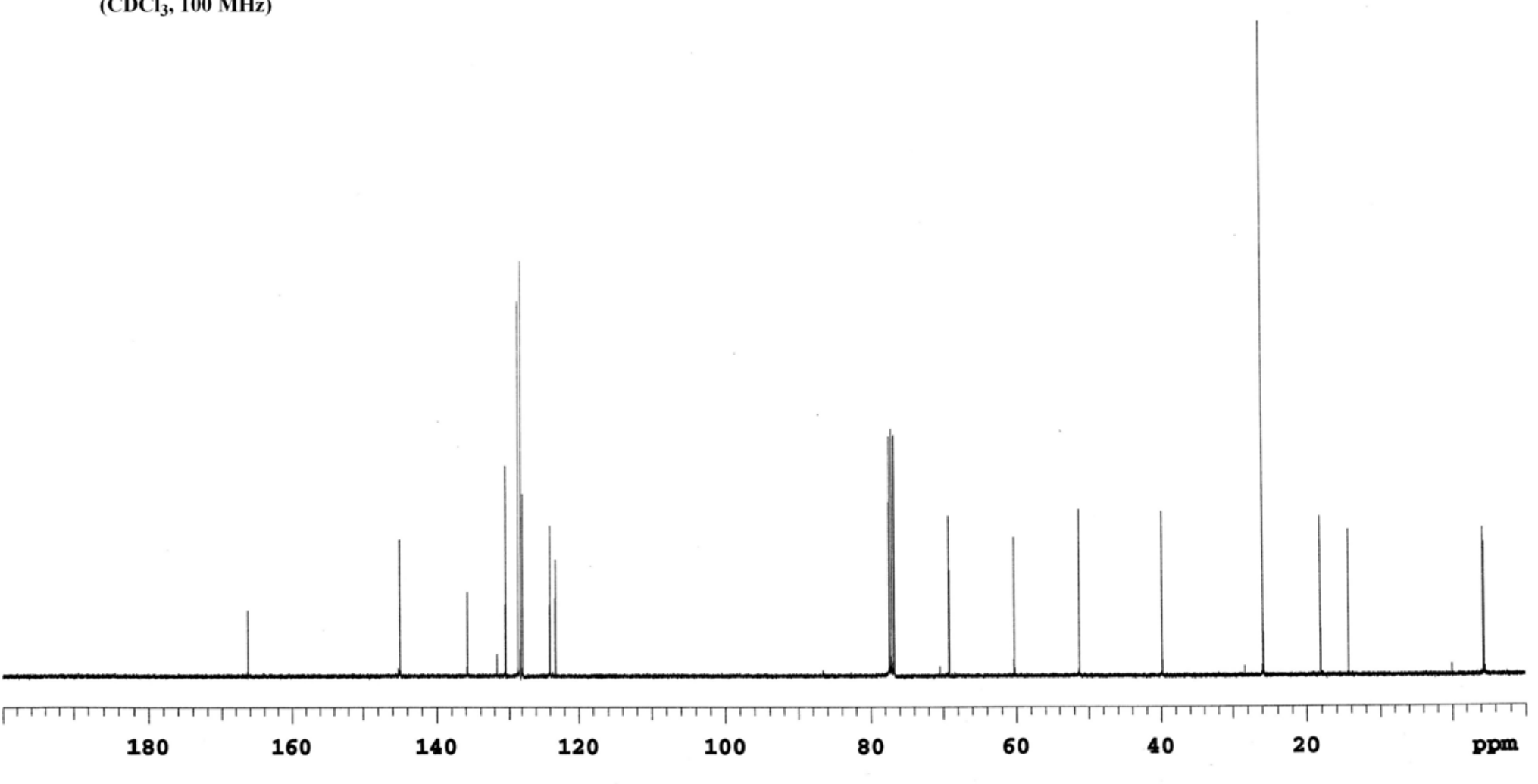


(36): (+/-)-Ethyl (2E,7Z)-7-bromo-5-\{[tert-butyl(dimethyl)silyl]oxy\}-3-methyl-8-phenylocta-2,7-dienoate

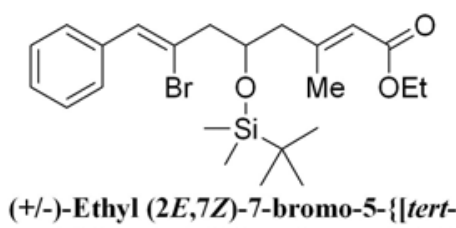

(+/-)-Ethyl (2E,7Z)-7-bromo-5-\{ltertbutyl(dimethyl)silyl] oxy\}-3-methyl-8-

phenylocta-2,7-dienoate

$\left(\mathrm{CDCl}_{3}, 300 \mathrm{MHz}\right)$

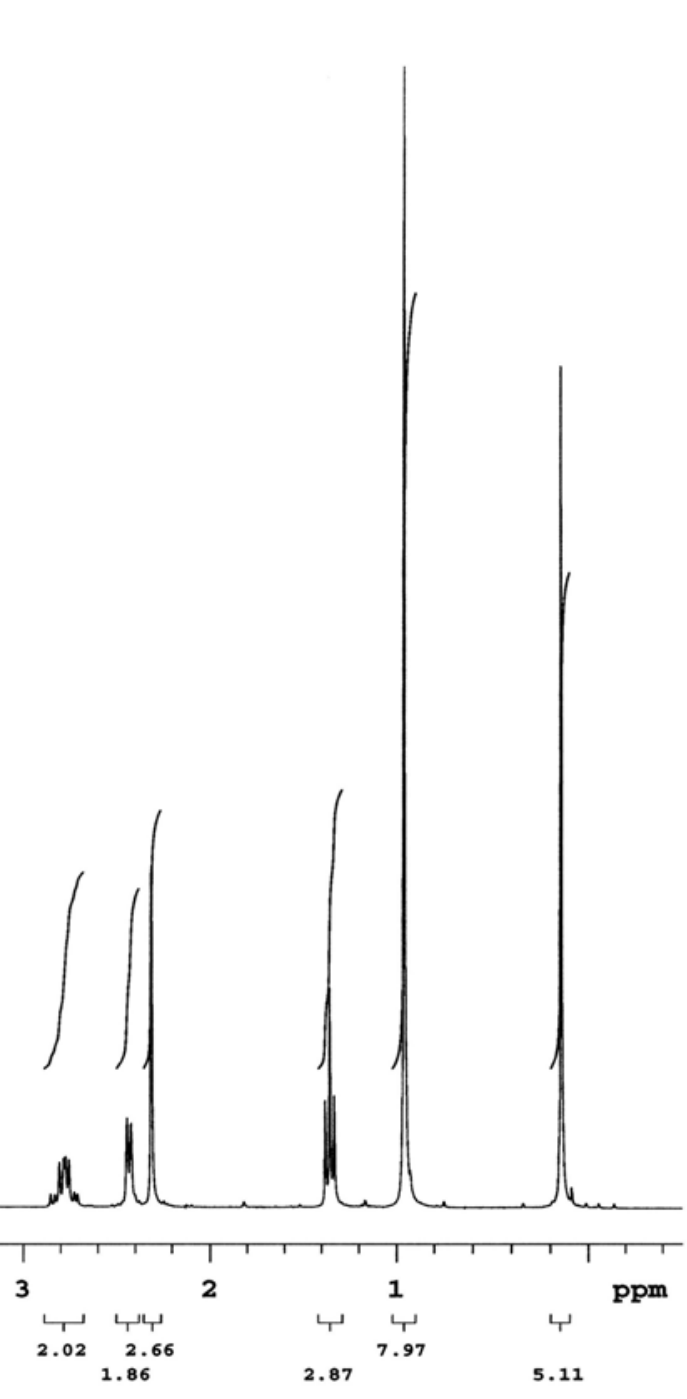


(36): (+/-)-Ethyl (2E,7Z)-7-bromo-5-\{[tert-butyl(dimethyl)silyl]oxy\}-3-methyl-8-phenylocta-2,7-dienoate

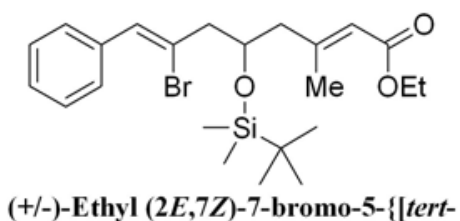

butyl(dimethyl)silylloxy\}-3-methyl-8

phenylocta-2,7-dienoate $\left(\mathrm{CDCl}_{3}, 75 \mathrm{MHz}\right)$

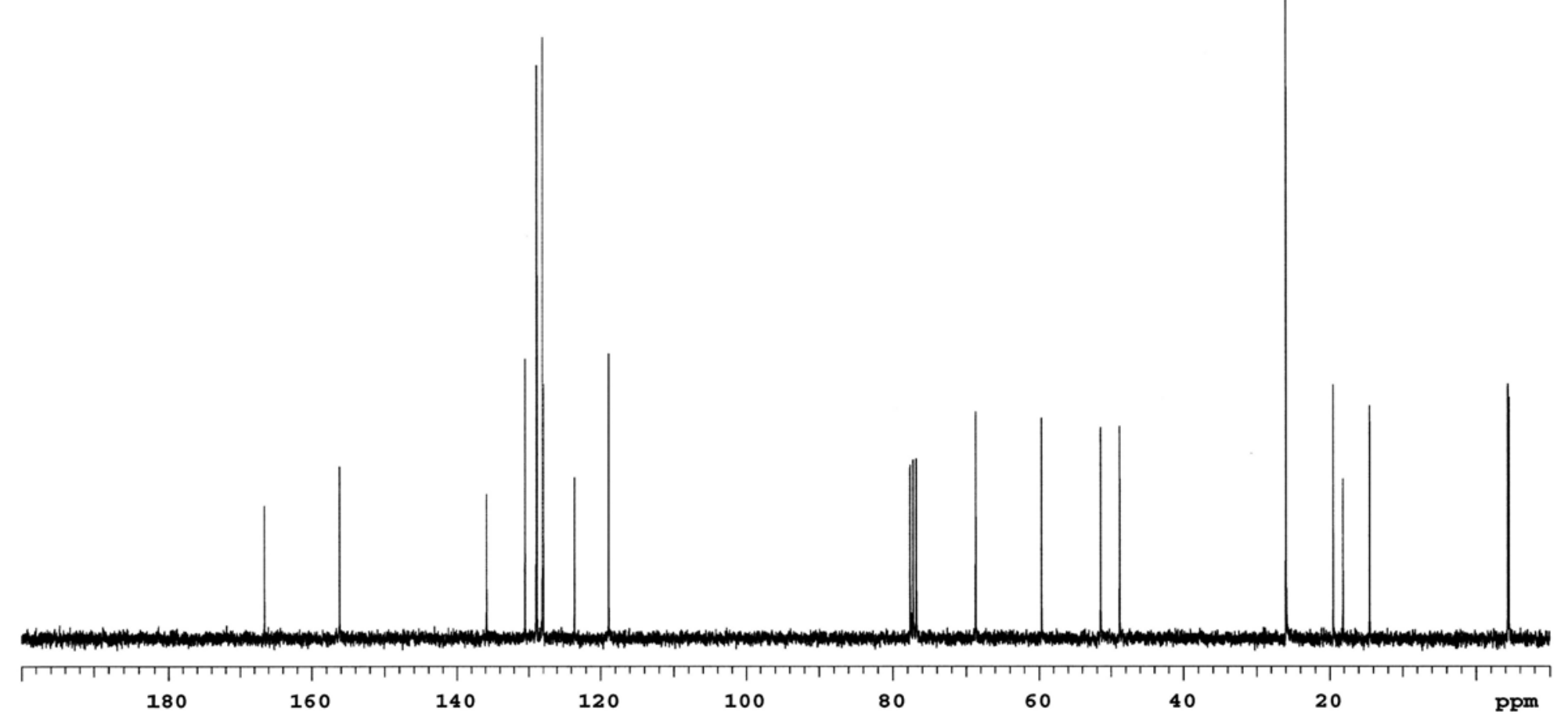


(37): (+/-)-Ethyl (2Z,7Z)-7-bromo-5-\{[tert-butyl(dimethyl)silyl]oxy\}-3-chloro-8-phenylocta-2,7-dienoate

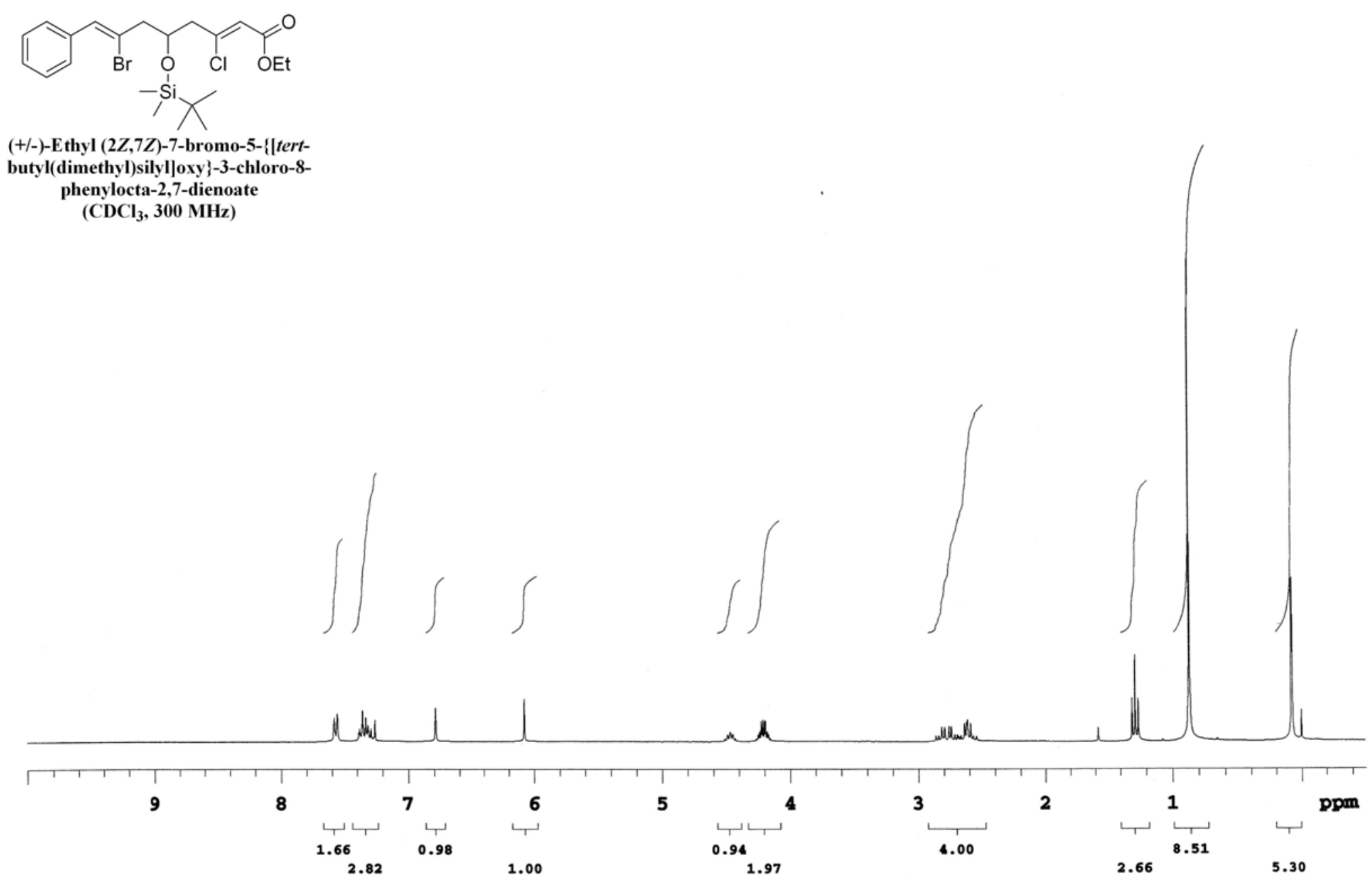


(37): (+/-)-Ethyl (2Z,7Z)-7-bromo-5-\{[tert-butyl(dimethyl)silyl]oxy\}-3-chloro-8-phenylocta-2,7-dienoate

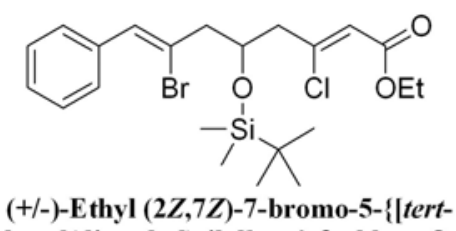

(+/-)-Ethyl (2Z,7Z)-7-bromo-5-\{ tert-
butyl(dimethyl)silyl]oxy\}-3-chloro-8

phenylocta-2,7-dienoate

$$
\text { (CDCl } 3,75 \mathrm{MHz})
$$

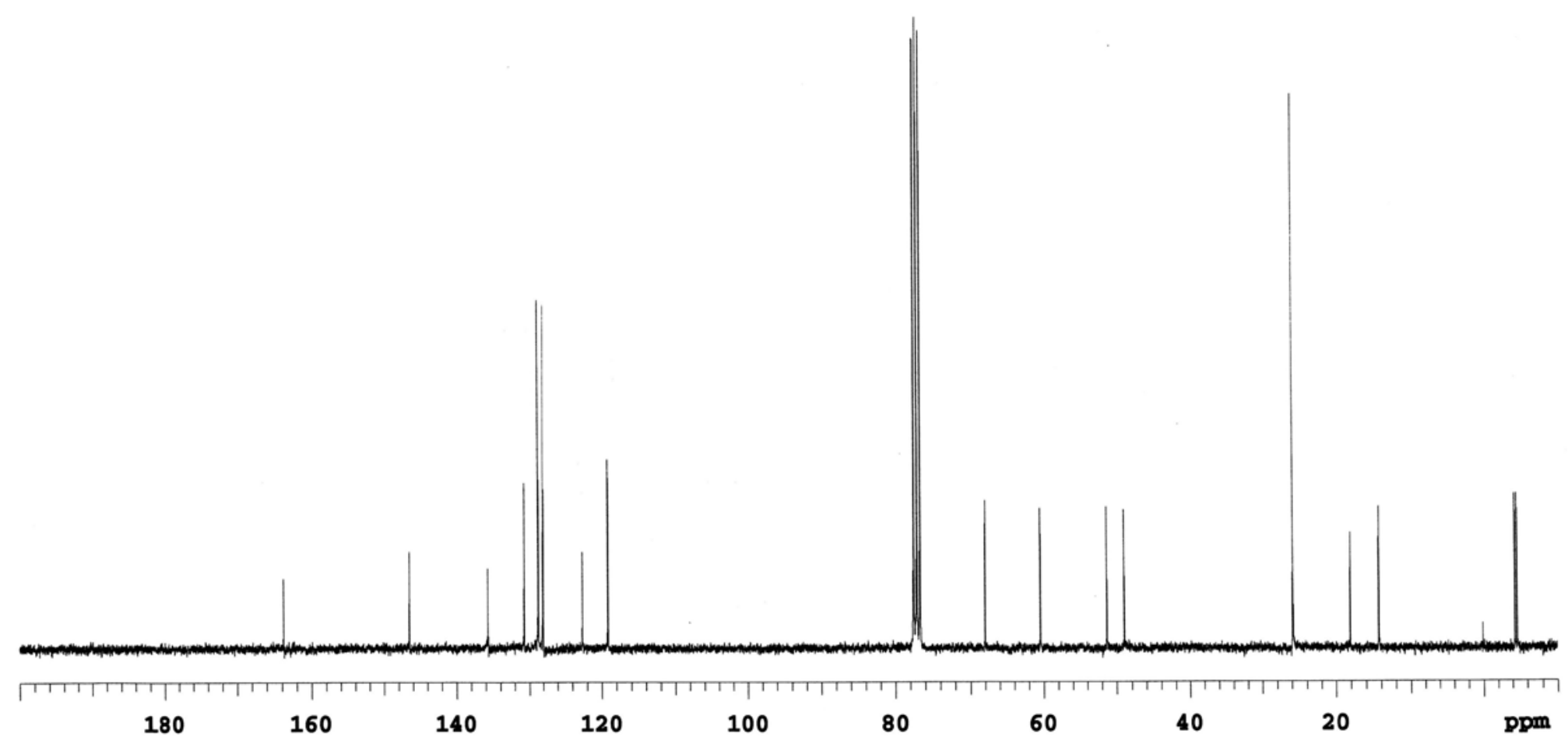

